SLAC-J-ICFA-24

\title{
HOME PAGE
}

Spring 2002

SLAC-R-655

http://www.slac.stanford.edu/pubs/icfa/

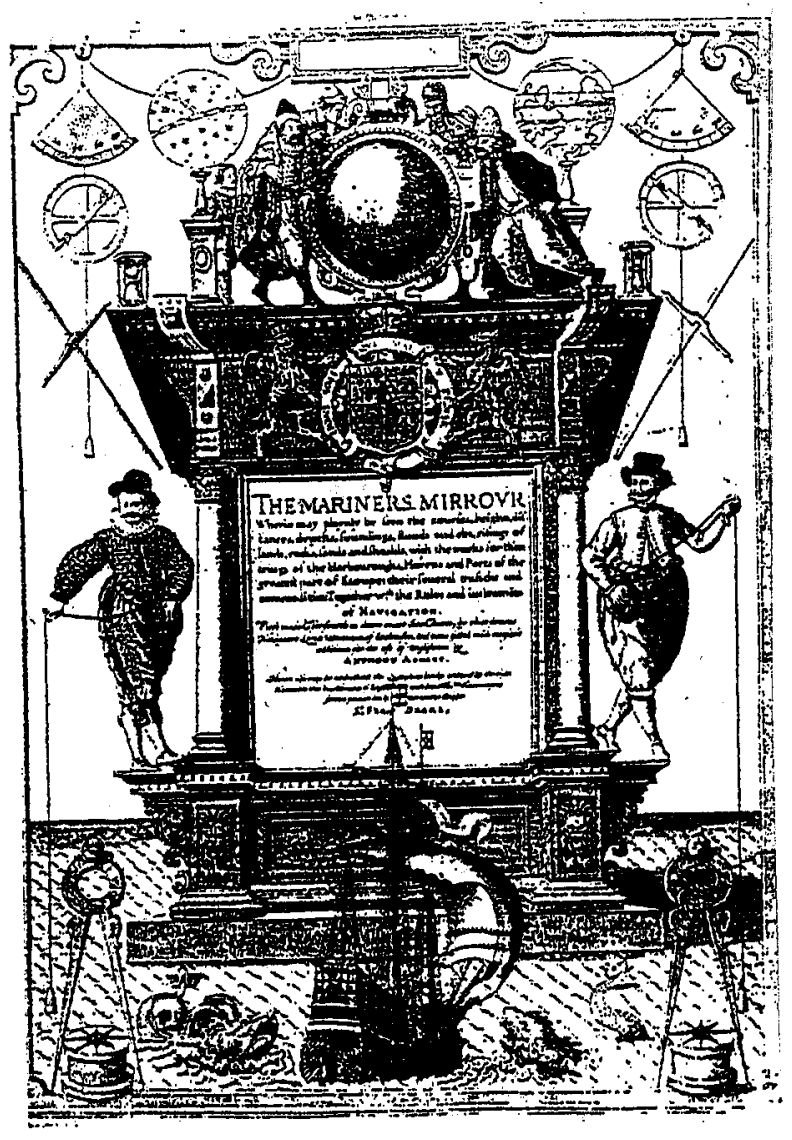

ICFA INSTRUMENTATION BULLETIN*

The publication of the ICFA Instrumentation Bulletin is an activity of the Panel on Future Innovation and Development of ICFA (InternationalCommittee for Future Accelerators).

\section{Volume 24}

\section{- Spring 2002 Issue}

* Supported by the Department of Energy, contract DE-AC03-76SF005 15. 


\section{ICFA INSTRUMENTATION BULLETIN}

The publication of the ICFA Instrumentation Bulletin is an activity of the Panel on Future Innovation and Development of ICFA (International Committee for Future Accelerators). The Bulletin reports on research and progress in the field of instrumentation with emphasis . on application in the field of high-energy physics. It encourages issues of generic instrumentation.

Publisher : $\quad$ StanfordLinear Accelerator Center SLACPublications Department Stanford, CA 94309, U.S.A.

Editor : $\quad$ J. Va'vra

Web Technical Advisers : $\quad$ J. Schwiening

The views expressed in this Bulletin do not necessarily represent those of the ICFA Panel or the editor. In all cases, the authors are responsible for their manuscripts. The printed version is mailed out in limited numbers to institutions on the SLAC Instrumentation mailing list. Issues of the ICFA InstrumentationBulletin are accessible electronically on our Web site:

\section{http $\sqrt{ } /$ www slac.stanford edu/pubs/icfa/}

Reprinting is permitted with proper acknowledgments.

Cover: The illustration depicts L. J. Waghenaer's marine atlas, "The Mariner's Mirror," published in 1588. Lucas Janszoon Waghenaer was born in Holland in the 1530s. He became a famous ship pilot in his time. In 1584, he published the atlas ("Spieghel der Zeevaerdt") which was greatly valued among mariners for centuries. This was not due only to the map content, but also to the detailed knowledge of navigation techniques of that time. The atlas, as it appears on our page, is the same one used for the Dutch to English translation. 


\section{Conference List}

-The 9-th Pisa Meeting on “Advanced Detectors,” La Biodola, Isola d'Elba, May 25-31,2003.

Web site: http://www.pi.infn.it/pm/2003/,

-20032 IEEE Nuclear Science Symposium and Medical Imaging Conference, Portland, Oregon, USA, October 19-25, 2003. Web site: http://www.nss-mic.org. 


\section{Table of Contents}

- J. Va'vra, "Physics and Chemistry of Aging - Early Developments"

- M. Titov, M. Hohlmann, C. Padilla, N. Tesch, "Summary and Outlook of the International Workshop on Aging Phenomena in Gaseous Detectors (DESY, Hamburg, October 2001)"

- Y. Bagaturia et al., "Studies of Aging and High Voltage Break Down Problems during Development and Operation of MSGC and GEM Detectors €orthe Inner Tracking System of HERA-B"

- M. Capeans, "Aging of Gaseous Detectors: Assembly Materials and Procedures" 


\title{
Physics and Chemistry of Aging - Early Developments+
}

\author{
J.Va'vra* \\ Stanford Linear Accelerator Center, Stanford, CA 94309, USA
}

\begin{abstract}
The aging phenomena are very complex physical and chemical processes. The author attempts to qualitatively discuss various physical processes contributing to aging. The satisfactory quantitative explanation is not presently available. In this sense, there is little progress made since the 1986 LBL Aging Workshop. However, what was accomplished during the past decade is a heightened awareness from the research and management sides to pay more attention to this problem, and as a result a number of aging tests have increased in quantity and quality. These efforts will undoubtedly yield some new results in the future. Examples in this paper are mainly from a "pre-LHC and preHERA-B era of aging," where the total charge dose is limited to much less than one $\mathrm{C} / \mathrm{cm}$.
\end{abstract}

\section{Introduction}

When I talk about aging, I refer to the general deterioration of the detectors during their operation; i.e., I do not restrict to usual wire aging which typically creates anode wire coating. The aging phenomenon is very complex. A multi-layer structure of an onion is a good model to describe it, where each particular process is on a separate layer. There are simply too many variables in the problem, and therefore, it is too naive to expect that one can express the aging rate using simpleminded variables, such as the accumulated charge per length of wire, etc. The correct variables include the cross-sections, the electron or photon energies, the electrostatic forces, the dipole moments, the chemical reactivity of atoms and molecules, etc. Presently, we do not know the relationship between the microscopic and the macroscopic variables we can control. Therefore, it is difficult to truly understand any aging measurements quantitatively from the physics point of view. At best, we can describe it qualitatively and measure some systematic dependencies. The aim of this workshop is quite modest, as we want to find a simple practical remedy for each particular process on a single layer of an onion, even if we might not understand the full problem.

Plasma chemistry is the closest branch of science which can shed some "practical" light on the detector aging phenomena. This was already realized before the 1986 LBL Aging Workshop [1], and a search was under way to correlate the aging phenomena with plasma chemistry observations (see, for example, references 2 and 3). Apart from similarity of the energy range of electrons, the operating parameters are quite different [2], and therefore it would appear that one couldn't share

\footnotetext{
${ }^{+}$Invited talk at the International Workshop on Aging Phenomena in Gaseous Detectors, DESY, Hamburg, Germany, October 2-5,2001

* Work supported by the Deparment of Energy under contract DE-AC03-76SF00515.
} 
the experiences significantly. Yet, in retrospect, some plasma chemistry conclusions proved to be directly applicable to our field, at least qualitatively. Some examples include a beneficial role of oxygen, oxygen-based molecules such as water or alcohol or DME, $\mathrm{CF}_{4}$, etc. Still, one cannot apply them religiously, because additives in some applications simply do not work, or may be even harmful. It seems to me that if plasma chemistry is to contribute directly to the high-energy physics detector field, it may have to allocate more time to operate tests under very similar conditions, i.e., at one bar of pressure instead of at a few Torr, using similar gases, etc.

\section{Important Physics Processes Involved in Aging Phenomena}

I will discuss qualitatively some of the most typical physical processes, which play an important role in the aging phenomena.

\subsection{Production of $\gamma$ 's}

Photons are responsible for the secondary electron emission processes, which can result in the positive feedback mechanisms or avalanche growth (avalanche breeding). Typical processes responsible for the photon production are:

- electron-atom collisions in the avalanches $\left(\mathrm{e}^{-}+\mathrm{A} \rightarrow \mathrm{e}^{-}+\mathrm{A}^{*} \rightarrow \mathrm{e}^{-}+\mathrm{A}+\gamma\right)$,

- positive ion-electron recombination at the cathode $\left(\mathrm{A}^{+}+\right.$Cathode $\left(\mathrm{e}^{-}\right) \rightarrow \mathrm{A}+\gamma$,

$$
\left.\mathrm{E}_{\gamma}=\mathrm{E}_{\text {ioniz.potential }}-\mathrm{E}_{\text {work function }}\right) \text {, }
$$

- positive ion-electron recombination in the avalanches $\left(e^{-}+A^{+} \rightarrow A^{*}+\gamma, E_{\gamma}=E_{e}+\left(E_{i}-E_{k}\right)\right)$.

\subsection{Secondary Electron Emission}

The secondary electron emission processes are responsible for the creation of positive feedback mechanisms. These processes are especially devastating as they can rapidly increase the total deposited charge dose in the advanced stages of aging. The secondary electrons can play a similar role as the secondary neutrons in the $\mathrm{U}^{235}$ fission. Their typical examples and characteristic signatures are (the way in which we can recognize them, for example, on an oscilloscope):

- photons, generated during the ion-electron recombination at the cathode, produce secondary electrons through photoeffect or via a photosensitive film developed on the cathode (signature: the primary pulse is followed by secondary pulses, which are delayed by a long delay due to the slow drift of positive ions);

- avalanche photons, caused for example by carbon excitations $\left(\mathrm{e}^{-}+\mathrm{C} \rightarrow \mathrm{C}^{*}+\gamma, \mathrm{E}_{\gamma} \sim 156,166\right.$, $193 \mathrm{~nm}$ ), interacting with the cathode surface (signature: the primary pulse is followed by secondary pulses with a short delay);

- avalanche photons interacting with the gas; for example, $\mathrm{CF}_{4}$ gas mixed with $\mathrm{TMAE}\left(\mathrm{e}^{-}+\mathrm{CF}_{4}\right.$ 
$\rightarrow\left(\mathrm{CF}_{3}^{+}\right)^{*}$ or $+\left(\mathrm{CF}_{4}^{+}\right)^{*} \rightarrow \gamma, \mathrm{E}_{\gamma} \sim 160 \mathrm{~nm}$, and $\left.\mathrm{E}_{\gamma}+\mathrm{TMAE} \rightarrow \mathrm{TMAE}^{+}+\mathrm{e}^{-}[4,5]\right)$

(signature: the primary pulses start growing in amplitude and time);

- emission on sharp points on the cathode (signature: a very steep gain-voltage characteristic with a steep threshold behavior),

- the Malter effect [6], which is a "classical" positive feedback established between the amplification and the secondary electron emission from the cathode (signature: the current starts as single electrons and can grow up to hundreds of nA. The effect is very localized. It can be persistent even after the source of radiation is removed, if the positive feedback is strong).

Although one can observe the secondary effects with an oscilloscope, a quantitative statistical method to recognize onset of the secondary effects is to measure the single-electron pulse height spectra using a UV lamp creating photoelectrons off the electrodes. A chamber suffering from a large rate of the cathode secondary effects will show excessive tail in such distribution [4]. To illustrate this phenomenon, I will quote an example of such behavior using $\mathrm{CF}_{4}$ gas, which is known to scintillate very efficiently hard UV photons near 160nm [5]. The single-electron pulse height spectrum in $\mathrm{CF}_{4}$ is very broad, probably because of the electron attachment during the avalanche (7$35 \mathrm{kV} / \mathrm{cm}$ at 1 bar) [7]. For example, adding TMAE to $\mathrm{CF}_{4}$ will trigger avalanche breeding, resulting in a long tail in the single-electron pulse height spectrum [4]. This is because TMAE can be easily photo-ionized by the $160 \mathrm{~nm}$ photons. To stop the secondary effects in this case, one needs to add 20-30\% of $\mathrm{iC}_{4} \mathrm{H}_{10}$. Aging in $\mathrm{CF}_{4}+\mathrm{TMAE}$ gas with a strong $\mathrm{Fe}^{55}$ source in a small diameter tube chamber can also reveal another interesting effect: the current steadily increases as a function of the charge dose as the photosensitive film builds up on the cathode surface. To return to a "normal aging behavior," where the current decays away, one needs to add $\sim 20 \%$ of $\mathrm{iC}_{4} \mathrm{H}_{10}$. However, the photosensitive film remains on the cathode and the chamber will remain photosensitive even if TMAE is removed [4].

The Malter effect, which is the most devastating of all secondary electron emission effects, needs three conditions to be established: (a) an insulator on the cathode, (b) a rate of ion buildup higher than its removal from the insulating layer, and (c) some ignition mechanism.

Examples of how to establish the insulating layer are (a) avalanche producing polymers, (b) glue on electrodes, (c) gas pollutants, (d) insulating deposits left from sparks, (e) corona on a sharp point of the cathode, (f) allowing the Malter currents to go on undetected even for a few minutes, (g) poor plating, (h) resistive oxides, (i) photosensitive molecules such as TMAE, TEA, etc., (j) poorly conducting "conducting epoxies," (k) poorly conducting carbon composite materials, (l) etching a conducting layer away from the cathode, (m) finger prints, etc.

Examples of the ignition mechanisms are (a) highly ionizing heavy ions or slow protons, (b) Xrays, (c) sparks, (d) sharp points on electrodes causing corona, (e) thin anode wires to help the 
ignition, (f) background muons aligned with the TPC electric field direction, (g) running out of gas, etc.

By the 1986 LBL Workshop [1], many of us believed that the Malter current jumps immediately to rather high magnitude (ten to hundreds of nanoamperes). However, Ref. 8 showed that it could occur as a train of single electrons by providing first images of the Malter effect ever observed using the CRID RICH detector, which had an excellent 3-D single electron imaging capability [8]. Large currents, which would trip the HV power supply never occurred (trip level was set to 300nA). The single electron pulses came in bursts every 10-15 minutes, and in the location of UV fibers, which were used for calibration. Such a long time constant would be consistent with a very high cathode film resistance of $\rho_{\mathrm{V}} \sim 2 \times 10^{15} \Omega . \mathrm{cm}$. Such a high value rarely occurs, and it may probably happen only in a very tight gas system with a very low humidity, and with a vacuum distilled TMAE, which aims to remove water from TMAE. In addition, because of charge exchange, TMAE ions are delivering a charge to cathode, and thus provide a fresh deposition of an insulating film. One should mention that prior to the very first appearance of the Malter effect, the UV fibers were producing a continuous photoelectron rate of $\sim 10 \mathrm{~Hz} / \mathrm{cm}$ of wire length for $\sim 2$ years. This was apparently enough to create an insulating film on the cathode in locations corresponding to the fiber positions only. The Malter effect was observed both on the high voltage cathode ( 1.2 $\mathrm{m}$ away), and the detector cathode (a few mm away). Fortunately, because of vigilance, the problem was diagnosed early and could be corrected simply by reducing the UV fiber rate by a factor of $\sim 2000$. In this case, reducing the supply of charge would be enough to stop it. The SLC accelerator never produced large enough background densities to ignite the effect during the physics running (perhaps, we would not be so lucky near a hadron accelerator). However, we did observe the Malter effect on one detector during the gating operation in early period (the gating wires serve as cathode when the gating pulse is on, which prevents ions from drifting into TPC volume), creating a standing current of 5-10 nA even after the beam went off. Based on that experience, we decided to switch off the gating circuit during the entire SLD operation. The most important lesson learned in this example is that the Malter current can consist of single electron pulses, it is localized, and it can go easily undetected if the detector does not have a single electron detection sensitivity. It is very important to catch it early before thick cathode deposits develop and consequently larger persistent currents are triggered causing a real damage to electrode structure.

Subsequent laboratory tests revealed that it is indeed very easy to ignite the Malter effect in any chamber that either has been or is exposed to TMAE [8], and it works equally well if one uses a high intensity UV Mercury lamp, or a $\mathrm{Fe}^{55}$ source. However, when similar tests were done with a CsI-based MWPC photodetector, it was not possible to ignite the Malter current. ${ }^{1}$ A possible explanation may lie in the fact that the test was done on a detector exposed to air for $\sim 10$ minutes. A

\footnotetext{
${ }^{1}$ During author's visit of A. Breskin group, Weizmann Institute, Israel.
} 
typical volume resistance of the CsI layer is $\rho_{\mathrm{V}} \sim 10^{7} \Omega . \mathrm{cm}$, if exposed to air for $\sim 10$ minutes, which is usually the case (just after evaporation, is $\rho_{\mathrm{v}} \sim 10^{10} \Omega . \mathrm{cm}$ ) [9]. It is believed that the volume resistance of TMAE prepared with a vacuum distillation method is much higher by at least 5 orders of magnitude. If true, it would mean that superbly prepared CsI photocathode with no contact to air may be closer to the Malter effect ignition than one exposed to air for a few minutes. One should add, however, that given a good ignition source, such as provided by HERA-B, for example, one could excite the Malter effect even in the CsI-based detectors [10].

In two following examples we show that certain materials are not conducting enough on a microscopic scale even though a simple-minded DVM test would pass. HERA-B experience with a polycarbonate foil doped with graphite (Pokalon-C) showed that it is not conducting enough from point of view of the Malter effect [11]. A chamber made of this material died in a few hours of operation at HERA-B, despite having survived long-term tests in X-ray setup. A likely explanation is that the real hadron beam produced very localized charge deposits such as ions or slow protons, which were not present in the X-ray beam. In this case, the problem was solved by coating the foil with $\sim 90 \mathrm{~nm}$ thick $\mathrm{Cu} / \mathrm{Au}$ layer. Similarly, author's experience with a $\mathrm{Fe}^{55}$ source-doped silver conducting paint applied on the jet chamber potential wires was equally negative [12]. This paint was applied to study the pulse propagation along the long wires. In a week, the Malter effect was observed. White deposits were observed on many neighboring potential wires in a perpendicular direction to wires. After careful cleaning of the deposits, the chamber was operated again. However, the Malter effect returned shortly. This experience points to a danger of propagation of the problem from one wire to next. Therefore it is important to segment the wire structure so one can switch off a given segment if necessary.

The next example shows the Malter effect caused by simply running out of gas in straw chambers. The gas was $\mathrm{CF}_{4}$-based mixture. The trip setting was rather high $(\sim 10 \mu \mathrm{A})$, and after several hours of allowing large currents, the inner copper conductor was gone, all etched away [13].

It is clear from the above examples that there is a relationship between a chance to trigger the Malter effect and the cathode film resistance. Let's consider an insulating film on cathode with resistance $\rho_{\mathrm{v}}$, relative dielectric constant $\varepsilon_{\mathrm{r}}$. Let's also consider the time domain only (neglect gain and spot along the wire variation). The time constant describing the neutralization of the positive charge is $\mathrm{RC} \sim \varepsilon_{\mathrm{r}} \varepsilon_{0} \rho_{\mathrm{v}}$. Let's assume that the charge is deposited in one spot with a meantime period of T, i.e., with a rate of $r=1 / \mathrm{T}$. To prevent the charge build up, one needs: $\mathrm{RC}<0.1 \mathrm{~T}$. Therefore, the maximum rate is: $\mathrm{r}_{\max } \sim 1 /(10 \mathrm{RC})=1 /\left(10 \varepsilon_{\mathrm{r}} \varepsilon_{0} \rho_{\mathrm{v}}\right)$. For $\varepsilon_{\mathrm{r}} \sim 4, \varepsilon_{0}=8.87 \mathrm{pF} / \mathrm{m}, \rho_{\mathrm{V}} \sim$ $2.8 \times 10^{7} \Omega . c m: r_{\max } \sim 10 \mathrm{kHz}$, and for $\rho_{\mathrm{v}} \sim 2.8 \times 10^{12} \Omega . \mathrm{cm}: \mathrm{r}_{\max } \sim 0.1 \mathrm{~Hz}$.

One may conclude this section by listing suggestions on how to prevent, or at least detect, the Malter effect:

- Run at the lowest gas gain possible $\left(<2 \times 10^{4}\right)$. 
- Pay keen attention to single electron signal activities.

- Monitor currents to a nA sensitivity.

- Segment HV as much as possible to have a chance to observe the effect in measured current.

- Have the HV trip setting as low as possible.

- Shut off voltages of the gas flow stops.

- Develop "clever" software, which can:

a) look for the single electron activity on a single wire,

b) look for any remnant activity in the chamber, when the beam is switched off,

c) switch off the HV power supply automatically, if a sign of persistent current occurs.

- Be vigilant.

In chapter 2.5 we will mention a possibility to cure the Malter effect with additives. In chapters 2.6 and 2.7 we will discuss other effects, which can alter the resistance of the electrodes.

\subsection{Molecular Dissociation}

The formation of molecular fragments is a necessary precursor for polymerization. Molecular fragments are formed by (a) electron or photon impact, or (b) by heat [14]. Electrons and photons in a typical avalanche have large enough energy to break the typical molecular bonds. Generally, $\mathrm{E}_{\text {Thermal dissociation }}<\mathrm{E}_{\text {Electron impact }}<\mathrm{E}_{\text {Ionization energy }}$, as detailed in Table 1. The avalanche is probably too short to create thermal dissociation, except, possibly, during the Malter effect, which is a very repetitive process.

The ideal goal would be to utilize the charge exchange mechanism to send only ions of such molecules towards the cathode, which do not polymerize easily. Based on the ionization energies listed in Table 1, one can conclude that (remember that the charge is carried by molecules with the lowest ionization energy in a given gas mix):

a) Water is less capable of performing the charge exchange when compared to DME or alcohol.

b) TMAE mixed with any gas will always deliver charge to a cathode and thus be subject to possible damage during the ion recombination process, which can produce hard photons (note that TMAE molecule can easily polymerize).

c) Water will deliver the charge to the cathode when mixed with $\mathrm{Ar} / \mathrm{CO}_{2}$ gas mix (note that water does not polymerize). 
Table 1: Dissociation \& ionization energies [14].

\begin{tabular}{|l|c|c|l|}
\hline ATOM & $\begin{array}{l}\text { Thermal } \\
\text { Dissociation }\end{array}$ & $\begin{array}{l}\text { Dissociation } \\
\text { by electron impact }\end{array}$ & $\begin{array}{l}\text { Ionization } \\
\text { Energy }\end{array}$ \\
\hline $\mathrm{Ar}$ & - & - & $15.8 \mathrm{eV}$ \\
\hline $\mathrm{Xe}$ & - & - & 12.1 \\
\hline $\mathrm{H} 2$ & $4.5 \mathrm{eV}$ & $8.8 \mathrm{eV}$ & 15.43 \\
\hline $\mathrm{O} 2$ & 5.1 & 8 & 12.06 \\
\hline $\mathrm{H} 2 \mathrm{O}$ & 4.83 & & 12.6 \\
\hline $\mathrm{CO} 2$ & 7.8 & & 13.77 \\
\hline $\mathrm{CH} 4$ & 4.3 & 4.5 & 12.6 \\
\hline $\mathrm{CF} 4$ & 5.35 & 5.2 & 11.5 \\
\hline C2H6 & 3.6 & & 10.57 \\
\hline Iso-C4H10 & 3.2 & 7 & 10.0 \\
\hline Methylal & 3.2 & & 10.49 \\
\hline Ethanol & 3.2 & & 10.15 \\
\hline Iso-propanol & 3.2 & & 9.98 \\
\hline DME & 3.2 & & 5.6 \\
\hline TMAE & 2.7 & & \\
\hline
\end{tabular}

\subsection{Polymerization of Molecules}

The polymerization of hydrocarbon molecules is often preceded by production of, for example, $\mathrm{CH}_{2}$ : radical, which is a typical precursor [15] for production of the polyethylene, an excellent insulator. The $\mathrm{CH}_{2}$ : radical has a large dipole moment and will stick preferentially to electrode surfaces. The $\mathrm{CH}_{2}$ : can be produced, for example, in the reaction $\mathrm{e}^{-}+\mathrm{CH}_{4} \rightarrow \mathrm{CH}_{2}:+\mathrm{H}_{2}+\mathrm{e}^{-}$. Gases, such as a mixture of argon and $\mathrm{CO}_{2}$, will not produce similar reaction unless there is hydrocarbon contamination. Therefore, there is indeed some logic to avoid hydrocarbons in high rate applications (above $\sim 0.5-1 \mathrm{C} / \mathrm{cm}$ ).

\subsection{Prevention of the Polymerization}

In some cases there are some additives preventing polymerization. For example, adding the following additives in the plasma chemistry processes tends to eliminate the $\mathrm{CH}_{2}$ : radical by forming stable, volatile products, which impedes the polymerization process [15]: $\mathrm{CH}_{2}:+\mathrm{H}_{2} \rightarrow$ $\mathrm{CH}_{4}, 2 \mathrm{CH}_{2}:+\mathrm{N}_{2} \rightarrow 2 \mathrm{HCN}+\mathrm{H}_{2}, \mathrm{CH}_{2}:+\mathrm{O}_{2} \rightarrow \mathrm{CO}_{2}+\mathrm{H}_{2}, \mathrm{CH}_{2}:+\mathrm{O}_{2} \rightarrow \mathrm{CO}+\mathrm{H}_{2} \mathrm{O}, \mathrm{CH}_{2}:+$ 
$\mathrm{H}_{2} \mathrm{O} \rightarrow \mathrm{CO}+2 \mathrm{H}_{2}, \mathrm{CH}_{2}:+\mathrm{CO}_{2} \rightarrow 2 \mathrm{CO}+\mathrm{H}_{2}, \mathrm{CH}_{2}:+\mathrm{CF}_{4} \rightarrow \mathrm{C}_{2} \mathrm{H}_{2} \mathrm{~F}_{4}$. More generally, atomic oxygen reacts with hydrocarbon radicals and the end-product of this reaction are volatile molecules such as $\mathrm{CO}, \mathrm{CO}_{2}, \mathrm{H}_{2} \mathrm{O}$ and $\mathrm{H}_{2}$, which are more stable, and can be removed by a sufficient gas flow. Furthermore, organic compounds with oxygen containing groups $-\mathrm{COOH},-\mathrm{CO}-,-\mathrm{OCO}-,-\mathrm{OH},-\mathrm{O}-$ , $-\mathrm{C}=\mathrm{O}$ are generally reluctant to form polymers in the plasma environment [16]. Examples of such molecules are shown in Table 2.

Table 2: Examples of usual additives to prevent polymerization [14].

\begin{tabular}{|l|c|c|}
\hline \multicolumn{1}{|c|}{ Additive } & Chemical formula & Dipole Moment \\
\hline Water & $\mathrm{H}-\mathrm{O}-\mathrm{H}$ & $1.85 \mathrm{D}$ \\
\hline Alcohols: & $\mathrm{R}-\mathrm{O}-\mathrm{H}$ & $\sim 1.7 \mathrm{D}$ \\
\hline a) Methanol & $\mathrm{R} \equiv \mathrm{CH}_{3}$ & $1.70 \mathrm{D}$ \\
\hline b) Ehanol & $\mathrm{R} \equiv \mathrm{CH}_{3} \mathrm{CH}_{2}$ & $1.69 \mathrm{D}$ \\
\hline c) Iso-propanol & $\mathrm{R} \equiv\left(\mathrm{CH}_{3}\right)_{2} \mathrm{CH}$ & $1.66 \mathrm{D}$ \\
\hline Methylal: & $\begin{array}{c}\mathrm{R}-\mathrm{O}-\mathrm{R}^{\prime}-\mathrm{O}-\mathrm{R} \\
\mathrm{R} \equiv \mathrm{CH}_{3}, \mathrm{R}^{\prime} \equiv \mathrm{CH}_{2}\end{array}$ & \\
\hline Ethers & $\begin{array}{c}\mathrm{R}-\mathrm{O}-\mathrm{R}^{\prime} \\
\mathrm{DME}: \mathrm{R} \equiv \mathrm{R}^{\prime} \equiv \mathrm{CH}_{3}\end{array}$ & $1.30 \mathrm{D}$ \\
\hline
\end{tabular}

The above mentioned additives help to prevent the polymerization through the following processes:

- high electronegativity of oxygen.

- molecular charge exchange (avalanche-produced positive ions, which would produce higher energy photons during the recombination process, are exchanged with ions, which have lower ionization potential and does contain oxygen in its molecule. For example, if one can charge exchange into an ion of water, there is a benefit since water does not polymerize),

- molecular dipole moment (therefore they stick to anode or cathode surfaces, and therefore, the oxygen-based molecule tends to help right where it is important),

- oxygen has the freedom to make double bonds, which are stronger and allow to make more stable volatile molecules, which can be removed by gas flow $\left(\mathrm{CO}, \mathrm{CO}_{2}\right.$, etc.), - etching of the deposits (this effect competes with the deposition rate [17]).

Since water and alcohol are typical additives, we would like to present specific arguments why we think they are helpful. First, let's discuss water: (a) water prevents the start of polymerization, when introduced at the beginning through the reaction: $\mathrm{CH}_{2}:+\mathrm{H}_{2} \mathrm{O} \rightarrow \mathrm{CO}+2 \mathrm{H}_{2}$. (b) If water is introduced after the deposits are formed, it tends to stabilize the operation and prevent the Malter effect. (c) A water molecule may perform the charge exchange with some avalanche hydrocarbon ions, which will tend to avoid their polymerization during the charge recombination at the cathode. 
(d) Water by itself does not polymerize. (e) Water will help to increase the conductivity of the insulator. (f) Its large dipole moment cools electrons with less than $\sim 1 \mathrm{eV}$ (it is actually the best "cool" gas available, if we would manage to prevent a condensation). Good examples of detectors where water works well are the SLD drift chamber $\left(25 \% \mathrm{Ar}+71 \% \mathrm{CO}_{2}+4 \% \mathrm{C}_{4} \mathrm{H}_{10}+3000 \mathrm{ppm} \mathrm{H}_{2} 0\right)$, and the BaBar drift chamber $\left(80 \% \mathrm{He}+20 \% \mathrm{C}_{4} \mathrm{H}_{10}+3500 \mathrm{ppm} \mathrm{H}_{2} 0\right)$, where $\sim 85 \%$ of the gas recirculates through a $\mathrm{O}_{2}$ palladium getter. However, water additives are best suited to metal-onlydesigns, such as the classical large wire chambers. In applications involving dielectrics operating at high electric surface gradients, such as the anode wire supports or GEM amplifying structure, large amounts of water may cause surface breakdowns [18]. It can also cause a problematic chemistry in the CsI and TMAE-based, the RPC detectors, or $\mathrm{CF}_{4}$-filled chambers (see chapter 2.7).

Second, let's discuss alcohol: (a) Alcohol molecules have large dipole moments, and therefore, they will be attached directly on the electrodes. (b) Alcohol molecules will perform the charge exchange more readily than water. (c) If alcohol is introduced once the deposits are formed, it tends to stabilize the operation and prevents the Malter effect [19]. (d) Alcohol molecule absorbs UV photons. (e) However, alcohol can be broken, at least in principle, to formaldehyde $\mathrm{CH}_{2}=\mathrm{O}$, which can polymerize. However, the rate of this polymerization is slower compared to the rate of the ordinary hydrocarbons, and therefore, the addition of alcohol can be considered as beneficial. (f) Various dissociation byproducts, such as $\mathrm{CH}_{2} \mathrm{O}_{2}, \mathrm{C}_{2} \mathrm{H}_{4} \mathrm{O}$ or $\mathrm{C}_{2} \mathrm{H}_{4} \mathrm{O}_{2}$ can react with aluminum and nickel and create oxides that are highly resistant [20]. (g) Last, but not least, alcohol is a solvent and can cause swelling and expansions of some plastic material, such as Mylar or Kapton, which may be important for straw tubes.

A. Boyarski showed that the Malter deposits in a form of whiskers could actually be removed by adding 200-1000ppm of oxygen to $80 \% \mathrm{He}+20 \% \mathrm{iC}_{4} \mathrm{H}_{10}$ gas and allowing large current [19]. It appears that the damaged chamber could be cured by this method. However, one should point out that the curing was done on artificially created whiskers, which have very high gradient; one has to repeat it with an oil film deposited on a cathode wire. If proven, this would be the first result in our field, which supports the tests previously reported by plasma chemists, and also by astronomers who clean the mirrors in the oxygen plasma [21].

Unfortunately, the oxygen additive is not going to help with the $\mathrm{Si}$ deposits, because the equivalent molecule to $\mathrm{CO}_{2}$ is $\mathrm{SiO}_{2}$, which is not volatile. One way to remove the $\mathrm{Si}$ deposits is to operate with the $\mathrm{CF}_{4}$ gas, and form gaseous $\mathrm{SiF}_{4}$ in avalanches. However, one has to be prepared to face the fact that $\mathrm{CF}_{4}$ introduces a very complicated chemistry, such as a possible formation of (a) HF when water or hydrocarbons are present [22], (b) resistive metal fluorides on nearby electrodes [23], (c) long-lived electronegative ions $\mathrm{F}^{-}$and $\mathrm{CF}_{3}^{-}$[24]. In addition, $\mathrm{CF}_{4}$ is producing hard UV photons in the avalanches $(\sim 160 \mathrm{~nm})$ [5], which may trigger the secondary electrons on nearby electrodes. A better way to deal with the Si deposits might be to remove the source of the silicone. Known sources are (a) O-rings with Si-based grease, (b) valves with Si-based grease, (c) G-10 
boards, (d) molecular sieves, (e) general pollution of the system, (e) possibly copper tubing, which may be produced with a help of Si-based grease, etc. One way to protect against the Si pollution is to use the semiconductor industry quality gas system.

Despite certain dangers involved in using $\mathrm{CF}_{4}$ gas, a temptation to use it is strong because it is a very fast gas suitable for high rate applications at future high luminosity accelerators. According to Plasma Chemistry, $\mathrm{CF}_{4}$ gas is an excellent etching additive equal to $\mathrm{O}_{2}$ [14], and therefore it should help to remove possible polymerization deposits, which can be present due to a presence of hydrocarbon impurities. A nice example of this effect is the work of Openshaw and his co-workers in Ref. 25. Several chambers were aged in $50 \% \mathrm{Ar}+50 \% \mathrm{C}_{2} \mathrm{H}_{6}$ and $50 \% \mathrm{Ar}+50 \% \mathrm{C}_{2} \mathrm{H}_{6}+0.2 \%$ Ethanol, causing a pulse height reduction of $25-30 \%$ and visible deposits on the anode wire. After switching to $80 \% \mathrm{CF}_{4}+20 \% \mathrm{C}_{4} \mathrm{H}_{10}$, the pulse height and currents recovered to greater than $98 \%$ of their initial values. The authors stress that all of the recovery tests have been done with $80 \% \mathrm{CF}_{4}+20 \% \mathrm{C}_{4} \mathrm{H}_{10}$ gas mixture and that introducing different components or even changing the relative proportions of $\mathrm{CF}_{4}$ and $\mathrm{C}_{4} \mathrm{H}_{10}$ could totally change the chemical reactions. Wise, Kadyk and Hess studied the aging properties of $\mathrm{CF}_{4} / \mathrm{C}_{4} \mathrm{H}_{10}$ gases and have found two regions of higher anode depositions, one in the region of low concentration of $\mathrm{CF}_{4}(0-20 \%)$ and one in the region of high concentration of $\mathrm{CF}_{4}(85-100 \%)$ [23]. The existence of the second region was not expected. In addition to this complexity, Kadyk reported an amazingly rapid aging rate of $\sim 123000 \% / \mathrm{C} / \mathrm{cm}$ in $50 \% \mathrm{Ar}+40 \% \mathrm{CF}_{4}+10 \% \mathrm{O}_{2}$ (with a gold-plated anode wire) [3,23], a mixture expected to be strongly etching. No wire deposit could be seen under am optical microscope. In this case, one is likely dealing with resistive metal-fluoride film deposits on the cathode (see further discussion in chapter 2.7 and Ref. 23). Gold-plated wires do not age in $\mathrm{CF}_{4}$ in this case (see chapter 2.7 for other aspects through). Because of the formation of resistive metal-fluoride films, the current drawn in accelerated aging test is not a reliable indicator of anode aging for $\mathrm{CF}_{4}$-rich gases. Instead, one should use the pulse height measurement and an analysis of deposits. I would like to add that an understanding of these details shows the difficulty of the detector aging science. Nevertheless, as I said, despite certain dangers, a gas mix $95 \% \mathrm{Ar}+5 \% \mathrm{CF}_{4}$ is a current candidate for gaseous detectors, such as Micromegas, to operate at high rate [26]. Because there is no quencher of hard UV photons in this gas, one should operate at very low gas gain, which may eliminate the single electron detection capability. In addition, the electrodes should be gold plated, and the system should have low hydrocarbon contamination.

\subsection{Electrolytic Processes in the Insulators}

By electrolytic processes we mean that current is made of ions rather than electrons, as we are used to in metals or simple resistors. This subject was not discussed during the 1986 LBL Aging Workshop, and not much even during the DESY workshop. It is a new subject in the detector physics, and it is becoming more and more relevant mainly because of high luminosity operations, 
which are being planned. With the introduction of RPC, CsI-based and micro-strip detectors, questions can be asked what role the ionic currents play in the aging effects. This is a very complicated question, because the chemistry of ionic currents in glass, Bakelite (PhenolFormaldehyde polymer), Linseed oil and other materials is not well understood, especially from the point of view of changes in the volume resistance as a function of the accumulated charge. These materials are not simple resistors as one could naively assume. Because of a relative novelty of this type of aging, I will spend a bit more time on this topic.

(a)

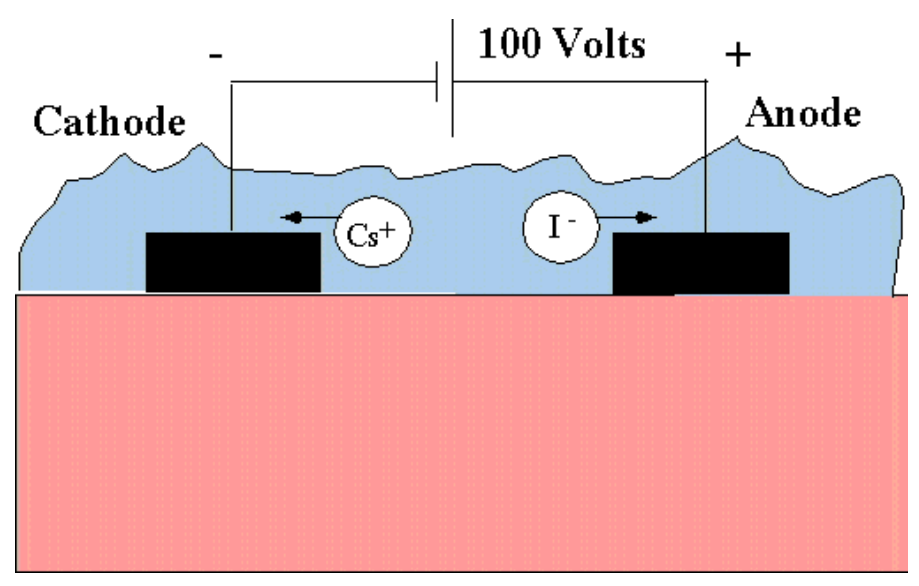

(b)

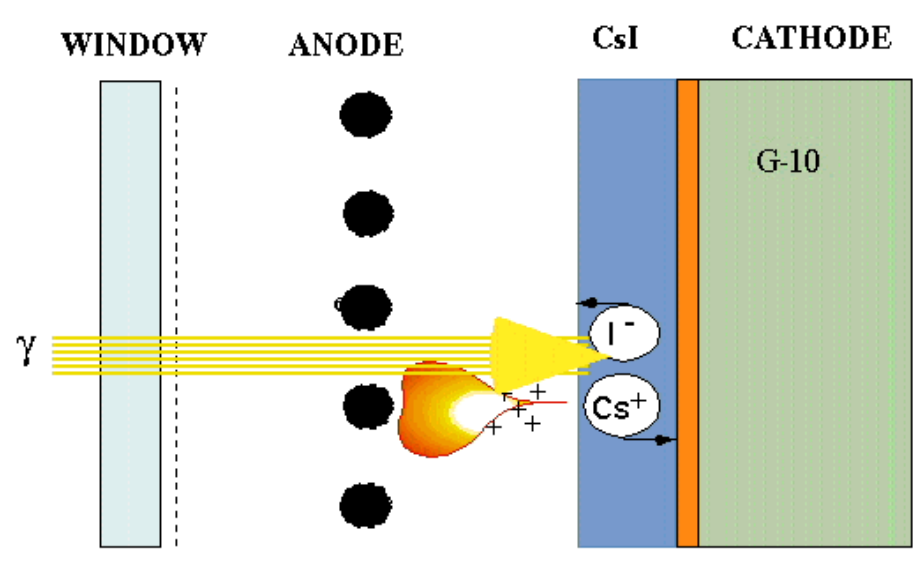

Fig.1. (a) Electrolytic current in CsI on a Micro-strip detector surface under influence of external voltage. Iodine ions move to anode, cesium ions to cathode [9]. This process will alter the resistance distribution in the CsI layer. (b) A similar electrolytic current is expected in the operating MWPC chamber under an influence of the gain and the photon flux. 
(a)

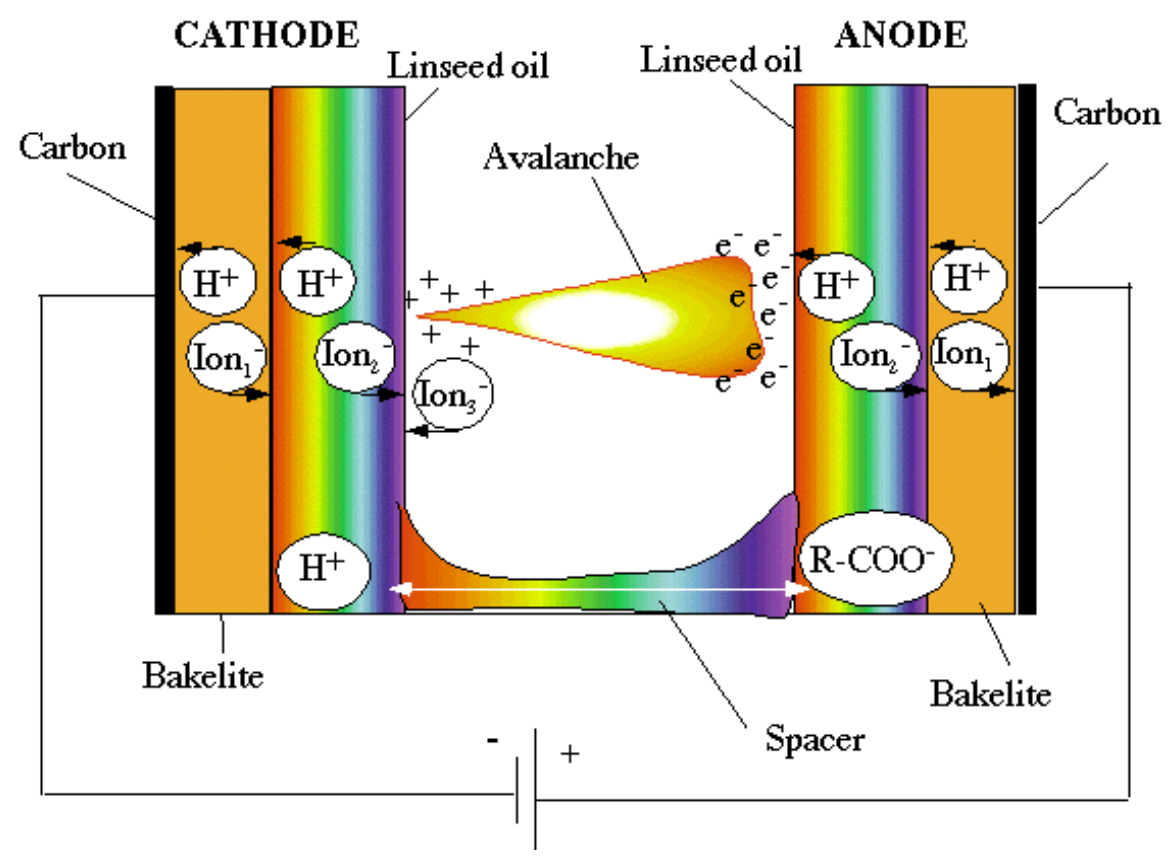

(b)

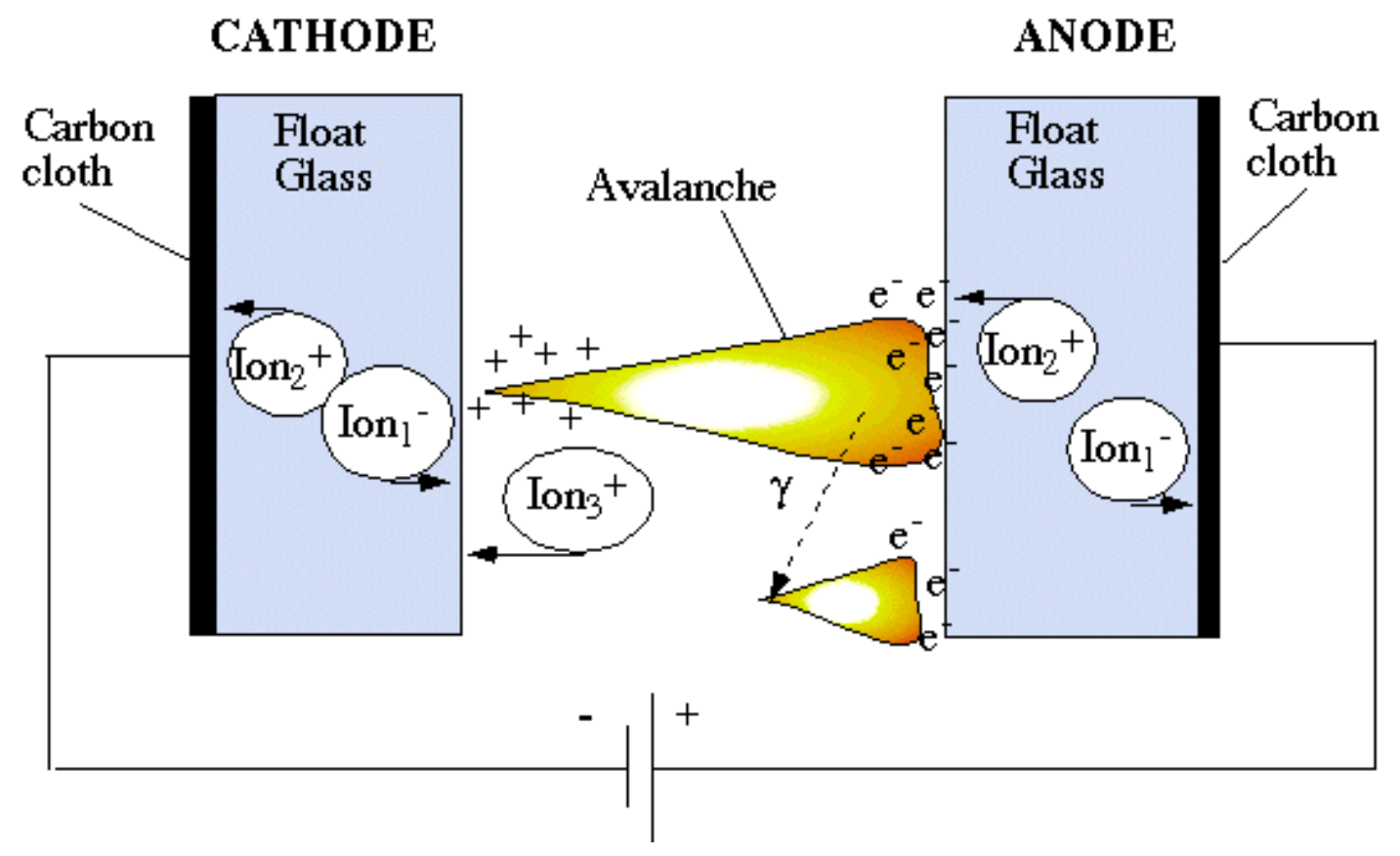

Fig. 2. (a) Ionic current in a Linseed oil-filled Bakelite RPC requires the charge exchange among three different ions of the gas, the Linseed oil and the Bakelite. (b) Although the glass-based RPC seems to be more simple from the ionic current point of view, a long term behavior of the float glass resistance is poorly understood at present. 
(a)

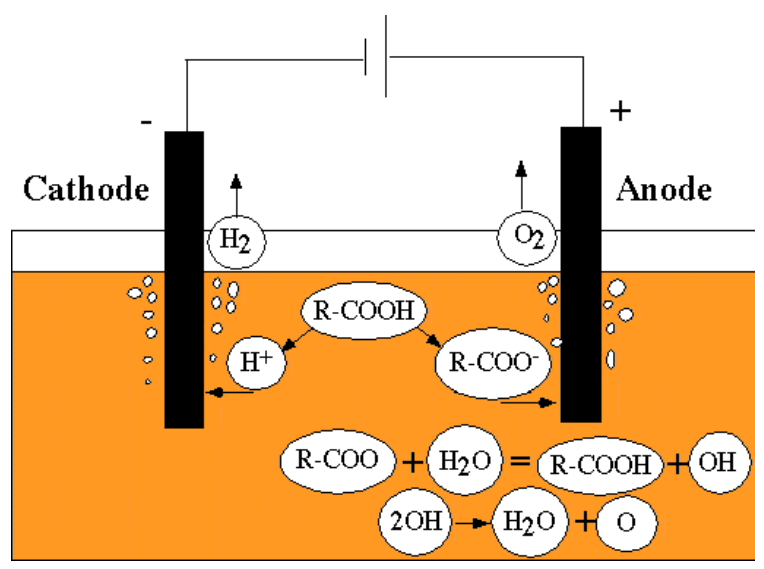

(b)

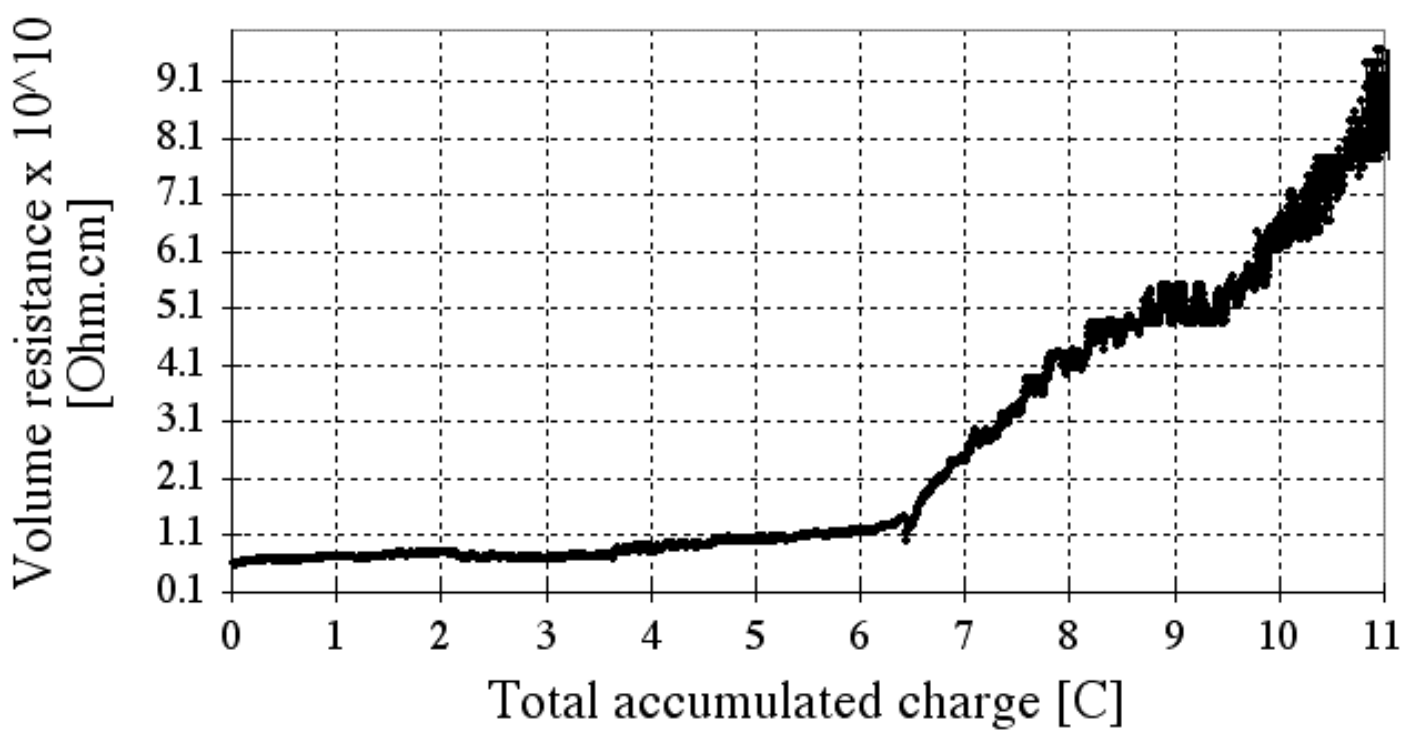

Fig. 3. (a) Proposed equivalent model of the electrolytic process in the Linseed oil [30]. (b) Volume resistance of the fresh new liquid Linseed oil as a function of accumulated charge density. The electrodes were $\sim 1 \mathrm{~mm}$ apart and a potential between them is at $3 \mathrm{kV}$. The current is not much sensitive to a voltage reversal; however, it is very sensitive to day-to-night humidity variation and to water addition. After $\sim 11$ Coulombs, the viscosity of the Linseed oil became like thick honey, full of surface wrinkles.

The current through evaporated layer of CsI is carried by the $\mathrm{Cs}^{+}$and $\mathrm{I}^{-}$ions [9]. In fact, this phenomenon can be observed visually if one arranges a simple test according to Fig.1a. Fig. 1b shows a MWPC chamber with a CsI layer on its cathode. When exposed to a gain and a high photon flux, a photocurrent through the CsI layer brings iodine ions to the cathode surface and the cesium ions in contact with the pad electrodes, where they might react chemically. The iodine on the 
surface alters its quantum efficiency and the resistance, because it is very resistive $\left(\rho \sim 1.3 \times 10^{9}\right.$ $\Omega . \mathrm{cm})$. On the other hand, Cesium is very conductive $\rho \sim 2 \times 10^{-5} \Omega . \mathrm{cm}$. As we mentioned above, a typical volume resistance of the CsI layer is $\rho \sim 10^{7} \Omega . \mathrm{cm}$, if exposed to air for $\sim 10$ minutes, which is usually the case (just after evaporation, it is $\rho \sim 10^{10} \Omega . \mathrm{cm}$ ) [9]. The iodine migration to the top of the surface is a possible mechanism to increase the resistance of the CsI layer, and therefore increase a chance of the Malter effect ignition.

Changes in conductivity of the ordinary glass were observed in the Micro-strip detectors, which prevented a successful operation, and forced the designers to choose a special expensive electron conducting glass for the substrate [27]. The conductivity of the standard glass is due to the movement of the alkaline ions. However, during the long-term operation, the alkali ions, such as sodium, migrate towards the cathode by a force of the electric field and leave a depleted layer close to anode. This leads to a permanent increase of the surface resistance. The ionic glasses therefore suffer from long-term instability. It is therefore expected that the Belle RPCs will suffer from the same phenomenon, although at this point they did not reach this particular limit yet. To use the electron conducting glasses on such a large scale, as the typical RPC size requires, is out of the question. It is interesting to point out that the BaBar/DIRC photomultiplier glass started to corrode in the presence of ultra-pure water [28]. Such water, hungry for ions, acted as a "vacuum pump" removing the sodium from the glass, which resulted in subsequent glass corrosion. Luckily for the DIRC group, the corrosion rates are slow and the detector is expected to operate for up to ten years.

The BaBar RPCs are using Bakelite electrodes covered with the Linseed oil. Again, the current is due to ionic motion, which may be even more complicated than in the glass, because both the Linseed oil and the Bakelite are more complex substances. Ionic current in such RPC requires a delicate charge exchange among different ions of the gas, the Linseed oil and the Bakelite - see Fig.2a. A model of the Belle glass-based RPC ionic current is shown on Fig.2b. The Linseed oil, made from pressed seeds, is a very complex organic compound [29]. We will simplify the complexity involved by proposing the following simple model - see Fig. 3a. One could safely assume that the initial "cocktail of molecules" also contains water. The water can also get into the Linseed oil layer either from the gas or from the Bakelite, which is known to have affinity to water. Pure water does not conduct; however, when mixed with acid, it does conduct very well through the ionic carriers. A molecule, which may facilitate current conductivity in the "uncured" Linseed oil, is the fatty acid R-COOH - an organic acid molecule. A possible sequence is as follows: (a) R$\mathrm{COOH}+$ potential $\rightarrow \mathrm{H}^{+}=\mathrm{R}-\mathrm{COO}^{-}$; (b) R-COO${ }^{-}$ion delivers the charge to anode and R-COO returns to the fluid; (c) $\mathrm{H}^{+}$ion delivers the charge to the cathode, where it forms an $\mathrm{H}_{2}$ molecule and escapes; (d) $\mathrm{R}-\mathrm{COO}+\mathrm{H}_{2} \mathrm{O} \rightarrow \mathrm{R}-\mathrm{COOH}+\mathrm{OH}$, which returns the fatty acid back into the cycle; (e) $2 \mathrm{OH} \rightarrow \mathrm{H}_{2} \mathrm{O}+2 \mathrm{O}$, and $2 \mathrm{O} \rightarrow \mathrm{O}_{2}$, which delivers oxygen near the anode. 
(a)

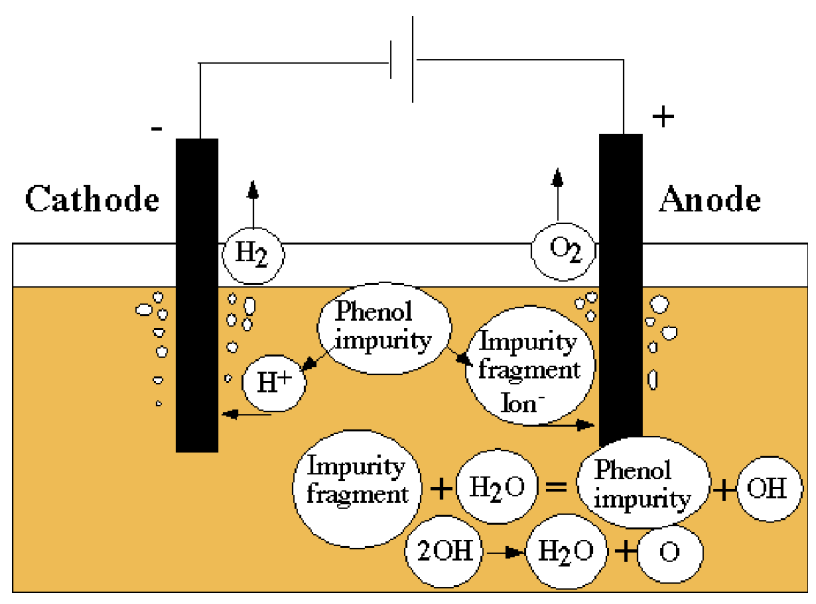

(b)

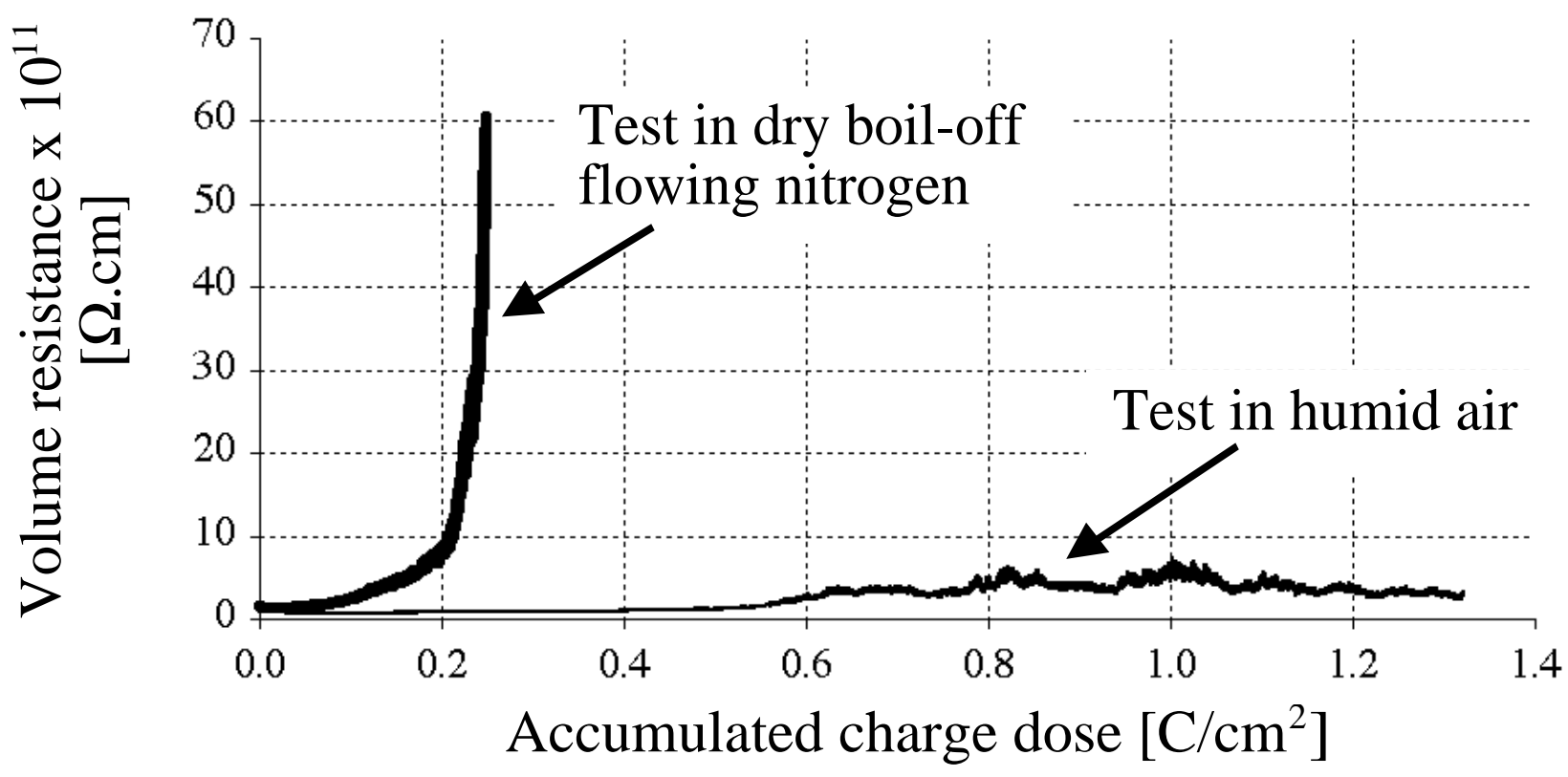


(c)

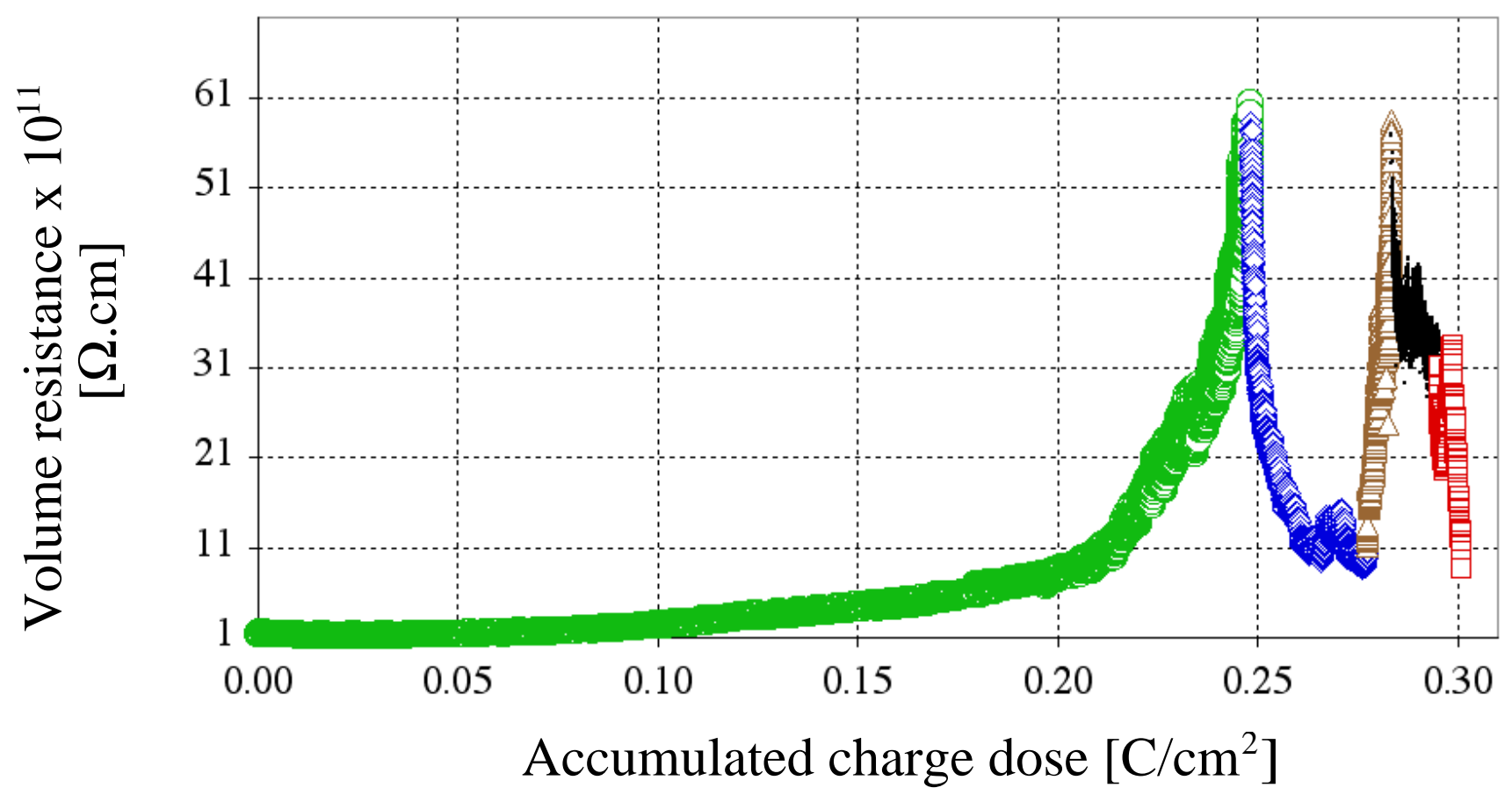

Fig. 4. (a) Proposed equivalent model of the electrolytic process in the Bakelite [30]. (b) Volume resistance of the Bakelite sheet as a function of accumulated charge density. In one case the Bakelite sheet was in air of 55$65 \%$ relative humidity variation, and in the second case a different sheet was placed in dry flowing boil-off nitrogen of less than 200-300ppm of water. (c) The Bakelite resistance can be modulated with a water in the surrounding gas: open circles (nitrogen with <200ppm of water), open diamonds (air of relative humidity of $55-65 \%$ ), open triangles (nitrogen with <200ppm of water), black dots (nitrogen with 1500-2000 of water) and open squares (air of relative humidity of $55-83 \%$ ).

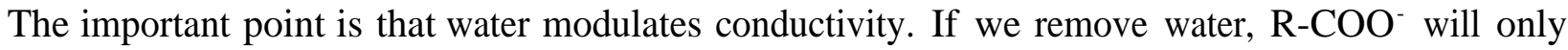
deliver the charge, R-COO will just plate on anode, but it will not return $\mathrm{R}-\mathrm{COOH}$ back into the current forming cycle; i.e., the current will slowly stop. Adding water back should restart the conduction. In fact, this was exactly what was observed in a simple bench-top electrolytic experiment that was conducted by the author [30]. Figure $3 b$ shows the author's measurement of the volume resistance increase of the fresh liquid Linseed oil. One should also point out that the liquid had some bubbles at the end, indicating a trapped gas, and after 10 Coulombs the liquid turned to a thick honey-like substance.

One can propose a similar electrolytic model for the Bakelite conductivity - see Fig. 4a. Figure $4 \mathrm{~b}$ illustrates the author's measurement showing that the Bakelite volume resistance increased by a factor of five after $\sim 0.7 \mathrm{C} / \mathrm{cm}^{2}$, if the Bakelite sheet is in humid air. However, the same resistance increase occurred after only $\sim 0.15 \mathrm{C} / \mathrm{cm}^{2}$, if it is in dry flowing boil-off nitrogen of less than $\sim 200$ 
ppm of humidity at room temperature. Figure 4c indicates that one can indeed modulate the Bakelite volume resistance by varying the humidity of surrounding gas. This is consistent with the electrolytic model. The dependency in air appears to be complicated and perhaps even nonlinear with an appearance of a threshold behavior. In principle, it is possible to have a threshold in the resistance increase if ions, responsible for the conductivity, are all used up. In fact, if several types of ions are involved, one could have several thresholds. A value between $\sim 0.15$ and $\sim 0.7 \mathrm{C} / \mathrm{cm}^{2}$ is a very high accumulated charge density, which will be relevant only at very high rate applications, or localized breakdowns. In addition, there is a dependency on temperature. For example, one week at $40^{\circ} \mathrm{C}$ in air increases the Bakelite volume resistance by a factor of $\sim 3$ [30]. Combining all these effects may increase the Bakelite resistance significantly. For example, if it would increase by a factor of 50-100, it would start affecting the efficiency due to the voltage division effect, and one may also drive the RPC into a Malter effect.

However, in addition to the above general comments, the BaBar RPCs have additional very significant problems. One is related to a poor drainage of the Linseed oil during the chamber construction, which resulted in "over-oiling" of some areas [31]. High voltage operation in $\mathrm{C}_{2} \mathrm{H}_{2} \mathrm{~F}_{4}$ gas while allowing large currents at elevated temperature resulted in a chemical reaction resulting in a very low resistance of such modified Linseed oil ( $\rho_{\mathrm{v}} \sim 2 \times 10^{8} \Omega$.cm compared to a nominal value of the fresh Linseed oil: $\rho_{\mathrm{v}} \sim$ $8 \times 10^{9} \Omega . \mathrm{cm}$ ). Each button covered with such modified oil represents effectively a short [30], which affects the efficiency nearby, especially if the Bakelite electrodes are also covered by a thick Linseed oil film. Marcello Piccolo discovered that running high currents in the damaged RPCs filled with Argon gas allows a partial or full recovery of the efficiency in these regions [32]. Subsequent author's tests showed that the resistance of the fresh Linseed oil is steadily increasing as more charge passes through, which is also accompanied with an increase of the oil viscosity with subsequent full polymerization. Further tests showed that the resistance of the Linseed oil-covered buttons, which were removed from the bad RPC regions, can be increased substantially (a factor of $>100$ ) by allowing a large current through with a total charge of $\sim 0.5-1.5 \mathrm{C} /$ button, essentially independent of polarity. That is already too much for the Bakelite resistance nearby, I think. Unfortunately, while a portion of the current flowing through the Lexan buttons covered by the Linseed oil helps to increase their resistance, a portion of the current flowing through the Bakelite electrode increases its resistance, which is bad. Because the flow of current is generally non-uniform, one may end up with a non-uniform distribution of the electrode resistance.

Many of these difficulties were supposed to be solved by a new treatment of the Linseed with a solvent to make it less viscous. However, many new problems emerged, such as sparking along the chamber edges and buttons, sparking from droplets of Linseed oil located on the cathode, etc. At a time of writing of this article, it is clear that one needs much more R\&D to understand these devices. 


\subsection{Chemistry of Gases and Nearby Electrodes}

I will choose three examples. The first example shows a rapid aging in $\mathrm{CF}_{4}$ gas after a charge dose of a few $\mathrm{mC} / \mathrm{cm}$ [23]. These results were obtained with a $99.999 \%$ pure $\mathrm{CF}_{4}$ gas, with or without Nanochem filter. Addition of $20 \%$ of $\mathrm{iC}_{4} \mathrm{H}_{10}$ stopped the rapid aging rate. This effect was explained by a formation of the resistive metal-fluoride film on the non-gold plated surfaces near avalanche (either anode or cathode, or both). The formation of such resistive layers on the cathode may then easily trigger the subsequent Malter effect, although, the resistance of such film is presently not known. It may be important to gold-plate electrode surfaces of the MICROMEGAS or GEM detectors, if $\mathrm{CF}_{4}$ gas is considered.

The second example is from the Belle experiment at KEK, Japan. Their RPC detector electrodes are made of ordinary float glass. After a successful start at full efficiency, the RPCs started to deteriorate rapidly. A massive $\mathrm{R} \& \mathrm{D}$ effort was initiated, which resulted in formulating the following model [33]. Freon gas $\left(\mathrm{C}_{2} \mathrm{H}_{2} \mathrm{~F}_{4}\right)$ together with water, in the presence of plasma formed $\mathrm{HF}$ acid which etched the glass surface. This in turn increased the current and the detection efficiency drop. It was found that initially the chambers operated with a large volume of water in gas ( 2000 ppm). The water permeated through the Polyflow gas tubing. The solution to suppress the formation of HF was to reduce water fraction from $2000 \mathrm{ppm}$ to $<10 \mathrm{ppm}$, by installing copper tubing (it may still form using hydrogen from the hydrocarbon, but this rate seems to be acceptable).

The third example is a swelling of anode [34], observed in straw tube tests operating with $70 \% \mathrm{Xe}+10 \% \mathrm{CO}_{2}+20 \% \mathrm{CF}_{4}$. A wire diameter increased by $20 \%$ after an accumulated charge of $\sim 9$ $\mathrm{C} / \mathrm{cm}$. A very complex chemistry model, involving reaction products of tungsten, oxygen and fluorine, was proposed to explain the phenomenon. Water may have played also a part to form HF molecule.

\subsection{Gas system consideration}

I will chose only one example. The measurement by Kothaus showing that even a temporary replacement of stainless steel tubing with a PVC tubing triggers a high rate of aging, and that the deterioration continues even when the original stainless steel tubing is restored [35]. It is interesting to point out the PVC tubing contains molecular chains involving $\mathrm{CH}_{2}$ molecular fragment [2]. The measurement of Kothaus is now accepted as a classical example showing the importance of choosing a high quality gas tubing. Yet, it appears that people continue to select "smelly" valves and low-quality plumbing materials (for example, a copper, tubing which was not even cleaned in hydrogen oven). Similarly, they use Si-based molecular sieves without adequate mechanical filters preventing $\mathrm{Si}$ to get into the active areas of the detector, detectors full of G-10, etc. The usual argument of the management is the cost of the gas system, of course. However, usually, the cost of a possible detector replacement is not included in such discussions. The semiconductor industry did not learn about their plumbing specification by accident. It is an expensive endeavor even for 
them; however, alternatives are even more expensive. This point was already argued at the 1986 LBL aging workshop.

\subsection{Micro-pattern detectors}

There are two major sources of degradation of the Microstrip detectors: substrate charging and deposition of polymers. It has been demonstrated [36] that the polymerization can be avoided up to at least $\sim 120 \mathrm{mC} / \mathrm{cm}$ of collected charge, which is equivalent to ten years of operation at LHC. Authors also show that one should avoid using Borosilicate glass to prevent long-term modification of gain due to charging caused by the sodium migration. Use of electron-conducting glass solves the problem (see also Ref. 27).

Based on tests with the $8.9 \mathrm{keV}$ X-rays for the Compass experiment, the GEM detectors are doing well; i.e., no loss of energy resolution or gain was reported up to a charge dose of $\sim 7 \mathrm{mC} / \mathrm{mm}^{2}$ [37].

Tests with MICROMEGAS detector are also in progress. So far, no gain loss was reported up to a charge dose of 2-3 mC/mm ${ }^{2}$ with $\mathrm{Ar}+5 \% \mathrm{CF}_{4}$ or $\mathrm{Ne}+10 \% \mathrm{C}_{2} \mathrm{H}_{6}+11 \% \mathrm{CF}_{4}$ gases [26].

\section{Conclusions}

The most devastating of all aging effects are the secondary electron emission processes, such as the Malter effect. These effects can be very localized, and can proceed undetected because the small currents are involved, at least in the early stages before a thick film develops on the cathode. The detection of such processes is difficult because the single electron sensitivity is required, often in a presence of large background. Clever software approaches may be needed to catch such effects early enough before they do damage. More frequent use of oscilloscopes would help. To clean the insulating deposits periodically either in oxygen or $\mathrm{CF}_{4}$ plasma needs more studies to dare to apply it in large systems.

Although the $\mathrm{CF}_{4}$-based gases are promising from several point of views, they may have complicated chemistry resulting in possible severe electrode corrosion. Before any large experiment decides to use such gas we recommend extensive long-term testing. For example, such gases cannot use water additive or be mixed with hydrocarbon molecules because of a formation of HF, and all electrodes should be gold-plated to prevent a formation of resistive metal fluorides.

Detectors operating at high luminosity, which use insulators, may face a new domain of aging: changes in resistance of the electrodes and supporting structure due to ionic currents. This subject is relatively new and not yet fully understood in case of the RPC detectors, although it is already recognized in the Microstrip detectors.

It is important to worry about material choice and cleanliness of the system. We do not understand the precise role of impurities and their relationship to a particular gas mix. In some cases they matter; in some other cases they do not seem to. One probably should generate a well- 
equipped centralized facility employing a dedicated pair of chemist and physicist, both serving the entire community, which would support it financially and intellectually. Perhaps, after 5-10 years, repeating systematically all significant aging tests reported in literature up to this point, under conditions which are better understood, may yield some understanding. To duplicate such facility in many places would be a mistake.

The aging science is still in its infancy, if we insist on quantitative explanations. Many new things will be learned by the time the LHC finishes. The benefits from Plasma Chemistry would be greater if there are more tests with similar conditions as in our field.

\section{REFERENCES}

[1] Proc. Workshop on Radiation Damage to Wire Chambers, LBL, Jan.1986, LBL-21170 (J. Kadyk - editor).

[2] J. Va’vra, Nucl. Instr. \& Meth., A252(1986)547.

[3] J. Kadyk, Nucl. Instr. \& Meth., A300(1991)436.

[4] J. Va'vra, J. Kadyk, J. Wise, O. Coyle, Nucl. Instr. \& Meth., A370 (1996) 352.

[5] A. Pansky, A. Breskin, A. Buzulutskov, R. Chechik, V. Elkind, and J. Va'vra, Nucl. Instr. \& Meth., A354(1995)262.

[6] L. Malter, Phys. Rev. 50(1936).

[7] P.G. Datskos, J.G. Carter and L.G. Chrisoporou, J. Appl. Phys. 71(1992)15.

[8] J. Va'vra, Nucl. Instr. \& Meth., A367(1995)353.

[9] J. Va'vra, A. Breskin, A. Buzulutskov, R. Chechik, and E. Shefer, Nucl. Instr. \& Meth., A387(1997)154.

[10] P. Krizan, private communication.

[11] Ch. Stegmann, Nucl. Instr. \& Meth., A453(2000)153 and

M Hohlmann, Nucl. Instr. \& Meth., A461(2001)21.

[12] J. Va'vra, “The 8-wire prototype," OPAL R\&D during author's stay at CERN, 1984, unpublished.

[13] K. Lang, private communication.

[14] H. Yasuda, Plasma Polymerization, Academic Press, Inc., 1985.

H. V. Boeing, Plasma Science and Technology, Cornell University Press, 1982.

A. von Engel, Ionized gases, American Institute of Physics, AIP Press, 1965.

The Handbook of Chemistry and Physics, The Chemical Rubber Co., Cleveland, Ohio.

[15] D. W. Hess, Proc. Workshop on Radiation Damage to Wire Chambers, LBL, Jan.1986, LBL-21170.

[16] H. Yasuda, Plasma Polymerization, page 113, Academic Press, Inc., 1985.

[17] H. Yasuda, Plasma Polymerization, Academic Press, Inc., 1985, and

"New Insights into Aging Phenomena from Plasma Chemistry," DESY workshop, 2001.

[18] S. Bachman et al., CERN-EP/2000-151, 11 December 2000.

[19] A. Boyarski, DESY workshop, 2001

[20] M. Atac, Proc. Workshop on Radiation Damage to Wire Chambers, LBL, Jan.1986, LBL-21170, and IEEE Trans. Nucl. Sci. NS-34 (1) (1987)481. 
[21] R. Gillette et al., Vac. Sci. Tech., 7(1070)534.

[22] J. Schreiner and M. Titov, DESY workshop, 2001.

[23] J. Wise, LBL Ph.D Thesis, LBL-32500, UC-414, August 1992, and

J. Wise, J. Kadyk and D.W. Hess, J. Appl. Physics, 74(9)5327, and

J. Va'vra, P. Coyle, J. Kadyk and J. Wise, Nucl. Instr. \& Meth., A324(1993)113.

[24] M. Copeans et al., Nucl. Instr. \& Meth., A337(1993)122.

[25] R. Openshaw, R.S. Henderson, W. Faszer and M. Salomom, Nucl. Instr. \& Meth., A307(1991)298.

[26] Y. Giomataris, private communication.

[27] G. Cicognani, P. Convert, A. Oed, J. Pannetier, "Stability of different Microstrip plates on ionic and electronic conducting glass," Proc. of the Int. Workshop on Micro-Strip Gas Chamber, Lyon, pp. 235241, December 1995.

[28] P. Bourgeois and J. Va'vra, "Corrosion of Glass Windows of DIRC PMTs," ICFA Instrumentation Bulletin, Vol. 22, Spring 2001, http://www.slac.stanford.edu/pubs/icfa/spring01/paper3/paper3.pdf.

[29] Linseed oil contains the glycerides of linolenic, linoleic, oleic, stearic, and palmitic acids with a highdegree of unsaturation of its fatty acid radicals.

[30] J. Va'vra, http://www.slac.stanford.edu/ jjv/activity/babar rpc my summary.pdf.

[31] J.Va'vra, "Short Summary of a Session on New Aging Effects - RPC detectors," DESY workshop, 2001.

[32] M. Piccolo, private communication.

[33] D. Marlow, Vienna Instrumentation Conference, Vienna, 2001, and invited talk at this workshop.

[34] A. Krivchitch, Vienna Instrumentation Conference, Vienna, 2001.

[35] D. Kothaus, Proc. Workshop on Radiation Damage to Wire Chambers, LBL, Jan.1986, LBL-21170.

[36] R. Bouclier et al., Nucl. Instr. \& Meth., A381(1996)289.

[37] S. Kappler, DESY workshop, 2001. 


\title{
SUMMARY AND OUTLOOK OF THE INTERNATIONAL WORKSHOP ON AGING PHENOMENA IN GASEOUS DETECTORS (DESY, HAMBURG, OCTOBER, 2001)
}

\author{
M. Titov**, M. Hohlmann, C. Padilla, N. Tesch
}

\begin{abstract}
High Energy Physics experiments are currently entering a new era which requires the operation of gaseous particle detectors at unprecedented high rates and integrated particle fluxes. Full functionality of such detectors over the lifetime of an experiment in a harsh radiation environment is of prime concern to the involved experimenters. New classes of gaseous detectors such as large-scale straw-type detectors, Micro-pattern Gas Detectors and related detector types with their own specific aging effects have evolved since the first workshop on wire chamber aging was held at LBL, Berkeley in 1986. In light of these developments and as detector aging is a notoriously complex field, the goal of the workshop was to provide a forum for interested experimentalists to review the progress in understanding of aging effects and to exchange recent experiences.

A brief summary of the main results and experiences reported at the 2001 workshop is presented, with the goal of providing a systematic review of aging effects in state-ofthe-art and future gaseous detectors.
\end{abstract}

\footnotetext{
**Featured talk at the IEEE Nuclear Science Symposium and Medical Imaging Conference, November 4-10, 2001, San Diego, USA
} 


\section{ICFA Instrumentation Bulletin}

\section{Introduction}

Aging effects in proportional wire chambers, a permanent degradation of operating characteristics under sustained irradiation, has been and still remains the main limitation to their use in high-rate experiments [1]. Although the basic phenomenology of the aging process has been described in an impressive variety of experimental data, it is nevertheless difficult to understand any present aging measurement at a microscopic level and/or to extrapolate it to other operating conditions. Many chemical processes are expected to occur simultaneously in the gaseous discharges surrounding the wire, and consequently a quantitative description of aging effects, which would require as a minimum a detailed analysis of all gas-phase and gas-surface reaction products, is currently not available. There is much experimental information, well summarized in [2]-[4], which suggests that wire chamber lifetime may be extremely sensitive to the nature and purity of the gas mixture, different additives and trace contaminants, materials used in contact with the gas, geometry of electrodes and configuration of electric field. The 'classical aging effects', well known since the advent of wire chambers, lead to the formation of deposits, conductive or insulating, on the electrode surfaces and manifest themselves as a decrease of the gas gain due to the modification of electric field, excessive currents, self-sustained discharges, or sparking. Traditionally, the aging rate has been parameterized as a normalized gas gain loss: $R=-\frac{1}{G} \frac{d G}{d Q}(\% \operatorname{per} C / \mathrm{cm})$, where $G$ is the initial gas gain, $d G$ is the loss of gas gain after collected charge $d Q$ per unit length [3]. However, the assumption that the aging rate is only a function of the total accumulated charge has not been confirmed for gaseous detectors operated in high-rate environments. In reality, the rate of polymer formation depends upon many microscopic variables such as cross-sections of electron and photon processes and their energy distributions in gas avalanches, molecular dissociation energies, as well as densities of electrons, ions and free radicals. Consequently, one may expect that the aging rate could also be affected by macroscopic parameters, such as gas gain, ionization density and radiation intensity, which are directly related to the basic microscopic variables. Several results presented at the 2001 workshop clearly indicate that such dependencies do exist.

Some of the conclusions from the 1986 workshop are still valid in 2001. However, the dramatic

increase in charge (up to $1.0 \frac{C}{c m \text { wire }}$ per year), which is expected to be accumulated on sensing electrodes in the new high rate experiments, poses much more stringent constraints on the radiation hardness of materials and gas mixtures, assembly procedures, and basic rules for construction and operation of gaseous detectors, than previously encountered. Only a limited choice of gases have been demonstrated to tolerate such doses. Moreover, recent experience with straws and honeycomb drift tubes revealed that chemical etching processes leading to a dramatic damage of gold-plating on wires could occur in non-polymerizing $C F_{4}$ mixtures at exceedingly high current densities. These new developments since the 1986 workshop raise a question about the adequacy of using $C F_{4}$-based 


\section{ICFA Instrumentation Bulletin}

mixtures for long-term, high-rate applications.

The scientific program of the 2001 workshop addressed specific questions which, as reported by many authors, are of a primary interest: classical aging effects, models and insights from plasma chemistry, materials for detectors and gas systems, lessons learned from detector operation at high radiation intensities, new aging effects, experiences with large systems and recommendations for future detectors. About 100 detector experts attended the 4 day workshop, and 10 invited talks, 31 contributed talks and 9 posters were presented in 7 sessions [5].

\section{General Characteristics of Aging Processes}

\subsection{Classical Aging Effects}

The 'classical' aging effects are the result of chemical reactions occurring in avalanche plasmas near anodes in wire chambers leading to formation of deposits on electrode surfaces. During gas avalanches many molecules break up in collisions with electrons, de-excitation of atoms, and UVphoton absorption processes. Whereas most ionization processes require electron energies greater than $10 \mathrm{eV}$, the breaking of covalent molecular bonds and formation of free radicals requires only 3-4 eV, and can lead to a higher concentration of free radicals than that of ions in the gaseous discharges. Consequently, free-radical polymerization is regarded as the dominating mechanism of wire chamber aging. Since free radicals are chemically very active they will either recombine to form the original molecules or other volatile species, or may start to form new cross-linked molecular structures of increasing molecular weight. When the polymerized chain becomes large enough for condensation to occur, it will diffuse to an electrode surface.

It is worthwhile to mention that one has to distinguish between formation of polymers in the gas avalanche near the anode wire and their deposition on electrode (anode or cathode) surfaces. The polymer deposition mechanism can be viewed as a phenomenon that occurs whenever the gaseous species fails to bounce back after a collision with an electrode surface, including a surface layer of molecules previously formed in the gas discharges. Initially, the polymer could be attached to the surface very weakly, unless some additional chemical reactions take place between the polymer atoms and atoms of the wire material. Moreover, many free radicals are expected to have permanent or induced dipole moments so that electrostatic attraction to a wire can also play a significant role in the polymer deposition process. For the inert gold-plated anode wires the probability for polymers to stick to the surface is rather small until the creation of the first monolayer of deposits, which may significantly increase further deposition. The influence of surface wire quality on anode aging and a model of polymer film growth is proposed in [6]. The importance of reactions between the electrode 


\section{ICFA Instrumentation Bulletin}

material and polymers produced in avalanches for the deposition mechanism can be illustrated by the following examples:

- Non-gold anode wires react with fluorine radicals produced in an avalanche to form resistive metal fluorides. Many studies have demonstrated excellent aging properties, up to $10 \frac{C}{\mathrm{~cm} \cdot \text { wire }}$, of $C_{4} / i C_{4} H_{10}$ (80:20) gas avalanches [7]-[9], which also has the ability to etch silicon-based and hydrocarbon deposits on previously aged gold-plated wires $[10,11]$. However, extensive deposition was observed on unplated wires irradiated in $C F_{4} / i C_{4} H_{10}(80: 20)[11,12]$.

- Exceedingly large aging rates were observed in pure $C F_{4}$ and in $A r / C F_{4} / O_{2}$ (50:40:10) [11][13], which are typical etching gases and reluctant to polymerize. This effect was related to the chemical processes at the cathode, where trace fluorocarbon deposits were found resulting in a loss of gas gain and not in a self-sustained Malter discharge.

The self-sustained discharge (Malter effect) [14], which is due to a thin insulating layer deposited on a conducting cathode by a polymerization mechanism, is one of the most devastating phenomena of all aging effects. The resistivity of the insulating layer defines the maximum rate capability of the detector before the onset of field-emission of electrons from the cathode, which starts if the rate of ionic charge neutralization across the dielectric film is smaller than the rate of ion charge buildup $[15,16]$. There exists evidence that certain metal oxide coatings on the cathode and/or simply the cathode material itself (eg. carbon-loaded polycarbonate foil) may not be conducting enough and could cause Malter-like breakdowns in the presence of large localized ionization densities $[3,16,17]$. Several other factors may facilitate its ignition, such as highly ionizing particles, sparks, sharp points on electrodes causing corona discharges, or thin anode wires [15]. It is easy to ignite Malter currents in a detector operating with hydrocarbon gases at elevated high voltages [18] or forcing chambers to breakdown [19]-[21], and in a detector, which has been previously exposed to TMAE gas [22]. The CRID RICH detector [23] with an excellent 3-dimensional single electron reconstruction capability allowed the first imaging of the onset of the Malter effect, which starts from sporadic bursts of single electrons from a localized cathode spot [22]. Such a positive feedback between electron emission at the cathode and anode amplification will lead to high ionization densities at distinct chamber locations. This, in turn, can initiate the production of new reactive species at much larger rates, thus promoting more deposits to form at the same cathode spot to an extent sufficient to establish a classical self-sustained Malter discharge. The most dangerous consequence of this phenomena is that the Malter effect could easily spread over a large area, if it goes undetected for a long period of operation, thus causing irreparable damages to the chamber.

Many experiments have demonstrated that the addition of $\mathrm{H}_{2} \mathrm{O}$ or alcohols - after the insulating

layer at the cathode is already formed - tends to stabilize the detector operation, but not to cure the Malter effect $[2,3]$. When these additives are removed, usually the chamber suffers from Malter 
effect again. Recently, it was discovered that the addition of oxygen $(0.02-0.05 \%)$ or $\mathrm{CO}_{2}(5 \%)$ to the damaged chamber, which showed a self-sustained dark current with $\mathrm{He} / i \mathrm{C}_{4} \mathrm{H}_{10}$ (80:20), could revert or cure a Malter breakdown in the presence of high current density [18]. When the oxygen is removed, the chamber can still operate at a high ionization level (although it will start to age again without additive). It is also worthwhile to mention that the possibility of reanimation of anode wires aged in hydrocarbon gases by means of sputtering was demonstrated in $\mathrm{Ar} / \mathrm{O}_{2}$ (99:1) and $\mathrm{Ar} / \mathrm{CO}_{2}(93: 7)[24,25]$. These effects support results from plasma chemistry, where it is known that oxygen reacts with hydrocarbon molecules and the end products are volatile $\mathrm{CO}, \mathrm{CO}_{2}, \mathrm{H}_{2} \mathrm{O}$ and $\mathrm{H}_{2}$.

\subsection{Wire Chambers vs Plasma Chemistry}

While the specific reactions responsible for wire chamber aging are extremely complex, some qualitative approach to the aging phenomena in different gases could be obtained from similarities between chemical processes in plasmas of gas avalanches $[2,3,11,12]$ and those that occur in the better-understood low-pressure ( $<1$ Torr) rf $(13.6 \mathrm{MHz})$ plasmas [26,27]. Although many parameters (electric field, gas pressure, electron density, power density) are vastly different between the two regimes, the electron energies are not so different. Also, in both cases the free radicals are most likely the active species involved in polymer formation.

In plasma polymerization, the overall mechanism of 'Competitive Ablation and Polymerization' proves to be a basic principle that describes reactions occurring in a plasma polymerization system. Considerable fragmentation of the gas molecules or rearrangement of atoms occurs in the plasma and the extent of the process and the dominating mechanism vary with the types of gases and the discharge conditions. The most important concept here is that both polymer-forming species and species that cause ablation (physical or chemical etching) of materials are created in the plasma of the original gas. The significance of this concept is fully established in perfluorocarbon plasmas, which represent the most extreme case of ablation competing with polymer formation. Actually, $C F_{4}$-based gases are used for both etching and deposition processes, the distinction being made by the gas and its concentration with which $C F_{4}$ is mixed. In general, the addition of oxygenated species shifts the chemistry of $C F_{4}$ plasmas towards etching, while the addition of hydrogenated species shifts the chemistry towards polymerization $[26,28]$. In the former case, the dissociative products of $\mathrm{CF}_{4}$ and $\mathrm{O}_{2}$ are the most desirable active species for the etching processes in plasmas [29]-[32]. For the latter case, in the absence of hydrogen, products of the $C F_{4}$ discharge could act as an effective etching gas especially for $S i$-based deposits, which react with fluorine to form volatile $\mathrm{SiF}_{4}$. The addition of hydrogen atoms or molecules to $C F_{4}$ scavenge $F$ atoms by the formation of more stable $H F$ and produces a mixture with carbon-enriched $\left(C F_{3}, C F_{2}, C F\right)$ 


\section{ICFA Instrumentation Bulletin}

residues. As the ratio of $F / C$ decreases, perfluorocarbons polymerizes readily, i.e. the balance shifts from ablation to polymerization [28,33]-[35]. For instance, very fast polymer formation was observed in $\mathrm{C}_{6} F_{6}$ and $\mathrm{C}_{2} \mathrm{H}_{2} F_{4}$ plasmas [28]. On the other hand, hydrofluoric acid can chemically attack $H F$-soluble materials existing in the system. Under certain conditions, Si-etching can be accompanied by the polymerization of the etching gas $C F_{4}$ on the $S i$-substrate $[29,36,37]$.

Correspondingly, recent results from wire chamber operation also show that both polymerization and etching phenomena can occur in $C F_{4}$-based gases (see section $\mathrm{V}$ ). Particularly, using the same experimental setup, a lack of apparant aging have been observed in $C F_{4} / i C_{4} H_{10}$ (80:20) and $C F_{4} / i C_{4} H_{10}$ (50:50) mixtures, whereas heavy carbonaceous deposits were observed on the gold-plated wires in $\mathrm{CF}_{4} / i C_{4} H_{10}$ (95:5), $\mathrm{CF}_{4} / i C_{4} H_{10}$ (20:80) and $\mathrm{CF}_{4} / \mathrm{C}_{2} \mathrm{H}_{4}$ (95:5) gases [11].

Two other examples, where conclusions from plasma chemistry are qualitatively applicable to wire chambers are:

- In plasma chemistry, most organic compounds with oxygen-containing groups are generally reluctant to form polymers. For example, water in plasmas could act as an efficient modifier of the polymer chain-growth mechanism by reacting with polymer precursors and forming volatile species (up to $50 \% \mathrm{H}_{2} \mathrm{O}$ was added to the plasma feed gas) [26]. In wire chambers, the addition of water (a few hundred to a few thousands ppm of $\mathrm{H}_{2} \mathrm{O}$ ) has been found to effectively suppress polymerization effects [38,39], to prevent Malter breakdown [18,40,41], or even to restore the original operation in aged counters $[42,43]$. There is more than one mechanism by which $\mathrm{H}_{2} \mathrm{O}$ /alcohols can help in wire chambers [15,44]. Because of the large dipole moment, these molecules will tend to concentrate near the electrode surfaces, where polymerization takes place. Water has an additional advantage in wire chambers since it increases the conductivity of the partially damaged electrodes - a property that can have adverse effects in a MSGC [4].

- In plasmas, the characteristic polymerization rate of $S i$ is higher than for $C$ [28]. From the viewpoint of wire aging, even minor traces of $S i$-pollutants in the gas have a much higher tendency to create deposits, than similar amounts of hydrocarbon molecules.

It has to be stated, though, that the absence of corresponding systematic studies in plasma chemistry with parameters similar to wire chambers (atmospheric pressure, power densities, gas mixtures) does not allow any quantitative comparisons between the plasma chemistry and wire chamber processes. 


\section{ICFA Instrumentation Bulletin}

\section{Experience from laboratory $\mathbf{R} \& \mathbf{D}$ experiments.}

Over the last few decades an impressive variety of experimental data has been accumulated from laboratory tests and detectors installed at high energy physics facilities. However, there are many contradictory experiences obtained in seemingly identical conditions, which means that we do not always control all parameters that influence aging effects. It is now well established that - even if a low aging rate can be obtained in the laboratory with very pure gas and otherwise clean conditions - large-area detectors using the same mixture can fail due to severe aging after a relatively small beam exposure. However, experience from the laboratory, where operating conditions are much better controlled, can be used to understand some general principles and might help to implement these results successfully in large chambers.

There are a lot of experiments that clearly indicate premature aging in $\mathrm{Ar} / \mathrm{CH}_{4}$ mixtures exposed to intense radiation [38,45]-[49]. Moreover, the aging rate in $\mathrm{Ar} / \mathrm{CH}_{4}$ (90:10) was found to be mainly a function of current density, i.e. the product of irradiation rate and gas gain, independently from electrode material and purity of methane [50,51]. This observation indicates that $\mathrm{CH}_{4}$ itself polymerizes in the avalanche plasma due to the hydrogen deficiency of radicals and their ability to make bonds with hydrocarbon molecules [3,26], and similarly for all hydrocarbon gases. Under certain conditions the aging rate in $\mathrm{Ar} / \mathrm{C}_{2} \mathrm{H}_{6}$ with alcohol can be strongly reduced [52,53]. However, noble gas/hydrocarbon mixtures are not trustworthy for long-term, high-rate experiments. In order to suppress polymerization of hydrocarbons, oxygen-containing molecules can be added to the mixture. For $\mathrm{Ar} / \mathrm{CH}_{4} / \mathrm{CO}_{2}$, measurements have shown that sensitivity to aging decreases with decreasing $\mathrm{CH}_{4}$ and increasing $\mathrm{CO}_{2}$ content [24].

Dimethylether (DME) appeared in the 1986 workshop as a good quencher and a reasonably good radiation-hard gas for wire chambers operated at high intensities. The aging rate in DME tends to be lower than the polymerization rate of ordinary hydrocarbons [2] and several groups reported the absence of aging effects in wire chambers up to large values of accumulated charge $[6,50,51,54]$. However, the aging effects in DME appear to be highly sensitive to traces of pollutants at the ppb level, which are difficult to keep under control in large detectors. There is also evidence of high chemical reactivity of DME, which requires a careful material selection for detector assembly and gas system components [55].

Attempts were made to replace organic quenchers with aging resistant ones, like $\mathrm{CO}_{2}$. The $\mathrm{Ar}(\mathrm{Xe}) / \mathrm{CO}_{2}$ gases could be in principle absolutely radiation resistant under clean conditions; up to now, there is no well-established mechanism, which could lead to the formation of anode deposits in these mixtures. Stable operation up to $\sim 1 \frac{\mathrm{C}}{\text { cm wire }}$ was reported for $\mathrm{Ar} / \mathrm{CO}_{2}[2,3,56,57]$ and up to $\sim 5 \frac{\mathrm{C}}{\mathrm{cm} \text { wire }}$ for $\mathrm{Xe} / \mathrm{CO}_{2}$ mixtures [58]. However, a gradual decomposition of $\mathrm{CO}_{2}$ can also occur and the resulting pure carbon can be deposited specifically at the cathode [24,59]. Sometimes this 


\section{ICFA Instrumentation Bulletin}

carbon layer does not affect the performance of drift tubes [24]. Recent systematical aging tests were performed for the ATLAS muon aluminum drift tubes. In order to guarantee reproducibility of the results and to study aging behavior under different operating conditions, 26 tubes with $\mathrm{Ar} / \mathrm{CO}_{2}$ (93:7)+600 ppm $\mathrm{H}_{2} \mathrm{O}$ mixture have been irradiated with an $\mathrm{Am}^{241}$ source up to an accumulated charge of $\sim 1.3 \frac{\mathrm{C}}{\mathrm{cm} \text { wire }}$ and 47 tubes with $\mathrm{Ar} / \mathrm{CO}_{2}$ (90:10) gas were exposed to a $\mathrm{Cs}^{137}$ source up to $\sim 0.6 \frac{C}{c m \text { wire }}[25,60,61]$. All tubes were $100 \%$ efficient at the end of these aging runs, however, these measurements represented an average performance of the wire over a length of $3 \mathrm{~m}$ and were not sensitive to local inefficiencies. It should be mentioned, that the aging performance of $\mathrm{Ar} / \mathrm{CO}_{2}$ is sensitive to traces of impurities. Si-based pollutants are one of the sources of aging in $\mathrm{Ar} / \mathrm{CO}_{2}$, probably due to the production of non-volatile $\mathrm{SiO}_{2}[57,60,62,63]$. Several other experiments also observed aging effects in $\mathrm{Ar} / \mathrm{CO}_{2}$, however, the reasons of the gain reduction were not identified $[6,25,64,65]$.

The identification of radicals and fragments formed in the electron avalanches is a means to understand and eventually overcome the problems related to the aging of gaseous detectors [3,6668]. A recent investigation of avalanche products has shown that seventeen new compounds were identified in the effluent gas stream from an irradiated proportional counter with $\mathrm{Ar} / \mathrm{C}_{2} \mathrm{H}_{4}$ (50:50) mixture [69]. Some of the observed species (aliphatic hydrocarbons) contained double or triple bonds which, similar to plasma polymerization, can be easily 'opened' in the discharges and polymerize very aggressively. The systematic analysis of light emission spectra in proportional counters may also provide useful information about basic physics processes in electron avalanches [70,71].

\section{Experience with 'standard radiation level' detectors.}

\subsection{Classical Wire Chambers.}

For a long time, classical wire chambers of various designs with many thousands of wires have been used for large-area tracking detectors in the 'standard radiation levels' experiments, i.e. with total collected charges $<50 \mathrm{mC} / \mathrm{cm}$ for the whole period or running. Basic rules for the construction and operation of these detectors are known. Moreover, many of the large wire chambers were built and demonstrated to work $[2,3,72]$. Nevertheless, recent experience with large systems still shows the appearance of aging effects associated mainly with hydrocarbon polymerization and with presence of pollutants in the gas system.

A time expansion chamber filled with $\mathrm{CO}_{2} / \mathrm{iC}_{4} \mathrm{H}_{10}$ (80:20) mixture successfully operated as a vertex detector of the L3 experiment at LEP. After an accumulated charge of $10^{-4} \frac{C}{c m}$ wire collected during 11 years of running, there was no sign of aging [73]. Classical aging effects (Malter effect or/and sense wire deposits) have been observed in the H1 Central Jet Chamber operated 


\section{iCFA Instrumentation Bulletin}

with $\mathrm{Ar} / \mathrm{C}_{2} \mathrm{H}_{6}(50: 50)+0.1 \% \mathrm{H}_{2} \mathrm{O}$ and in the ZEUS Central Tracking Detector filled with $\mathrm{Ar} / \mathrm{CO}_{2} / \mathrm{C}_{2} \mathrm{H}_{6}(83: 12: 5)+0.5 \% \mathrm{C}_{2} \mathrm{H}_{5} \mathrm{OH}$ at the HERA ep-collider. In the former case, the replacement of $0.1 \% \mathrm{H}_{2} \mathrm{O}$ with $0.8 \%$ of ethanol cured the Malter effect and stabilized the detector operation [74], while for the latter case the aging problem was alleviated by the addition of $\mathrm{H}_{2} \mathrm{O}$ [75]. In both systems there was no clear indication that polymerization is 'fed' by the presence of impurities in the gas system, indicating that hydrocarbons are the likely source of chamber aging.

An abundance of literature exists describing the dramatic effect of certain gaseous constituents, which may be either due to the contaminants initially present in the gas system or that result from outgassing of construction materials upon the aging rate of wire chambers. Several examples of large systems, where the presence of pollutants increased the aging rate many times, have been reported in $[76,77]$. While laboratory tests with prototype chamber indicated negligible aging rates $(R<10 \% / C / \mathrm{cm})$, a much larger aging rate $(1000 \% / C / \mathrm{cm})$ was observed in the large Central Tracking Chamber of the CDF experiment operated with $\mathrm{Ar} / \mathrm{C}_{2} H_{6}(50: 50)+0.1 \%$ alcohol. The analysis of the aged sense wires showed the presence of $\mathrm{C}, \mathrm{O}$ and $\mathrm{Si}$ elements. After cleaning the gas system components and making changes to reduce aerosols emanating from an alcohol bubbler, the aging rate was greatly reduced allowing the detector to operate without dramatic loss in performance. The presence of $S i$ is to be pointed out here since silicon has been systematically detected in analysis of many wire deposits, although in many cases the source of Si-pollutant has not been clearly identified. The Si-compounds are found in many lubricants, adhesives and rubber, encapsulation compounds, silicon-based grease, various oils, G-10, RTV, O-rings, fine dust, gas impurities, polluted gas cylinders, diffusion pumps, standard flow regulators, molecular sieves, and their presence may not necessarily be noted in the manufacturer's documentation [2,3,51]. Because of a high specific polymerization rate $S i$ molecules should be avoided in the detector system at all cost. Consequently, if there is a question whether or not some device may incorporate silicon compounds it should be subjected to additional aging test. Another example is the D0 WAMUS muon drift chambers, which suffered fast anode aging when operated with an $\mathrm{Ar} / \mathrm{CF}_{4} / \mathrm{CO}_{2}$ (90:6:4) mixture. Here, the source of the contaminant was outgassing of glass-steel polyester epoxy resin used in the construction. A cold-trap added to the gas recirculating system reduced the aging rate by a factor of ten, while the extreme method of quickly heating the wires just below their melting point ('zapping') succeeded in blowing hardened sheaths of outgassing products off of the wires; thus completely cleaning the aged gold wires 'in-situ' [77].

The chosen examples underline the importance of having control over all detector parameters, but often it is quite difficult to draw final conclusions since nominally identical detectors connected to the same gas circuit may perform very differently [74,77]. In some cases it might be possible to eliminate harmful impurities by installing appropriate filters or cold traps in the gas system [76,77]. Many helpful guidelines for construction and operation of classical wire chambers at 'low' rates, 


\section{ICFA Instrumentation Bulletin}

which have been compiled over the past 40 years, are summarized in [78] as follows:

- Create a moderately clean environment during detector construction and clean the gas system components prior to start of operation;

- Avoid the presence of 'bad' molecules in contact with active gas ( $S i$, halogens, sulphur, plasticizers, outgassing) [2,3];

- A huge variety of gases can be successfully used (noble gases, hydrocarbons, freons, $\mathrm{CO}_{2}$, $D M E, H_{2} O$, alcohols, 'magic gas', ...);

- Hydrocarbons are the most likely source of aging (effect is more pronounced in presence of contamination or under discharges, sparks, Malter effect). Even with addition of water/alcohol the improvements are still limited and it is problematic to consider them for high rate applications;

- If aging effects are observed despite taking all of the above precautions, add suitable additives and/or identify the source of pollution and clean the gas system.

\subsection{Resistive Plate Chambers.}

In the 1990's, Resistive Plate Chambers (RPC) were proposed as an economical and proven technology ideally suited for large-area detection systems. For example, both the Belle and BaBar experiments have instrumented their flux returns with RPC's operated in streamer mode. However, high chamber currents started to show up in Belle's RPC's almost immediately upon installation. This problem was related to the presence of high levels of water $(\sim 2000 \mathrm{ppm})$ in the gas, which permeated through the walls of the polyethylene tubing. Operating the RPC's with $\mathrm{Ar} / \mathrm{C}_{4} \mathrm{H}_{10} / \mathrm{C}_{2} \mathrm{H}_{2} \mathrm{~F}_{4}$ (30:8:62) plus water in streamer mode led to the formation of hydrofluoric acid that etched the electrodes made from ordinary glass. This resulted in the creation of emission points triggering chamber currents. The problem was finally solved by replacing the plastic tubes with copper ones $\left(\mathrm{H}_{2} \mathrm{O}<10 \mathrm{ppm}\right)[79]$.

In the BaBar RPC's the electrodes are made of Bakelite coated with linseed oil. After an initial period of successful operation with an $\mathrm{Ar} / \mathrm{C}_{4} \mathrm{H}_{10} / \mathrm{C}_{2} \mathrm{H}_{2} \mathrm{~F}_{4}$ mixture, the RPC's started to show a permanent reduction in efficiency and an increase in dark current. The main conclusion of the subsequent extensive $R \& D$ studies related the BaBar RPC problem to the lack of polymerization of the linseed oil and formation of oil droplets under the influence of high temperature and high currents [80]. Further efficiency deterioration mechanisms, that may play an important role in BaBar's RPC's have been proposed by J. Va'vra [15]. He suggested that this problem could be due to an electrochemical change of resistivity of fresh linseed oil, modulated by the presence of water in the RPC's. A positive example is the L3 RPC at LEP, which operated at a very low 


\section{ICFA Instrumentation Bulletin}

particle flux over 8 years without significant loss of efficiency [81]. In contrary to RPC's used in streamer mode at the Belle, BaBar and L3 experiments, the future LHC experiments will operate RPC's in proportional mode, which is desirable in terms of total accumulated charge per particle. However, much higher particle fluxes at the LHC require more systematic $R \& D$ studies of the RPC technology, since many processes could degrade their performance under high-rate conditions. Recent results of aging tests for ATLAS, CMS and LHC-B RPC's indicate that under the right circumstances RPC can withstand large integrated doses [82]-[84].

It should be stressed though that the problems with Belle and BaBar RPC's are not 'classical aging effects', but rather unpredictable surface effects, related to the specific choices of materials and operating conditions.

\subsection{Gaseous Photodetectors.}

Gaseous photon detectors used in high energy physics experiments must ensure an efficient way of converting $U V$ photons to electrons with a subsequent detection of single photoelectrons $[85,86]$. For gaseous converters, systematical aging studies have been carried out with TMAE and TEA vapors [87]-[89], which are added to the carrier gas to provide photoionization capability [90]. TMAE is the best material in terms of quantum efficiency, however, the main obstacle of using TMAE at high rates is an exceedingly rapid gas gain loss due to deposits on the anode wires [86,91][94]. Several studies have indicated that anode wire deposits can be removed by heating the wires with elevated currents [89,95]; unfortunately this recovery is followed by a quick drop in gain [96,97]. In addition, all photosensitive materials, and, most probably, their various aging products are good insulators and may excite self-sustained currents when deposited on the cathode [86]. Systematic studies with TMAE and TEA also allowed to establish basic dependencies between the aging behavior and dissociation energy or wire diameter [91]:

- aging rate for $T M A E$ is larger than for $T E A$ (TMAE molecule is more fragile than $T E A$ );

- aging rate for $T M A E$ and $T E A$ is inversely proportional to the anode wire diameter;

At low rates, the possibility to use gaseous photon detectors on a very large scale at long-term with hydrocarbon/TMAE gases has been demonstrated for large $4 \pi$ devices (e.g. SLD CRID and DELPHI RICH) [86]. It is worthwhile to mention, that the high reactivity of TMAE with oxygen and other substances necessitates a very high degree of cleanliness and leak-tightness for gas systems in these detectors.

In recent years, there has been considerable work in the field of photon imaging detectors by combining solid photocathodes $(C s I)$ and wire chambers or gaseous electron multipliers. However, several aging tests also revealed degradation of CsI photocathode quantum efficiency for very high rate environments; a collection of existing aging data for these can be found in [86,91,92,98]-[101]. 
iCFA Instrumentation Bulletin

\section{Aging Experience with High-Rate detectors of the LHC era.}

The most recent developments in high-energy physics require a dramatic increase of the radiation intensity encountered by gaseous detectors: from $\mathrm{mC} / \mathrm{cm} /$ wire for the 'standard radiation level' detectors up to $C / \mathrm{cm} /$ wire for the new high-rate experiments of the LHC era (HERA-B, LHC). Among the most critical items that affect the lifetime of gaseous detectors (apart from the gas mixtures) are the materials in contact with gas, assembly procedures, gas mixing and distribution systems, and tubing. In section III.A we discuss the outgassing properties of several materials and general rules for assembly of high-rate gaseous detectors and gas systems, while sections III.B-D contain the summary of the recent experience with aging problems in gaseous detectors operated at extremely large particle fluxes.

\subsection{Choice of Materials for Detectors and Gas Systems}

The increasingly challenging requirements for building and testing the next generation of large-area gaseous detectors has demanded a concerted effort towards finding adequate materials for detectors and associated gas systems. Many non-metallic 'good materials' successfully used in the 'standard radiation level' detectors might nevertheless outgas at a small level, thus causing fast aging under high rate conditions. The lifetime studies of Microstrip Gas Chambers (MSGC) in high intensity environments, which also had the greatest impact on the understanding of aging phenomena in all types of gaseous detectors, demonstrate that the amount of pollutants in the gas system play a major role in determining the aging properties of the detector [4]. Consequently, the outgassing from materials, epoxies, joints, tubing, etc. has to be carefully controlled. For obvious reasons, the use of glues, plastics and many organic materials is unavoidable in particle detectors. It is therefore very important to choose materials, which are suitable for the practical mechanical and electrical assembly of a gaseous detectors, in terms of their possible outgassing effects and radiation robustness.

It is suggested to start by searching for low-outgassing materials in a NASA database, which was originally developed for selecting spacecraft materials [51,102]. It contains more than 1600 entries for adhesives, 500 entries for rubbers and elastomers, 800 entries for potting materials. This list can help to pre-select assembly materials before doing tests matching the specific requirements of each detector.

A large amount of outgassing data for epoxy compounds, adhesive tapes, leak sealers, rigid materials, O-rings, and plastic pipes have been accumulated in the framework of the RD-10 project at CERN, which afterwards was merged with the more specific research on MSGC within the 


\section{iCFA Instrumentation Bulletin}

RD-28 project. In the RD-10 tests, an outgassing box, placed upstream of the strongly irradiated wire counter, was used to introduce samples of materials into the gas stream, thus allowing a systematic study of outgassing effects on the chamber lifetime. Furthermore, the gas flowing from the chamber was analyzed with a Gas Chromatograph (GC) and Mass Spectrometer (MS) or Electron Capture Device (ECD). While for some materials only outgassing properties were verified and materials releasing detectable pollutants were rejected, for other 'radiation hard materials' full evidence of suitability was obtained in long-term aging tests of a validated clean detector. The long list of candidates recommended to be used in the construction of gaseous detectors can be found in [2]-[4],[103]-[106] and was summarized at the 2001 workshop [51]. The effect of outgassing from materials on the lifetime of gaseous detector can be illustrated by several examples: Araldit AW103 epoxy mixed with HY991 hardener did not induce any detectable gas pollutants in the GC/MS and was also validated by irradiating the wire chamber. It is presently used as a glue for the construction of ATLAS straws [106] and triple-GEM stations of the COMPASS experiment [107]. The GC/MS analysis of another popular epoxy, Araldit106 and HV953 U, extensively used in the assembly of older MWPC's, revealed traces of heavy hydrocarbon molecules in the effluent gas stream, which could be partially responsible for the observation of aging reported in $[17,60]$. In fact, this glue has shown the largest outgassing rate among all tested glues in [106]. Interestingly, outgassing can also be due to an incorrect ratio of hardener to resin or even insufficient curing time; both factors may largely increase the gas contamination $[51,108]$.

Gas tubes used for the supply of the active gas have always been the object of primary attention when analyzing aging effects in wire chambers. Electro-polished stainless steel and hydrogen-fired copper gas pipes are the best choices for gaseous detectors operated in high-rate environments, since they are free of outgassing and ensure zero gas permeability. However, due to their high price and concerns for the material budget in the active area of the detector, many experiments often use cheaper plastic tubes, although these are susceptible to outgassing, have high gas permeability and can consequently cause severe aging. Particularly, PVC, Teflon and neoprene rubber tubes contain halogen atoms in molecular chains, which are known to increase drastically the aging rate [2,3]. Polyethylene tube outgasses water, large alcanes and substituted aromatics [67]. One of the classical examples, cited by many authors, shows that the introduction of PVC pipe can initiate a gain reduction, which continues with the same rate even after the PVC tube is replaced with the original stainless steel tubes [20]. This indicates a potentially very serious problem: one can cause permanent damage to a detector by a wrong choice of material even for a limited period of time. Therefore, one should use as much steel as possible for gas supply lines, especially in parts exposed to high radiation doses.

Up to now, there is no strong objection to the use of nylon (polyamide, RILSAN) tubes if they are not too long. However, plastic pipes usually introduce water to the gas due to the 


\section{ICFA Instrumentation Bulletin}

natural outgassing and/or due to the diffusion of air humidity through the walls: as much as $\sim 1700 \mathrm{ppm}$ of water can be added to the gas by placing $20 \mathrm{~m}$ of nylon pipe at the chamber inlet [51]. Particularly, nylon tubes were used to introduce water indirectly to the chamber for curing Malter breakdown [40]. However, the presence of water can cause 'bad' surface chemistry as described above and is therefore extremely dangerous for certain RPC and MSGC detectors.

The general recommendations concerning the choice of assembly materials and rules for the mechanical construction of high-rate detectors, which includes adequate assembly procedures, personnel training, quality checks, final testing as part of fighting against the aging, have been reported at the workshop in $[51,78]$. There are clearly many 'bad' and a lot of usable materials. However, a specific material is either adequate or not for a particular detector type and operating conditions one has to do tests matching the specific requirements of the experiment. Finally, no spontaneouslychosen materials should be installed in the detector or gas system in the last minute, before the start of real operation.

\subsection{Micro-pattern Gas Detectors.}

Future high-luminosity experiments have prompted a series of inventions of new high-rate gaseous detectors: MSGC, MICROMEGAS, Gas Electron Multipliers (GEM) and many others $[109,110]$. The systematic research of the physical parameters used to manufacture and operate MSGC's, such as substrate and assembly materials, metal for the strips, and type and purity of the gas mixtures play a dominant role in determining their long-term stability $[4,111,112]$. Despite the promising performance of MSGC's (high-rate capability, good space accuracy, and excellent multi-track resolution), there are several major processes, particularly at high rates, leading to operating instabilities: charge-up of substrates, destructive microdischarges, and surface deposition of polymers [4].

The influence of glass conductivity has been verified for MSGC's: the use of borosilicate glass as a substrate results in rate-dependent modification of gain due to the radiation induced variation of surface resistivity. Use of electron-conducting or diamond-coated glass solves the problems of short and long-term instabilities for detectors made on insulating support [4]. The problem of microdischarges, induced by heavily ionizing particles and destroying the electrode structure, turned out to be a major limitation to all single-stage micro-pattern detectors in hadronic beams $[113,114]$. The nature and resistivity of the strip material affects the developments of sparks [115]. Aluminum electrodes are more robust against gas discharges than gold. However, the use of $A l$ electrodes led to the appearance of 'bubbles' or 'craters' on the cathode strips even at modest collected charges, while no aging effects were observed with strips made from gold [116].

Microstrip chambers have been operated with a large variety of gases; to prevent fast aging at high rates, convincing evidence suggests again to avoid hydrocarbons in the gas $[117,118]$. Under 


\section{ICFA Instrumentation Bulletin}

optimal laboratory conditions, absence of any degradation of MSGC performance with $\operatorname{Ar} / D M E$ has been demonstrated by many groups up to large accumulated charges [4,112,118]-[121]. However, MSGC-GEM detectors operated with $A r / D M E$ shows fast aging under $X$-rays, if the size of the irradiated area is large enough, while identical chambers with $\mathrm{Ar} / \mathrm{CO}_{2}$ showed no aging $[116,122]$. Long-term survival without degradation has been also observed for triple-GEM and MICROMEGAS-GEM detectors, operated with $\mathrm{Ar} / \mathrm{CO}_{2}$ [123,124]. Unfortunately, $\mathrm{Ar} / \mathrm{CO}_{2}$ mixtures have worse quenching properties and are more prone to discharges than $A r / D M E$. Protection against sparking can be significantly improved by adding a small amount $(\sim 0.3 \%)$ of $\mathrm{H}_{2} \mathrm{O}$ to the MSGC. However, in contrary to wire chambers, such an addition of water led in one case to massive coating on the anode strips both in $\mathrm{Ar} / \mathrm{DME}$ and $\mathrm{Ar} / \mathrm{CO}_{2}$ mixtures [4,116]. These observations underline the importance of careful selection of materials and gas mixtures for highrate applications and of treating micro-pattern detectors as delicate devices during production and running phases.

\subsection{Choice of Gas Mixtures.}

Future high energy and luminosity experiments pose a new challenge for gas mixtures, raising the requirement for their radiation hardness up to $\sim 1 \frac{C}{c m \text { wire }}$ per year. Under these constraints only a limited choice of gases is available, and from the 'conventional mixtures' only $\mathrm{Ar}(\mathrm{Xe}) / \mathrm{CO}_{2}$ is demonstrated to tolerate such doses. Unfortunately, these mixtures are quite transparent for photons and have a low electron drift velocity, which limits their possible application for high-rate detectors and large drift distances.

About twenty years ago, $C F_{4}$ was proposed as the most attractive candidate for high-rate environments [125]-[127]. This is primarily due to the high-drift velocity, high primary ionization, low electron diffusion and resistance to aging $[13,128,129]$. Within the broad spectrum of gas mixtures, there is no gas mixture without $C F_{4}$ that is able to tolerate doses $\sim 10 \frac{C}{c m \text { wire }}$. It is believed that, when $C F_{4}$ dissociates in the gaseous discharges into highly reactive $C F_{x}$ and $F$ radicals, the atomic fluorine is very effective in suppressing polymerization effects. However, $\operatorname{Ar}(\mathrm{Xe}) / \mathrm{CF}_{4}$ mixtures have rather poor energy and spatial resolution due to the dissociative electron attachment processes in $C F_{4}$ [130]- [132]. Moreover, the $C F_{4}$ molecule has a small quenching cross-sections of metastable $A r$-states [133] and excited $C F_{4}$ molecules emit photons from the far UV to the visible [134]. This results in an intolerable level of afterpulsing in $A r / C F_{4}$ gases even at moderate gas gains. The advantage of the enhanced drift velocity of $C F_{4}$ for high-rate applications have been realized by the addition of one of the common quenchers (e.g. $\mathrm{CO}_{2}, \mathrm{CH}_{4}$ ) to $\mathrm{CF}_{4}$ or to $\mathrm{Ar} / \mathrm{CF}_{4}$. This can also 'cool' electrons to the extent that attachment does not occur. 


\section{ICFA Instrumentation Bulletin}

\subsection{Lessons learned from detector operation at high ionization densi- ties.}

Accelerated aging tests, often carried out with radioactive sources or $X$-rays to emulate the longterm lifetime properties of the detector, can be extrapolated to the real experimental conditions directly only if the aging rate depends on the total collected charge and not upon the current density, particle rate, or gas gain at which the dose was accumulated. In reality, many laboratory studies have demonstrated severe gain losses at lower charge accumulation rates, other conditions being held constant $[4,7,20,42,48,50]$. The lower polymerization rate for higher current densities is attributed to the onset of space charge effects, which reduce the electron energies in the avalanche, thus decreasing the density of ions and radicals in the avalanche plasma.

The new generation of high-rate detectors of the LHC era has not only to cope with high dose rates, but also has to survive in a hostile presence of heavily ionizing particles with an average energy deposition 10-1000 times larger than for MIP's. An exposure of 'large-scale' gaseous detectors over the full area in such a harsh radiation environment at high ionization densities $(>100 \mathrm{nA} / \mathrm{cm})$ can result in greatly enhanced polymer formation: an abundance of aggresive radicals will diffuse for rather long times ( $\sim$ hours) within the irradiated chamber and will react with other avalancheproduced polymer fragments. According to this naive picture, this mechanism could significantly accelerate polymerization in large systems, whereas in small-scale laboratory tests the aging rate typically decreases with increasing gas flow $[3,7,8,25]$. Furthermore, polymer deposition in wire chambers likely starts from localized spots and then can spread over the entire irradiated region. Since in large, mass-produced systems an extremely high quality for electrode surfaces and cleanliness of the gas systems are hard to reach, any imperfections - in the presence of high currents could easily trigger sparks, discharges or Malter currents, which will in turn dramatically increase the polymerization rate.

Recent systematic research clearly demonstrates that the initial stage of radiation tests usually performed in the laboratory may not offer the full information needed to estimate the lifetime of the real detector. Strong dependence of aging performance upon size of the irradiated area, current density, high voltage (gas gain), irradiation rate, particle type and energy have been observed in high-rate environments.

Severe anode and cathode aging effects were found in prototype honeycomb drift tubes operated with $\mathrm{CF}_{4} / \mathrm{CH}_{4}$ (80:20) and $\mathrm{Ar} / \mathrm{CF}_{4} / \mathrm{CH}_{4}$ (74:20:6) mixtures in the high-rate HERA-B environment (secondaries from interactions of $920 \mathrm{GeV}$ proton with target nucleus) after a few $\mathrm{mC} / \mathrm{cm}$ of accumulated charge [16,17,135-137]. This effect was surprising since chambers had previously been proven to be immune to very large $X$-ray doses up to $5 \mathrm{C} / \mathrm{cm}$. An extensive $R \& D$ program, carried out at 10 different radiation facilities to resemble HERA-B conditions, revealed that $X$-rays 


\section{iCFA Instrumentation Bulletin}

or electrons were not able to trigger Malter currents, while in the large-area modules, irradiated with hadrons above a certain energy, Malter effect appeared very rapidly. The aging effects in these honeycomb drift tubes were traced to a combination of several problems; a solution which uses gold-coated cathode foils and a $\mathrm{Ar} / \mathrm{CF}_{4} / \mathrm{CO}_{2}$ (65:30:5) mixture was found to survive in a high-rate hadronic environment up to $\sim 1 \frac{C}{c m \text { wire }}[17]$.

The aging performance of the HERA-B muon proportional chambers has been studied with $\mathrm{Ar} / \mathrm{CF}_{4} / \mathrm{CH}_{4}, \mathrm{CF}_{4} / \mathrm{CH}_{4}$, and $\mathrm{Ar} / \mathrm{CF}_{4} / \mathrm{CO}_{2}$ mixtures in a variety of conditions [138]-[141]. The aging rate in $\mathrm{Ar} / \mathrm{CF}_{4} / \mathrm{CH}_{4}$ was found to be more than two orders of magnitude higher in hadronic beams than in the laboratory studies with radioactive sources. In addition, strong dependences of the aging properties on high voltage and progressive deterioration of the gas gain in the direction of the serial gas flow have been observed for large-scale prototypes irradiated with $\mathrm{Ar} / \mathrm{CF}_{4} / \mathrm{CH}_{4}$ (67:30:3) in the harsh HERA-B environment. Aging effects increasing in the direction of the serial gas flow (even outside the irradiation zone) have been also reported for a $\mathrm{Ar} / \mathrm{CO}_{2} / \mathrm{C}_{2} \mathrm{H}_{2} \mathrm{~F}_{4}$ mixture [142]. A strong dependence of the aging properties on high voltage, irradiation rate, length of irradiation and gas flow rate has been also observed in the ATLAS muon drift tubes operated with $\mathrm{Ar} / \mathrm{CH}_{4} / \mathrm{N}_{2} / \mathrm{CO}_{2}$ (94:3:2:1) [24,25,60,61]. Here, the systematic $R \& D$ studies have shown a nearly exponential dependence of chamber lifetime on high voltage and on the counting rate within the experimentally investigated parameter range. Unfortunately, the high voltage is not the physical quantity directly responsible for aging in wire chambers, therefore, these aging effects could be classified as depending on the gas gain and/or current density, which are related to the density of ions and radicals in the avalanche plasma.

Dependence of polymer formation on the energy input is well established in plasma polymerization. Nearly all organic compounds regardless of their chemical nature can be polymerized under certain conditions. The structure of plasma polymers formed from the same monomer is highly dependent on the actual conditions of polymerization: the energy input level, the size (cross-sectional area) of a tube and even on the position within the reactor [26]. The experimental dependence of chamber lifetime on ionization density, gas gain and irradiation rate, which are also related to the total dissipated energy in the detector from ionizing particles, indicates that the aging behavior can not be solely explained on the basis of the molecule ratios in the mixture (gas composition), without taking into account the actual operating conditions. These results illustrate the need for studying the aging performance of a detector under conditions as close as possible to the real environment.

The dependence of the detector lifetime on the size of the irradiated area, in particular, and the increase of the aging rate in the direction of the serial gas flow means that aging should be viewed as a non-local and intensity-dependent phenomenon. These observations seem to be the most critical when trying to extrapolate the aging behavior from laboratory tests to large-scale detectors. Some of the long-lived aggressive radicals may diffuse in the direction of the gas flow and react with other 


\section{iCFA Instrumentation Bulletin}

avalanche generated contaminants, thus enhancing aging effects with increasing usage of the gas. Here it is important to note that due to the increased aging in the direction of the gas flow it is worthwhile to avoid gas distribution systems that supply many chambers by a serial flow.
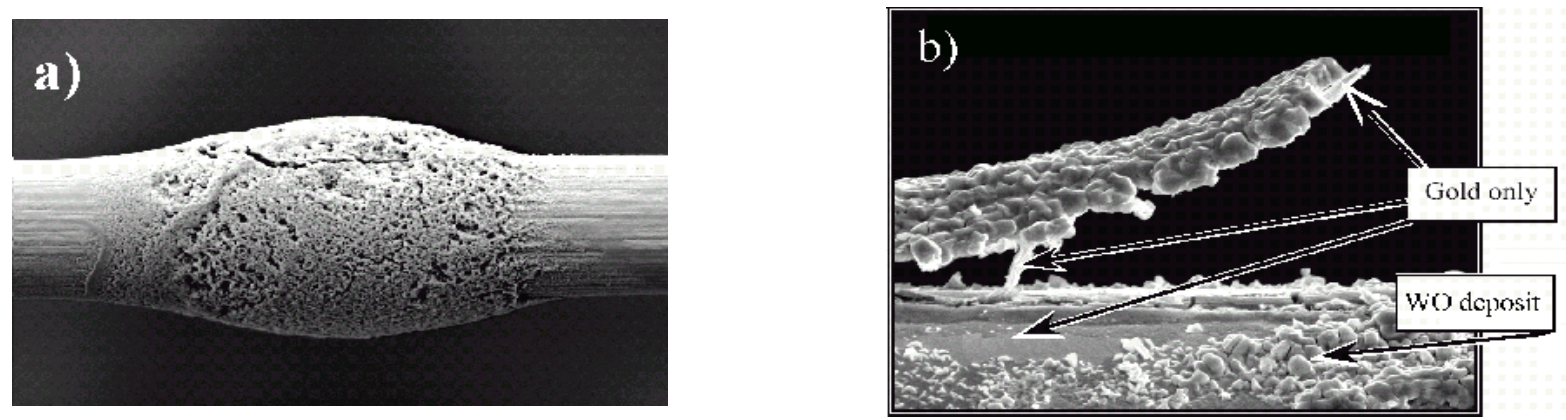

Figure 1: Various manifestations of damage to the gold-plating of wires: a) amorphous $W O$ deposits [147]; b) long piece of gold peeled away from the wire surface [148].

As reported above, polymer deposition can occur in $\mathrm{CF}_{4} / \mathrm{CH}_{4}$ mixtures, as suggested by plasma chemistry. Similarly, the aging properties of $\mathrm{Ar}(\mathrm{Xe}) / \mathrm{CF}_{4} / \mathrm{CO}_{2}$ gases, which by analogy with plasma experiments should have excellent etching properties, have been widely investigated over the last years. Using $\mathrm{Ar} / \mathrm{CF}_{4} / \mathrm{CO}_{2}$ mixtures under optimal operating conditions, no observable drop in gain due to polymerization has been found for the HERA-B honeycomb drift tubes up to $1.5 \mathrm{C} / \mathrm{cm}$ [17], HERA-B aluminum proportional chambers up to $0.7 \mathrm{C} / \mathrm{cm}$ [138], CMS cathode strip chambers up to $0.4 \mathrm{C} / \mathrm{cm}$ [143], $13 \mathrm{C} / \mathrm{cm}$ [63], LHCb multi-wire proportional chambers up to $0.25 \mathrm{C} / \mathrm{cm}$ [144], COMPASS straw tubes up to $1.1 \mathrm{C} / \mathrm{cm}$ [145] and HERMES drift tubes up to $9 \mathrm{C} / \mathrm{cm}$ [146]. Moreover, honeycomb drift tubes which were initially aged with $\mathrm{Ar} / \mathrm{CF}_{4} / \mathrm{CH}_{4}$ were afterwards successfully recovered in $\mathrm{Ar} / \mathrm{CF}_{4} / \mathrm{CO}_{2}$ [17]. However, an analysis of the cathode surfaces at the end of operation with $\mathrm{Ar} / \mathrm{CF}_{4} / \mathrm{CO}_{2}$ revealed in some cases the presence of fluorine-based deposits on the cathodes, which fortunately did not result in self-sustained currents $[63,138,143]$. Since dissociative products of $C F_{4}$ react violently with many materials and the resultant polymer films at cathodes could provoke aging effects one should seriously consider using materials in high-rate detectors which are very robust to $C F_{4}$; gold-plated electrodes [17] or straw cathode materials [147] fulfill this requirement.

A further advantage of $C F_{4}$-based mixtures is additional resistance against $S i$-polymerization. This suggestion is based on experiences from plasma polymerization, where discharges of $\mathrm{CF}_{4} / \mathrm{H}_{2}$ are successfully used for $\mathrm{SiO}_{2}$ etching, while $\mathrm{CF}_{4} / \mathrm{O}_{2}$ plasmas selectively etches $\mathrm{Si}$ [29]. Extensive studies performed for the ATLAS straws partially confirm this hypothesis: $S i$-deposits have not been observed in irradiation area for large current densities $(>1 \mu \mathrm{A} / \mathrm{cm})$. On the other hand, $\mathrm{SiO} / \mathrm{SiO}_{2}$-deposits were found at the edges and even outside of the irradiated area. The resulting balance between $S i$ polymerization and $C F_{4}$ etching processes was found to be very sensitive to 


\section{ICFA Instrumentation Bulletin}

the $S i$-source intensity and ionization density [147].

In the most recent investigations at extremely high current densities $(\sim 1-5 \mu \mathrm{A} / \mathrm{cm})$ in $\mathrm{Ar}(\mathrm{Xe}) / \mathrm{CF}_{4} / \mathrm{CO}_{2}$ mixtures a new 'aging phenomenon' has appeared - the damage of the goldplating of wires in straws and honeycomb drift tubes. Several years of intensive research at CERN with straw tubes under different conditions and for different types of wires indicate that the main components responsible for gold wire damage in $\mathrm{Xe} / \mathrm{CF}_{4} / \mathrm{CO}_{2}$ (70:20:10) are harmful radicals, products of $\mathrm{CF}_{4}$ disintegration, in connection with $\mathrm{H}_{2} \mathrm{O}$. (It was suggested that $\mathrm{HF}$ acid could be responsible for destruction of gold-plating and formation of $W O$ deposits). No wire damage effects have been observed for water concentrations below $0.1 \%$ up to $20 \mathrm{C} / \mathrm{cm}$ ! [147]. Dedicated studies with straw tubes performed at PNPI with $\mathrm{Xe} / \mathrm{CF}_{4} / \mathrm{CO}_{2}$ (70:20:10) and $\mathrm{Ar} / \mathrm{CF}_{4} / \mathrm{CO}_{2}$ (60:30:10) mixtures demonstrated that under high dose rates the gold-plating of the wire was cracked, the wire diameter increased and a large amount of oxygen was observed on the tungsten in gold cracks [148][150]. Similar effects have been observed for wires irradiated in a $\mathrm{Ar} / \mathrm{CO}_{2} / \mathrm{C}_{2} \mathrm{H}_{2} \mathrm{~F}_{4}$ mixture [142]. The authors propose a model to explain the results - an anode wire 'swelling' mechanism, where the forces causing the damage to the wire surface develop under the gold layer of the wire [149]. Fig. 1 shows examples of a variety of wires with damaged gold-plating from $[147,148]$. In contrary to the experience with straws, in one test with honeycomb drift tubes irradiated with $\mathrm{Ar} / \mathrm{CF}_{4} / \mathrm{CO}_{2}$ (65:30:5) the destruction of gold coating and even rupture of anode wires have been observed only for water concentration below $50 \mathrm{ppm}$, while for $\mathrm{H}_{2} \mathrm{O}>400 \mathrm{ppm}$, gold wire damage effects were avoided [151,152]. Further studies still remain to be done to fully understand the exact mechanism of gold wire damage during operation at high ionization densities. Although no $F$-based deposits were observed on the anode wires in any of the tests, the chemically reactive dissociative products of $\mathrm{CF}_{4}$ most probably initiate the destruction processes of gold-plating.

Studies of straw proportional tubes with $\mathrm{Xe} / \mathrm{CF}_{4} / \mathrm{CO}_{2}$ mixture revealed another phenomenon, which might degrade detector performance in high rate experiments. The gas composition was found to be modified in the avalanche plasma of a strongly irradiated straw, presumably due to the production of some long-lived and highly electro-negative species [153]. These electro-negative radicals could be also responsible for the so-called 'transient aging effect' observed at high-rates in $\mathrm{Xe} / \mathrm{CF}_{4} / \mathrm{CO}_{2}$ [154], $\mathrm{Ar} / \mathrm{CF}_{4} / \mathrm{CO}_{2}$ [151] and $\mathrm{Ar} / \mathrm{CF}_{4} / \mathrm{CH}_{4}$ [138]. A 'transient aging effect' is a temporary gain reduction, which can be restored by an appropriate increase of the gas flow. The very high aggressiveness of dissociative products of $C F_{4}$ and the dynamic modification of the gas composition requires more detailed studies to evaluate the possible consequence of these effects on the long-term performance and stability of large-area gaseous detectors. In view of the aging results described here, one can see that the presence of large amounts of $C F_{4}$ in the mixture does not necessarily ensure good aging properties automatically.

The challenge to avoid aging in the new generation of high-rate experiments requires not only 


\section{ICFA Instrumentation Bulletin}

a very careful choice of all detector materials, but also forces the gas systems to be of previously unnecessary quality and cleanliness. However, a real system will always contain some degree of imperfection and pollution - despite all precautions. It has to be stressed here that in closed-loop recirculation systems, which are required for detectors operated with expensive gases $\left(\mathrm{Xe}, \mathrm{CF}_{4}\right)$ all impurities and reactive radical fragments will remain in the gas until they are removed by a purification system or deposited elsewhere. Therefore, for the construction of large-area gaseous detectors the maximal cleanliness for all processes and quality checks for all system parts are of primary importance. Examples of 'clean' gas systems currently used for high-rate detectors are presented in $[155,156]$. Certainly, the definition of the word 'clean' has changed considerably since the 1986 workshop.

\section{Summary}

Aging phenomena obviously constitute one of the most complex and serious problems which could limit the use of gaseous detectors in unprecedently severe radiation environments. The operation in high-intensity experiments of the LHC era demand not only an extraordinary radiation hardness of construction materials and gas mixtures, but also appropriate assembly procedures and quality checks during detector construction and testing. Since the 1986 workshop, considerable progress has been made on the understanding of general principles which might help to prevent or at least to suppress the aging rate to an acceptable level. However, a quantitative description of the aging processes, which would require a detailed knowledge of the reaction cross sections of all chemical species in the avalanche plasma, is still not available.

After the many years of intensive research and development of radiation-hard gaseous detectors, an impressive variety of experimental data has been accumulated. The radiation hardness and outgassing properties of the various materials used for the construction of detectors and gas systems are among the most crucial items affecting the lifetime of gaseous detector. However, the observed dependences of aging performance on the nature and purity of the gas mixture, different additives and trace contaminants, construction materials, gas flow, size of the irradiated area, irradiation intensity, ionization density, high voltage, particle type and energy, make quantitative comparisons of aging properties under very different conditions very difficult. Consequently, this data can serve only as a guideline before the start of long-term studies under conditions as close as possible to the real environment of the experiment. Such radiation tests should include an extended study of 'large-scale' final prototype chambers, exposed over the full area to a realistic radiation profile (particle type and energy, ionization density, irradiation rate). It is of primary importance to vary the operating parameters systematically in order to investigate their possible influence on the aging performance. In order to exclude statistical fluctuations of unknown nature and to provide 


\section{ICFA Instrumentation Bulletin}

a reliable estimate for the detector lifetime, the radiation tests should be carried out with several detectors irradiated under identical conditions.

This paper is based on the results reported at the DESY workshop, and also briefly discusses other experience with gaseous detectors relevant to the present aging problems. Transparencies and videos of presentations from the 'International Workshop on Aging Phenomena in Gaseous Detectors' (DESY, Hamburg) are available at the workshop's web-page (http://www.desy.de/agingworkshop). The proceedings of the workshop will be published in a special volume of Nuclear Instruments and Methods: Section A.

\section{Acknowledgment}

We are especially grateful to Andreas Schwarz (DESY), Jaroslav Va'vra (SLAC) and Mar Capeans (CERN) for many helpful discussions and careful reading of this manuscript.

\section{References}

[1] G. Charpak, H. Fisher, C. Gruhn, A. Minten, F. Sauli, G. Plch, G. Flugge, "Time degeneracy of multiwire proportional chambers," Nucl. Instr. Meth., vol. A99, pp. 279-284, 1972.

[2] J. Va'vra, "Review of wire chamber aging," Nucl. Instr. Meth., vol. A252, pp. 547-563, 1986.

[3] J. A. Kadyk, "Wire chamber aging," Nucl. Instr. Meth., vol. A300, pp. 436-479, 1991.

[4] R. Bouclier, M. Capeans, C. Garabatos, G. Manzin, G. Million, L. Ropelewski, et al., "Aging of microstrip gas chambers: problems and solutions," Nucl. Instr. Meth., vol. A381, pp. 289-319, 1996.

[5] Proceedings of the International Workshop on Aging Phenomena in Gaseous Detectors, to be published in Nucl. Instr. Meth. A

[6] V. Blinov, "Influence of materials and sense wire surface quality on aging with DME and other gases" presented at the International Workshop on Aging Phenomena, DESY, Hamburg, Oct. 2-5, 2001.

[7] R. Henderson, R. Openshaw, W. Faszer, M. Salomon, G. Sheffer, "Wire chamber ageing with $\mathrm{CF}_{4}$ /isobutane and argon/ethane mixtures," IEEE Trans. Nucl. Sci., vol. 35(1), pp. 477-482, 1988.

[8] R. Openshaw, R. Henderson, W. Faszer, D. Murphy, M. Salomon, G.Sheffer, "Tests of wire chamber ageing with $C F_{4}$ /isobutane (80:20), $A r / C_{2} H_{6}$ (50:50) and $A r / C_{2} H_{6} / C F_{4}$ (48:48:4)" IEEE Trans. Nucl. Sci., vol. 36(1), pp. 567-571, 1989. 


\section{ICFA Instrumentation Bulletin}

[9] J. Kadyk, J. Wise, D. Hess, M. Williams, "Anode wire aging tests with selected gases" IEEE Trans. Nucl. Sci., vol. 37(2), pp. 478-486, 1990.

[10] R. Openshaw, R. Henderson, W. Faszer, M. Salomon "Etching of anode wire deposits with $C F_{4}$ /isobutane (80:20) avalanches," Nucl. Instr. Meth., vol. A419, pp. 351-356, 1991.

[11] J. Wise, J. Kadyk, D. Hess, "Chemical model for wire chamber aging in $C F_{4} / i C_{4} H_{10}$," $J$. Appl. Phys., vol. 74(9), pp.5327-5340, 1993.

[12] J. Wise, "Chemistry of radiation damage to wire chambers," Dissertation, University of California, Berkeley, LBL-32500, 1992.

[13] J. Va'vra, P. Coyle, J. Kadyk, J. Wise, "Measurements of electron drift parameters for helium and $C_{4}$-bases gases," Nucl. Instr. Meth., vol. A324, pp. 113-126, 1993.

[14] L. Malter, "Thin film field emission," Phys. Rev., vol. 50, pp. 48-58, 1936.

[15] J. Va'vra, "Physics and Chemistry of Aging - Early developments" presented at the International Workshop on Aging Phenomena, DESY, Hamburg, Oct. 2-5, 2001.

[16] G. Bohm, "Observations on cathode aging in honeycomb drift tubes," presented at the International Workshop on Aging Phenomena, DESY, Hamburg, Oct. 2-5, 2001.

[17] C. Padilla, "Aging studies for the large honeycomb drift tube system of the outer tracker of HERA-B," presented at the International Workshop on Aging Phenomena, DESY, Hamburg, Oct. 2-5, 2001.

[18] A. Boyarski, "Laboratory aging tests for the BABAR drift chamber," presented at the International Workshop on Aging Phenomena, DESY, Hamburg, Oct. 2-5, 2001.

[19] B. Foster, "Whisker growth in test cells," Proceedings of Workshop on Radiation Damage to Wire Chambers, LBL-21170, pp. 227-229, 1986.

[20] R. Kotthaus, "A laboratory study of radiation damage to drift chambers," Proceedings of Workshop on Radiation Damage to Wire Chambers, LBL-21170, pp. 161-193, 1986.

[21] H. Sadrozinski, "Investigation of breakdown conditions of drift chambers," Proceedings of Workshop on Radiation Damage to Wire Chambers, LBL-21170, pp. 121-129, 1986.

[22] J. Va'vra, "Can TMAE photocathode be used for high rate applications?" Nucl. Instr. Meth., vol. A367, pp. 353-357, 1995.

[23] K. Abe, P. Antilogus, D. Aston, K. Baird, A. Bean, R. Ben-David, et al., "Performance of the CRID at SLD," Nucl. Instr. Meth., vol. A343, pp. 74-86, 1994.

[24] V. Pashhoff, "Studies on ageing and reanimation of drift tubes for the ATLAS muon spectrometer," Dissertation, University Freiburg, 1999. 


\section{ICFA Instrumentation Bulletin}

[25] S. Kircher, M.Kollefrath, G. Herten, W. Mohr, "Parameters of MDT ageing and reanimation," ATLAS note, ATLAS-MUON-012 (2001).

[26] H. Yasuda, "Plasma Polymerization," Academic Press, 1985.

[27] H. V. Boenig, "Plasma Science and Technology," Cornell University Press, 1982.

[28] H. Yasuda "New insights into aging phenomena from plasma chemistry," presented at the International Workshop on Aging Phenomena, DESY, Hamburg, Oct. 2-5, 2001.

[29] M.J. Kushner, "A kinetic study of the plasma-etching processes. A model for the etching of Si and $\mathrm{SiO}_{2}$ in $\mathrm{C}_{n} F_{m} / \mathrm{H}_{2}$ and $\mathrm{C}_{n} F_{m} / \mathrm{O}_{2}$ plasmas," J. Appl. Phys., vol. 53(4), pp. 2923-2938, 1982.

[30] C.J. Mogab, A.C. Adams, D.L. Flamm, "Plasma etching of $\mathrm{Si}$ and $\mathrm{SiO}_{2}$ - the effect of oxygen additions to $C F_{4}$ plasmas," J. Appl. Phys., vol. 49(7), pp. 3796-3803, 1978.

[31] H.F. Winters, J.W. Coburn, E. Kay, "Plasma etching - A 'pseudo-black-box' approach," J. Appl. Phys., vol. 48(12), pp. 4973-4983, 1977.

[32] J.C. Martz, D.W. Hess, W.E. Anderson, "Tantalum etching in fluorocarbon/oxygen rf glow discharges," J. Appl. Phys., vol. 67(8), pp. 3609-3617, 1990.

[33] E.A. Truesdale, G. Smolinsky, T.M. Mayer, "The effect of added acetylene on the rf discharge chemistry of $C_{2} F_{6}$. A mechanistic model for fluorocarbon plasmas," J. Appl. Phys., vol. 51(5), pp. 2909-2913, 1980.

[34] E.A. Truesdale, G. Smolinsky, "The effect of added hydrogen on the rf discharge chemistry of $C F_{4}, C F_{3} H$, and $C_{2} F_{6}, " J . A p p l$. Phys., vol. 50(11), pp. 6594-6599, 1979.

[35] S. Motlagh, J.H. Moore "Cross sections for radicals from electron impact on methane and fluoroalkanes," J. Chem. Phys., vol. 109(2), pp. 432-438, 1998.

[36] T. Arikado, Y. Horiike, "Si and $\mathrm{SiO}_{2}$ etching under low self-bias voltage," Jap. J. Appl. Phys., vol. 22(5), pp. 799-802, 1983.

[37] H.F. Winters, "The role of chemisorption in plasma etching," J. Appl. Phys., vol. 49(10), pp. $5165-5170,1978$.

[38] M. Kollefrath, V. Paschhoff, M. Spegel, U. Topp, C. Fabjan, G. Herten, et al., "Aging studies for the ATLAS-monitored drift tubes," Nucl. Instr. Meth., vol. A419, pp. 351-356, 1998.

[39] J.P. DeWulf, D. Geiregat, P. Vilain, F. Bergsma, C. Busi, A. Capone, et al., "Test results of the streamer-tube system of the charm II neutrino detector," Nucl. Instr. Meth., vol. A252, pp. 443-449, 1986.

[40] J. Kadyk, J. Va'vra, J. Wise, "Use of straw tubes in high-radiation environments," Nucl. Instr. Meth., vol. A300, pp. 511-517, 1998. 


\section{ICFA Instrumentation Bulletin}

[41] J.P. Venuti, G.B. Chadwick, "Radiation aging studies of $\mathrm{CO}_{2} /$ hydrocarbon mixtures for the SLD drift chamber," IEEE Trans. Nucl. Sci., vol. 36(1), pp. 595-599, 1989.

[42] A. Algeri, H.G. Fischer, S.O. Holmgren, M. Szeptycka "Anode wire ageing in proportional counters: the problem of analog response," Nucl. Instr. Meth., vol. A338, pp. 348-367, 1994.

[43] M. Danilov, N. Nagovitsin, V. Shibaev, I. Tichomirov, E. Michel, W. Schmidt-Parzefall, "Study of drift chamber aging with propane," DESY note-88-090 (1988).

[44] J. Va'vra, "Aging of gaseous detectors," SLC-PUB-5207, 1990.

[45] A. Smith, M.J.L. Turner, "Lifetime of proportional counters filled with xenon/methane and argon/methane," Nucl. Instr. Meth., vol. A192, pp. 475-481, 1982.

[46] H. Sipila, M.L. Jarvinen, "Extended lifetime of a wire chamber," Nucl. Instr. Meth., vol. A217, pp. 298-300, 1983.

[47] K. Kwong, J.C. Layter, C.S. Lindsey, S.O. Melnikoff, B.C. Shen, G.J. Vandalen, M.C. Williams, "Hydrocarbon polymerization on drift chamber wires," Nucl. Instr. Meth., vol. A238, pp. 265-272, 1985.

[48] I. Juricic, J. Kadyk, "Anode wire aging measurements and a search for remedies," IEEE Trans. Nucl. Sci., vol. 34(1), pp.481-485, $198 \%$.

[49] K. Silander, E.P. de Lima, M.M. Fraga, R.F. Marcus, F. Fraga, M. Salete Leite, A.J.P.L. Policarpo, "Aging studies with argon/methane based gas mixtures," Nucl. Instr. Meth., vol. A367, pp. 298-301, 1995.

[50] R. Bouclier, M.Capeans, C. Garabatos, R.D. Heuer, M. Jeanrenaud, T.C. Meyer, F. Sauli, K. Silander, "Results of wire chamber aging tests with $\mathrm{CH}_{4^{-}}$and DME-based gas mixtures," Nucl. Instr. Meth., vol. A346, pp. 114-119, 1994.

[51] M. Capeans, "Aging and Materials: Lessons for Detectors and Gas Systems" presented at the International Workshop on Aging Phenomena, DESY, Hamburg, Oct. 2-5, 2001.

[52] M. Atac, "Wire chamber aging," Proceedings of Workshop on Radiation Damage to Wire Chambers, LBL-21170, pp. 55-66, 1986.

[53] S. Beingessner, T. Meyer, V. Vuillemin, M. Yvert, "The UA1 central detector at present and future luminosity," Nucl. Instr. Meth., vol. A257, pp. 552-555, $198 \%$.

[54] R. Henderson, "Effects of materials on aging rates in wire chambers operated with DME," presented at the International Workshop on Aging Phenomena, DESY, Hamburg, Oct. 2-5, 2001.

[55] M. Jibaly, S. Majewski, P. Chrusch, R. Wojcik, F. Sauli, J. Gaudaen, "The aging of wire chambers filled with dimethyl ether: wire and construction materials and freon impurities," Nucl. Instr. Meth., vol. A283, pp. 692-701, 1989. 


\section{ICFA Instrumentation Bulletin}

[56] A. Dwurazny, Z. Hajduk, M. Turala, "Ageing effects in gaseous detectors and search for remedies," Proceedings of Workshop on Radiation Damage to Wire Chambers, LBL-21170, pp. 113-120, 1986.

[57] E. Conti, F. Gasparini, "Test of the wire ageing induced by radiation for CMS barrel muon chambers," Nucl. Instr. Meth., vol. A465, pp. 472-481, 2001.

[58] V. Bondarenko, V. Grigoriev, S. Zverev, A. Kruglov, I. Markina, V. Peskov, et al., "Radiation hardness studies of straw proportional tubes," CERN-PPE/91-191 (1991).

[59] V. Bawdekar, "Carbon dioxide quench action in a beryllium proportional counter," IEEE Trans. Nucl. Sci., vol. 22(1), pp. 282-285, 1975.

[60] M. Kollefrath, "Aging tests for the ATLAS muon drift tubes" presented at the International Workshop on Aging Phenomena, DESY, Hamburg, Oct. 2-5, 2001.

[61] V. Paschhoff, M. Spegel, "Aging effects studies for the ATLAS MDT's using $A r / C O_{2}$ (90:10)," ATLAS note, ATLAS-MUON-019 (1999).

[62] J. Adam, C. Baird, D. Cockerill, P.K. Frandsen, H.J. Hilke, H. Hofmann, et al., "A study of aging effects in wire chambers," Nucl. Instr. Meth., vol. A217, pp. 291-297, 1983.

[63] G. Gavrilov, "Aging studies of CMS muon chambers prototypes under high doses" presented at the International Workshop on Aging Phenomena, DESY, Hamburg, Oct. 2-5, 2001; and preprint PNPI-2442 (2001).

[64] A.R. Faruqi, H. Road, "Rapid data collection systems for time resolved $X$-ray scattering experiments," IEEE Trans. Nucl. Sci., vol. 27(1), pp. 644-648, 1980.

[65] T. Kowalski, "A study of aging effect in gas monitoring proportional counters of the BAC calorimeter of the ZEUS experiment," presented at the International Workshop on Aging Phenomena, DESY, Hamburg, Oct. 2-5, 2001.

[66] D. Hess "Plasma chemistry in wire coating," Proceedings of Workshop on Radiation Damage to Wire Chambers, LBL-21170, pp. 15-23, 1986.

[67] J. Wise, J. Kadyk, D. Hess, M. Williams, "Study of plasma chemistry in wire chambers by GC/MS," IEEE Trans. Nucl. Sci., vol. 37(2), pp.470-477, 1990.

[68] J. Va'vra, "Wire chamber gases," Nucl. Instr. Meth., vol. A323, pp. 34-47, 1992.

[69] K. Kurvinen, "Analysis of organic compounds formed in electron avalanches in a proportional counter filled with ar $/ \mathrm{C}_{2} \mathrm{H}_{4}$ mixture," presented at the International Workshop on Aging Phenomena, DESY, Hamburg, Oct. 2-5, 2001.

[70] T.J. Sumner, G.K. Rochester, P.D. Smith, J.P. Cooch, R.K. Sood, "Scintillating drift chambers - the nature of the emission process in $\mathrm{Ar} / \mathrm{CH}_{4}$, IEEE Trans. Nucl. Sci., vol. 29(5), pp. 14101414, 1982. 
[71] M.M. Fraga, E.P. Lima, M.A. Alves, J. Escada, R.F. Marques, M.S. Leite, A. Policarpo, "Fragments and radicals in gaseous detectors," Nucl. Instr. Meth., vol. A323, pp. 284-288, 1992.

[72] A.V. Zarubin, "Properties of wire chamber gases," Nucl. Instr. Meth., vol. A283, pp. 409-422, 1989.

[73] B. Betev, "Experience with the L3 vertex drift chamber at LEP," presented at the International Workshop on Aging Phenomena, DESY, Hamburg, Oct. 2-5, 2001.

[74] C. Niebuhr, "Aging effects in Central Jet Chamber of H1," presented at the International Workshop on Aging Phenomena, DESY, Hamburg, Oct. 2-5, 2001.

[75] D. Bailey, "Experience with the ZEUS Central Tracking Detector," presented at the International Workshop on Aging Phenomena, DESY, Hamburg, Oct. 2-5, 2001.

[76] M. Binkley, "Experience with the Central Tracking Chambers of CDF," presented at the International Workshop on Aging Phenomena, DESY, Hamburg, Oct. 2-5, 2001.

[77] T. Marshall, "Restoring contaminated wires, removing gas contaminants and aging studies of drift tube chambers," presented at the International Workshop on Aging Phenomena, DESY, Hamburg, Oct. 2-5, 2001.

[78] B. Schmidt, "Recommendationd for building and testing the next generation of gaseous detectors," presented at the International Workshop on Aging Phenomena, DESY, Hamburg, Oct. 2-5, 2001.

[79] D. Marlow, "Recent experiences with aging in RPC's," presented at the International Workshop on Aging Phenomena, DESY, Hamburg, Oct. 2-5, 2001.

[80] D. Piccolo "Aging effects in the RPC's of the BaBar experiment," presented at the International Workshop on Aging Phenomena, DESY, Hamburg, Oct. 2-5, 2001.

[81] G. Carlino "Aging studies with L3 RPC's at LEP," presented at the International Workshop on Aging Phenomena, DESY, Hamburg, Oct. 2-5, 2001.

[82] G. Aielli "Further advances in aging studies of RPC's," presented at the International Workshop on Aging Phenomena, DESY, Hamburg, Oct. 2-5, 2001.

[83] G. Pugliese, "Aging studies for the RPC's of the CMS Muon Trigger Detector," presented at the International Workshop on Aging Phenomena, DESY, Hamburg, Oct. 2-5, 2001.

[84] G. Passaleva, "First results of an aging test of a prototype RPC for the LHCb muon system," presented at the International Workshop on Aging Phenomena, DESY, Hamburg, Oct. 2-5, 2001. 


\section{ICFA Instrumentation Bulletin}

[85] J. Seguinot, T. Ypsilantis, "A historical survey of Ring Imaging Cherenkov counters," Nucl. Instr. Meth., vol. A343, pp. 1-29, 1994.

[86] J. Va'vra, "Photon detectors," Nucl. Instr. Meth., vol. A371, pp. 33-56, 1996.

[87] C.L. Woody, "Aging effects in low pressure wire chambers containing TMAE," IEEE Trans. Nucl. Sci., vol. 35(1), pp. 493-497, 1988.

[88] J. Va'vra, "Wire aging of hydrocarbon gases with TMAE additions," IEEE Trans. Nucl. Sci., vol. $34(1), p p .486-490,198 \%$.

[89] J. Va'vra, "Wire aging with the TEA photocathode," Nucl. Instr. Meth., vol. A387, pp. 183$185,1997$.

[90] J. Va'vra, J. Kadyk, J. Wise, P. Coyle, "Study of photosensitive mixtures of TMAE and helium, hydrocarbon of $\mathrm{CF}_{4}$-based carrier gases," Nucl. Instr. Meth., vol. A370, pp. 352-366, 1996.

[91] J. Va'vra, "Photon detectors with gas amlification," Nucl. Instr. Meth., vol. A387, pp. 137-145, 1995.

[92] P. Krizan, S. Korpar, M. Staric, A. Stanovnik, M. Cindro, R. Pestotnik, et al., "Photon detectors for HERA-B RICH," Nucl. Instr. Meth., vol. A387, pp. 146-149, 1995.

[93] J. Pyrlik, M. Atiya, D. Broemmelsiek, Th. Hamacher, M. Ispiryan, S. Korpar, et al., "Aging measurements of TMAE based photon detector for the HERA-B RICH," Nucl. Instr. Meth., vol. A414, pp. 170-181, 1998.

[94] K. Lau, "Test-beam aging studies of a TMAE prototype for the HERA-B RICH," presented at the International Workshop on Aging Phenomena, DESY, Hamburg, Oct. 2-5, 2001.

[95] J. Va'vra, T. Bienz, F. Bird, M. Gaillard, G. Hallewell, Y. Kwon, et al., "Construction and initial operation of a proportional wire detector for use in a cherenkov ring imaging system," IEEE Trans. Nucl. Sci., vol. 35(1), pp. 487-492, 1988.

[96] S. Korpar, P. Krizan, A. Stanovnik, M. Staric, D. Skrk, "Aging and rejuvenation of a TMAE+methane multiwire photon detector," IEEE Trans. Nucl. Sci., vol. 46(3), pp. 31\%320, 1999 .

[97] D. Skrk, "Study of in-situ heating of aged anode wires," presented at the International Workshop on Aging Phenomena, DESY, Hamburg, Oct. 2-5, 2001.

[98] A. Breskin, "CsI photocathodes: history and mystery," Nucl. Instr. Meth., vol. A371, pp. 116-136, 1996.

[99] J. Va'vra, A. Breskin, A. Buzulutskov, R. Chechik, E. Shefer "Study of CsI photocathodes: volume resistivity and ageing," Nucl. Instr. Meth., vol. A387, pp. 154-162, 1995. 


\section{ICFA Instrumentation Bulletin}

[100] V. Peskov "Aging in gaseous photodetectors," presented at the International Workshop on Aging Phenomena, DESY, Hamburg, Oct. 2-5, 2001.

[101] A. Di Mauro, "Aging of large area CsI photocathodes for the ALICE HMPID prototypes," presented at the International Workshop on Aging Phenomena, DESY, Hamburg, Oct. 2-5, 2001.

[102] http://epims.gsfc.nasa.gov/og/index.cgi

[103] R. Bouclier, M.Capeans, C. Garabatos, F. Sauli, K. Silander, "Effects of outgassing from some materials on gas chamber aging," Nucl. Instr. Meth., vol. A350, pp. 464-469, 1994.

[104] R. Bouclier, M. Capeans, C. Garabatos, G. Manzin, G. Million, L. Ropelewski, F. Sauli, et al., "Aging studies with microstrip gas chambers," Nucl. Phys. B., vol. 44, pp. 557-566, 1995.

[105] A. Romaniouk, "Choice of materials for the construction of the TRT," ATLAS-INDET/98211 (1998).

[106] F. Guarino, S. Ilie, A. Romaniouk, S. Soutchkov, G. Tardelli "Outgassing studies of materials for the TRT construction," ATLAS-INDET/99-011 (1999).

[107] B. Ketzer, S. Bachmann, M. Deutel, J. Friedrich, S. Kappler, I. Konorov, et al., "GEM Detectors for COMPASS," IEEE Trans. Nucl. Sci., vol. 48(4), pp. 1065-1069, 2001.

[108] F. Sauli, "Review of workhop results on the fundamental understanding of aging processes," presented at the International Workshop on Aging Phenomena, DESY, Hamburg, Oct. 2-5, 2001.

[109] "Proceedings of the International Workshop on Micropattern Detectors," Orsay, France (1999).

[110] "Proceedings of the PSD99 - 5th International Conference on Position Sensitive Detectors," London, England (1999).

[111] A. Barr, S. Bachmann, B. Boimska, R. Bouclier, A. Braem, C. Camps, et al., "Construction, test and operation in a high intensity beam of a small system of micro-strip gas chambers," Nucl. Instr. Meth., vol. A403, pp. 31-56, 1998.

[112] F. Sauli, A. Sharma, "Micro-Pattern Gaseous Detectors," CERN-EP/99-69 (1999).

[113] Y. Bagaturia, O. Baruth, H. Dreis, F. Eisele,I. Gorbunov, W. Gradl, et al., "Studies of aging and HV breakdown problems during development and operation of MSGC and GEM detectors for the Inner Tracking System of HERA-B," submitted for publication to Nucl. Instr. Meth.,

[114] A. Bressan, M. Hoch, P. Pagano, L. Ropelewski, F. Sauli, S. Biagi, et al., "High rate behavior and discharge limits in micro-pattern detectors," Nucl. Instr. Meth., vol. A424, pp. 321-342, 1998. 


\section{ICFA Instrumentation Bulletin}

[115] B. Schmidt, "Microstrip gas chambers: Recent developments, radiation damage and longterm behavior," Nucl. Instr. Meth., vol. A419, pp. 230-238, 1998.

[116] M. Hildebrandt, "Aging studies for the Inner Tracker of HERA-B," presented at the International Workshop on Aging Phenomena, DESY, Hamburg, Oct. 2-5, 2001.

[117] R. Bouclier, J. Florent, J. Gaudaen, G. Million, A. Pasta, F. Sauli, et al., "High flux operation of microstrip gas chambers on glass and plastic supports," Nucl. Instr. Meth., vol. A323, pp. 240-246, 1992.

[118] L. Alunni, R. Bouclier, G. Fara, C. Garabatos, G. Manzin, G. Million, et al., "Performance of MSGC on electronically and ionically conductive substrata in various operational conditions," Nucl. Instr. Meth., vol. A348, pp. 344-350, 1994.

[119] R. Bouclier, M. Capeans, C. Garabatos, G. Manzin, G. Million, L. Ropelewski, et al., "Development of micro-strip gas chambers for high-rate operation," Nucl. Instr. Meth., vol. A367, pp. 168-172, 1995.

[120] E. Daubie, "Aging tests of MSGC detectors," presented at the International Workshop on Aging Phenomena, DESY, Hamburg, Oct. 2-5, 2001.

[121] J. Miyamoto, J. Shipsey, "An aging study of semiconductive microstrip gas chambers and a gas electron multiplier," Nucl. Instr. Meth., vol. A367, pp. 168-172, 1995.

[122] T. Hott, "Aging problems of the Inner Tracker of HERA-B: An example for new detectors and new effects," presented at the International Workshop on Aging Phenomena, DESY, Hamburg, Oct. 2-5, 2001.

[123] S. Kappler, "Aging measurements with the gas electron multiplier (GEM)," presented at the International Workshop on Aging Phenomena, DESY, Hamburg, Oct. 2-5, 2001.

[124] J. Miyamoto, "Aging Study of a MICROMEGAS with GEM preamplification," presented at the International Workshop on Aging Phenomena, DESY, Hamburg, Oct. 2-5, 2001.

[125] L. Christophorou, D. McCorkle, D. Maxey, J. Carter, "Fast gas mixtures for gas-filled particle detectors," Nucl. Instr. Meth., vol. A163, pp. 141-149, 1979.

[126] L. Christophorou, P. Datskos, J. Carter, "Gases of possible interest to SSC muon detectors," Nucl. Instr. Meth., vol. A309, pp. 160-168, 1991.

[127] L. Christophorou, J. Olthoff, M. Rao, "Electron interactions with $C F_{4}$, , J. Phys. Chem. Ref. Data, vol. 25(5), pp. 1341-1387, 1996.

[128] B. Schmidt, S. Polenz, "Electron motion in counting gases - new answers and new questions," Nucl. Instr. Meth., vol. A273, pp. 488-493, 1988. 


\section{ICFA Instrumentation Bulletin}

[129] J. Fischer, A. Hrisoho, V. Radeka, P. Rehak, "Proportional chambers for very high counting rates based on gas mixtures of $C F_{4}$ with hydrocarbons," Nucl. Instr. Meth., vol. A238, pp. 249-264, 1985.

[130] P.G. Datskos, J.G. Carter, L.G. Christophorou, "Ionization coefficients in selected gas mixtures of interest for particle detectors," J. Appl. Phys., vol. 71(1), pp. 15-21, 1978.

[131] W.S. Anderson, J.C. Armitage, E. Dunn, J.G. Heinrich, C. Lu, K.T. McDonald, et al., "Electron attachment, effective ionization coefficient, and electron drift velocity for $\mathrm{CF}_{4}$ gas mixtures," Nucl. Instr. Meth., vol. A323, pp. 273-279, 1992.

[132] S. Biagi, "Accurate three-dimensional simulation of straw chambers using slow, medium and fast gas mixtures," Nucl. Instr. Meth., vol. A310, pp. 133-136, 1991.

[133] J.E. Velazco, J.H.Kolts, D.W. Setser, "Rate constants and quenching mechanisms for the metastable states of argon, krypton and xenon," J. Chem. Phys., vol. 69(10), pp. 4357-4373, 1978 .

[134] A. Pansky, A. Breskin, A. Buzulutskov, R. Chechik, V. Elkind, J. Va'vra, "The scintillation of $C F_{4}$ and its relevance to detection science," Nucl. Instr. Meth., vol. A354, pp. 262-269, 1995.

[135] H. Kolanoski, "Investigation of aging in the HERA-B Outer Tracker Drift Tubes," Proceedings of Nuclear Science Symposium and Medical Imaging Conference, France, October 2000.

[136] M. Hohlmann, "The Outer Tracker of HERA-B," Nucl. Instr. Meth., vol. A461, pp. 21-24, 2001.

[137] C. Stegmann, "The Outer Tracker of HERA-B," Nucl. Instr. Meth., vol. A453, pp. 153-158, 2001.

[138] M. Titov, "Aging Studies for the Muon Detector of HERA-B," presented at the International Workshop on Aging Phenomena, DESY, Hamburg, Oct. 2-5, 2001.

[139] M. Danilov, L. Laptin, I. Tichomirov, M. Titov, Yu. Zaitsev, "Aging tests of the proportional wire chambers using $\mathrm{Ar} / \mathrm{CF}_{4} / \mathrm{CH}_{4}$ (74:20:6), $\mathrm{Ar} / \mathrm{CF}_{4} / \mathrm{CH}_{4}$ (67:30:3) and $\mathrm{Ar} / \mathrm{CF}_{4} / \mathrm{CO}_{2}$ (65:30:5) mixtures for the HERA-B Muon Detector," hep-ex/0107080, preprint ITEP-43-00, 2000.

[140] A. Arefiev, S. Barsuk, M. Danilov, V. Eiges, M. Titov, Yu. Zaitsev, et al., "A Gaseous Muon Detector at the HERA-B Experiment," IEEE Trans. Nucl. Sci., vol. 48(4), pp. 1059-1064, 2001.

[141] M. Danilov, Yu. Gilitsky, T. Kvaratschellia, L. Laptin, I. Tichomirov, M. Titov, Yu. Zaitsev, "Aging studies of large area proportional chambers under high rate irradiation with $C F_{4}$-based mixtures," hep-ex/0111077 and hep-ex/0111078, preprint ITEP-15-01, 2001. 


\section{ICFA Instrumentation Bulletin}

[142] G. Gavrilov, A. Krivchitch, E. Kuznetsova, V. Lebedev, L. Schipunov, E. Lobachev, "Aging properties of the straw drift-tubes operating with gas mixture containing $\mathrm{C}_{2} \mathrm{H}_{2} \mathrm{~F}_{4}$," Preprint PNPI-2407, 2001.

[143] O. Prokofiev, "Aging tests of full scale CMS muon cathode strip chambers," presented at the International Workshop on Aging Phenomena, DESY, Hamburg, Oct. 2-5, 2001.

[144] V. Souvorov, "First results of an aging test of full scale MWPC for the LHC-B muon system," presented at the International Workshop on Aging Phenomena, DESY, Hamburg, Oct. 2-5, 2001.

[145] W. Dunnweber "Irradiation response of straw drift tubes," presented at the International Workshop on Aging Phenomena, DESY, Hamburg, Oct. 2-5, 2001.

[146] J. Brack, J. Belz, S. Clark, J. Ely, B. Fox, G. Hofman, et al., "The HERMES forward tracking chambers: construction, operation and aging effects," Nucl. Instr. Meth., vol. A469, pp. 47-54, 2001.

[147] A. Romaniouk, "Aging studies for the ATLAS transition radiation tracker (TRT)," presented at the International Workshop on Aging Phenomena, DESY, Hamburg, Oct. 2-5, 2001.

[148] A. Krivchitch "Anode wire swelling - a new phenomenon for anode wire aging at high doses," presented at the International Workshop on Aging Phenomena, DESY, Hamburg, Oct. 2-5, 2001.

[149] T. Ferguson, G. Gavrilov, A. Egorov, A. Krivchitch, E. Kuznetsova, V. Lebedev, et al., "Anode wire swelling - a possible phenomenon in anode wire aging under high-accumulated doses," Preprint PNPI-2406, 2001.

[150] T. Ferguson, G. Gavrilov, A. Krivchitch, E. Kuznetsova, V. Lebedev, L. Schipunov, "The effect of oxygen on anode wire swelling under the high accumulated doses," Preprint PNPI2443, 2001.

[151] A. Schreiner, "Humidity dependence of anode corrosion in HERA-B outer tracker chambers with $\mathrm{Ar} / \mathrm{CF}_{4} / \mathrm{CO}_{2}$," presented at the International Workshop on Aging Phenomena, DESY, Hamburg, Oct. 2-5, 2001.

[152] A. Schreiner, "Aging studies of drift chambers of the HERA-B outer tracker using $\mathrm{CF}_{4}$-based gases," Dissertation, University Humboldt, 2001.

[153] M. Capeans, C. Garabatos, R. Heuer, R. Mackenzie, T. Meyer, F. Sauli, K. Silander, "Ageing properties of straw proportional tubes with $\mathrm{Xe} / \mathrm{CF}_{4} / \mathrm{CO}_{2}$ gas mixture," CERN-PPE/93-136, 1993. 


\section{ICFA Instrumentation Bulletin}

[154] V. Bondarenko, B. Dolgoshein, V. Grigoriev, A. Kruglov, I. Markina, "Studies of radiation aging of the straw proportional tubes with $\mathrm{Xe} / \mathrm{CF}_{4} / \mathrm{CO}_{2}$," Nucl. Phys. B., vol. 44, pp. 577-580, 1995.

[155] M. Hohlmann, "A clean gas system with closed loop for a large gaseous detector operating at high rates," presented at the International Workshop on Aging Phenomena, DESY, Hamburg, Oct. 2-5, 2001.

[156] H.B. Dreis, "Gas support systems for hadronic high-rate detectors," presented at the International Workshop on Aging Phenomena, DESY, Hamburg, Oct. 2-5, 2001. 


\title{
Studies of aging and HV break down problems during development and operation of MSGC and GEM detectors for the Inner Tracking System of HERA-B ?
}

\author{
Y. Bagaturia ${ }^{d}$ O. Baruth ${ }^{b, h}$ H. B Dreis ${ }^{\text {a,i }}$ F. Eisele ${ }^{a}$ \\ I. Gorbunov ${ }^{b}$ S. Gradl ${ }^{\text {a }}$ W. Gradl a S. Hausmann ${ }^{\mathrm{a}, \mathrm{j}}$ \\ M. Hildebrandt ${ }^{a, e}$ T. Hott ${ }^{a, f}$ S. Keller ${ }^{b, k}$ C. Krauss ${ }^{a}$, \\ B. Lomonosov ${ }^{d, n}$ M. Negodaev ${ }^{d, n}$ C. Richter ${ }^{a, m}$ P. Robmann ${ }^{c}$ \\ B. Schmidt ${ }^{\mathrm{a}, \mathrm{g}}$ U. Straumann ${ }^{\mathrm{a}, \mathrm{e}}$ P. Truö $\mathrm{l}^{\mathrm{c}}{ }^{\mathrm{S}}$ S. Visbeck ${ }^{\mathrm{a}, \ell}$ \\ T. Walter' C. Werner ${ }^{\mathrm{a}}$ U. Werthenbach ${ }^{\mathrm{b}} \mathrm{G}^{\mathrm{G}}$ Zech $^{\mathrm{b}}$ T. Zeuner $^{\mathrm{b}}$ \\ M. Ziegler ${ }^{a, e}$

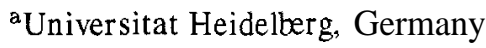
${ }^{b}$ Universitat Siegen, Germany
cUniversitat Zurich, Switzerland
dDESY, Hamburg
enow at Universitat Zurich
f now at CERN, Geneva
$\mathrm{g}_{\text {now }}$ at DESY, Hamburg
$\mathrm{h}_{\text {now }}$ at Universitat Bonn
${ }^{i}$ now at SAP, Walldorf
${ }^{j}$ now at Hewlett-Packard, Hamburg
${ }^{k}$ now at Bosch, Stuttgart
$\ell_{\text {now }}$ at Siemens, Munchen
$\mathrm{m}_{\text {now }}$ at McKinsey, Frankfurt \\ ${ }^{n}$ On leave of absence from LIP Moscow, Russia
}

\begin{abstract}
The results of ve years of development of the inner tracking system of the HERA$\mathrm{B}$ experiment and rst experience from the data taking period of the year 2000 are reported. The system contains $\mathbf{1 8 4}$ chambers, covering a sensitive area of about $(20 \times 20) \mathrm{cm}^{2}$ each. The detector is based on microstrip gas counters (MSGCs) with diamond like coated (DLC) glass wafers and gas electron multipliers (GEMs).
\end{abstract}


The main problems in the development phase were gas discharges in intense hadron beams and aging in a high radiation dose environment. The observation of gas discharges which damage the electrode structure of the MSGC led to the addition of the GEM as a rst ampli cation step. Spurious sparking at the GEM cannot be avoided completely. It does not a ect the GEM itself but can produce secondary damage of the MSGC if the electric eld between the GEM and the MSGC is above a threshold depending on operation conditions. We observed that aging does not only depend on the dose but also on the spot size of the irradiated area. Ar-DME mixtures had to be abandoned whereas a mixture of $70 \% \mathrm{Ar}$ and $30 \% \mathrm{CO}_{2}$ showed no serious aging e ects up to about $40 \mathrm{mC} / \mathrm{cm}$ deposited charge on the anodes. $\mathrm{X}$-ray measurements indicate that the DLC of the MSGC is deteriorated by the gas ampli cation process. As a consequence, long term gain variations are expected. The Inner Tracker has successfully participated in the data taking at HERA-B during summer 2000.

Key words: MSGC, MSGC-GEM, Gas aging, Discharges, HERA-B

PACS: 29.40.G, 29.40.C, 07.85.F, 81.40.C, 52.80

\section{Introduction}

The HERA-B experiment [1] was designed with the goal to measure CP violation in the B-system. It started operation at DESY, Hamburg in spring 2000. Neutral B-Mesons are produced by interactions of $920 \mathrm{GeV}$ protons on a stationary nuclear target followed by a magnetic spectrometer. Since the B cross section is very low, the experiment was planed for an interaction rate of $40 \mathrm{MHz}$ to obtain an acceptable $\mathrm{B}$ production rate. The detectors therefore were designed to withstand the corresponding high particle rates and high radiation levels.

To cope with the high particle flux which drops roughly as one over the distance from the beam axis squared, the main tracking system has been subdivided into two parts with different rate capabilities: the Inner Tracker (ITR) near the beam pipe and at larger distance the Outer Tracker (OTR) consisting of drift chambers composed of honeycomb structures.

The outer dimensions of the sensitive area of the ITR are given by the requirement that the occupancy per channel of the OTR does not exceed a critical value of $20 \%$. The high intensity area can be covered by four MSGC detectors with wafer size of $25 \times 25 \mathrm{~cm}^{2}$ which can be realized in industry. The basic geometry of a MSGC detector is shown in Figure 1. The detectors have a round recess which permits to position four MSGCs around the beam pipe. They have to sustain a particle flux of up to $2 \cdot 10^{6} / \mathrm{cm}^{2} / \mathrm{s}$ and radiation doses of up to $1 \mathrm{Mrad} /$ year. These requirements are very similar to those of the inner tracking in the ATLAS and CMS experiments for LHC and are a big challenge for the tracking

\footnotetext{
Work supported by Bundesministerium fur Bildung und Forschung, Deutschland, and the Swiss National Science Foundation.
} 


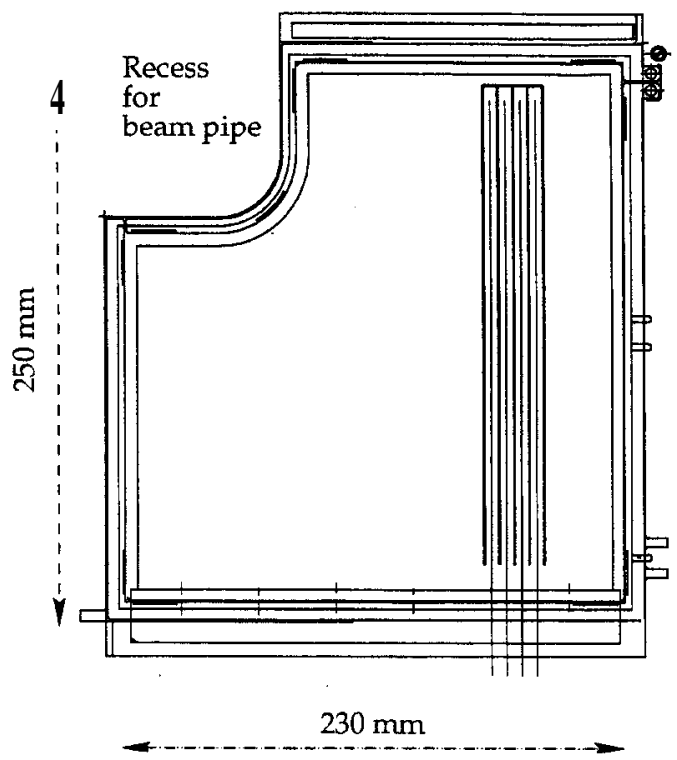

Fig. 1. Sketch of a MSGC chamber indicating size and shape of the wafer.

detector technology.

The total HERA-B ITR system requires 46 detector planes, each consisting of four chambers. Each of the 184 chambers has 752 anode strips leading to a total of about 140000 readout channels. This basic design has remained unchanged, the detector technology, however, had to be modified several times as a consequence of R\&D work and, especially, the results of beam tests.

At the time of approval of the experiment in 1995, the MSGC technology was claimed to be ready for high rate applications [2-4]. Very promising test results had been obtained and published with the claim that MSGCs on ordinary borosilicate glass wafers can be operated at high rates with good stability and homogeneous response. Moreover, aging tests with Ar-DME (argon-dimethylether) mixtures and clean gas systems were reported which were interpreted to demonstrate that these detectors can survive up to 10 years of LHC operation (equivalent to about the same time at HERA-B) without severe problems. Finally, the production of large size wafers with small error rate at rather low cost was promised.

The construction of an inner tracker for HERA-B based on the MSGC technology therefore looked rather straight forward when the experiment was approved. In the course of our development work we faced however several serious and unexpected problems:

- The production of large size MSGC wafers $25 \times 25 \mathrm{~cm}^{2}$ with acceptable quality and a small fraction of broken anodes and cathodes turned out to be difficult. After extensive R\&D at the company IMT ${ }^{1}$, series production of these wafers now has high yield with less than $5 \%$ interrupted anodes and no shorts.

- MSGCs based on bare glass (DESAG 263) wafers as proposed were unable to satisfy the

$\overline{1 \text { IMT, }}$ Greifensee, Switzerland 
requirements on gain homogeneity, rate stability and radiation tolerance. These problems were solved by the introduction of "diamond like coating" (DLC) which provides a well defined electronic surface conductivity of the substrate.

$\boldsymbol{o}$ MSGCs with DLC exhibit gas discharges between anodes and cathodes when operated in a hadron beam at the required high gas gain. Every discharge damages electrodes and in the long run this effect leads to an intolerable number of broken anodes. This problem could be solved by the introduction of an additional amplification step using the GEM technology [5].

$o$ The MSGC/GEM technology is vulnerable. We have experienced a series of problems with gas discharges at the GEM which subsequently lead to damages of the MSGC electrodes.

$\boldsymbol{o}$ Moreover, we were repeatedly faced with aging problems due to traces of impurities which are very hard to avoid reliably during production of a large series and during long term operation. Above all, the Ar-DME mixture originally foreseen as counting gas in most MSGC developments showed rapid aging in large area X-ray and test beam illumination of detectors. Finally, an $\mathrm{Ar}-\mathrm{CO}_{2}$ mixture had to be used.

In the following, major development steps and the most important results are described.

\section{The MSGC detector}

\subsection{Detector geomety}

The electrode structure chosen for the MSGC (type A) has a pitch of $300 \mathrm{~m}$, anode width of $10 \mathrm{~m}$ and an anode cathode gap of $60 \mathrm{~m}$. 48 chambers (type B) located at the downstream end of the tracking have to cover a larger sensitive area. They have a pitch of $350 \mathrm{~m}$ allowing to use the same readout configuration as for the smaller chambers. The pitch is a compromise between cost of the electronics and maximum tolerable occupancy. The relatively large anode width was chosen to minimize the number of anodes interrupted during production. The maximum length of an anode in the experiment is $\mathbf{2 3} \mathrm{cm}$. The height of the drift gap was chosen to he $3.3 \mathrm{~mm}$. This guarantees that all primary electrons have reached the anodes within $60 \mathrm{~ns}$ even for slow Ar-DME mixtures and that the anode signal occupies only one bunch crossing. Subsequent bunch crossings are $96 \mathrm{~ns}$ apart.

The high voltage is distributed via a resistor network to groups of 16 cathodes. In this way, a short between an anode and a cathode strip leads to an inefficient region of about $2 \%$ of the total chamber area.

\subsection{Wafers and coating}

Experience with first prototypes of MSGCs [6] showed that bare glass with its relatively low ionic conductivity cannot be used in a high rate and high dose environment. Local gain variations up to a factor of three were found even at low counting rates. The gas 
gain was rate dependent and the detectors did not survive an X-ray dose equivalent to about half a HERA-B year. Glass with electronic conductivity was excluded both by cost and its short radiation length. The obvious solution was to coat the surface with an electronically conducting compound. Many different coatings have been tested by various groups. Finally, DLC proved to be adequate.

DLC surfaces had been developed simultaneously by the CERN group together with the company SURMET ${ }^{2}$, and by the Siegen group together with a Fraunhofer Institut ${ }^{3}$. The two coatings are very similar in their chemical composition. They consist of mixtures of the elements $\mathrm{C}, \mathrm{Si}, \mathrm{H}, \mathrm{N}$ deposited on the glass by chemical vapor deposition (CVD) using gas mixtures of $\mathrm{CH}_{4}, \mathrm{SiH}_{4}, \mathrm{~N}_{2}$. In fact, the coating consists mainly of amorphous carbon and has very little diamond content, if any. The relative abundance of nitrogen and hydrogen and the production temperature determine the electric conductivity. Silicon improves the adhesion of the coating layer which has a typical thickness of $80 \mathrm{~nm}$. The resistivity of the coating can be increased by tempering the wafers. Tempering also increases the stability of the resistivity with time. Wafers coated by SURMET were delivered with typical resistivity of $10^{15} \Omega=\square$ whereas the resistivity of the Fraunhofer coating was originally lower by one to two orders of magnitude.

The SURMET coating had reasonable electrical properties and very good chemical stability. The mechanical quality of the large area coating was however not satisfactory most likely due to problems in handling and cleaning. For the main series production the DLC was therefore produced by the Fraunhofer Institut on AF45 glass (alkali "free", produced by DESAG ). This type of glass with little alkali content was chosen to reduce the risk of electrolytic modifications of the substrate, caused by the continuous alkali ion current over several years $[7]$. The coating has an average surface conductivity of $10^{14} \Omega=\square$ which varies over the area by about $20 \%$.

The lithographic production of the electrode pattern was realized by the company IMT on the coated wafers of $0.4 \mathrm{~mm}$ thickness and outer dimensions of about $30 \times 30 \mathrm{~cm}^{2}$.

To avoid overlapping hits in subsequent bunch crossings, we have to avoid electronic broadening of the signals propagating along the anode beyond about $50 \mathrm{~ns}$. The signal shape at the amplifier input depends on the distance of the avalanche to the amplifier end of the anode and on the product of anode-cathode resistivity and capacitance. Delta shaped voltage pulses generated at the far end of the anode produce exponentially decaying signals at the amplifier input with a time constant $=4 \mathrm{RC}={ }^{2}$, with $\mathrm{R}$ the total anode resistance and $\mathrm{C}$ the anode cathode capacitance. Thus the signal width is proportional to the strip length squared. For the given anode strip length of $23 \mathrm{~cm}$ and anode cathode capacitance of $20 \mathrm{pF}$ an anode material of resistivity ${ }^{4}$ below $250 \Omega / \mathrm{cm}$ is required. This requirement excludes nickel and chromium and points to aluminium or gold. Aluminium is preferable for lithography and has also rather good resistance to sparks (see Section 2.4). Aging tests with aluminium wafers however were discouraging (see Section 4). The aluminium option was therefore abandoned. A $500 \mathrm{~nm}$ thick gold MSGC pattern

2 SURMET Corporation, Burlington, MA, USA.

3 Fraunhofer Institut fur Schicht- und Ober achentechnik, Braunschweig, Germany.

4 The actual resistivity of lithographically produced electrodes is usually much higher than the nominal table values for bulk material. 


\begin{tabular}{|c|c|c|c|c|}
\hline material & $\begin{array}{c}\text { AF45 } \\
\text { glass substrate }\end{array}$ & $\begin{array}{c}\text { CVD } \\
\text { coating }\end{array}$ & $\begin{array}{c}\text { titanium } \\
\text { adhesion layer }\end{array}$ & $\begin{array}{c}\text { gold } \\
\text { electrode structure }\end{array}$ \\
\hline thickness & $400 \mu \mathrm{m}$ & $0.080 \mu \mathrm{m}$ & $0.050 \mu \mathrm{m}$ & $0.500 \mu \mathrm{m}$ \\
\hline
\end{tabular}

After major improvements of the spinning techniques, of cleaning and etching processes ' and semi-automatic handling of the wafers, IMT was able to produce with high yield wafers with less than $5 \%$ broken anodes. The number of faults was measured by a specially designed electrical test station [8]. If a short was found, the wafer was repaired by etching away the short. Accepted wafers had no shorts and an average of $2 \%$ interrupted anodes.

\subsection{Operation characteristics}

Small size prototypes $10 \times 10 \mathrm{~cm}^{2}$ of MSGCs with coated wafers were systematically tested. The homogeneity of the gas gain over the full area showed variations of less than $20 \%$. During a long series of aging tests, chambers operated with an argon dimethylether mixture of $50 \% \mathrm{Ar}$ and $50 \% \mathrm{DME}$ (Ar-DME 50/50) were illuminated with X-rays over typical areas of up to $113 \mathrm{~mm}^{2}$. The chambers showed constant gain up to charge depositions of $80 \mathrm{mC} / \mathrm{cm}$ corresponding to more than 10 years of HERA-B operation. Rate tests demonstrated that the detectors could be operated at gas gains of 3500 up to rates of $10^{5}$ absorbed X-quanta $/ \mathrm{mm}^{2} / \mathrm{s}$ with a loss of pulse height of less than $40 \%$. In laboratory measurements with a source, efficiencies above 99\% were achieved. This type of MSGCs therefore promised good performance for HERA-B.

In the year 1996 a beam test at PSI ${ }^{5}$ with intense hadron beams was performed to measure absolute efficiencies for MIPs and the spatial resolution in a realistic environment. The test used small size $10 \times 10 \mathrm{~cm}^{2}$ detectors and a pion beam with momentum of $150 \mathrm{MeV} / \mathrm{c}$ which allowed particle fluxes in the beam spot up to 3000 particles $/ \mathrm{mm}^{2} / \mathrm{s}$.

Already the very first day of operation we observed a severe problem which was not encountered in the laboratory. The cathode current showed large spikes, at the same time anodes got lost and sometimes pre-amplifier channels were destroyed. Inspection of the electrode structure in the laboratory under the microscope showed characteristic defects. In the area of the beam spot marks are found on the gold electrodes where the gold is evaporated both from the anode and from the cathode just opposite to each other. A typical photograph is shown in Figure 2. Obviously gas discharges had occurred which sometimes were so intense that they cut an anode strip completely. The lifetime of the chambers in the HERA-B environment with the foreseen technology would have been only months.

$\overline{5}$ Paul Scherrer Institute, Villingen, Switzerland. 


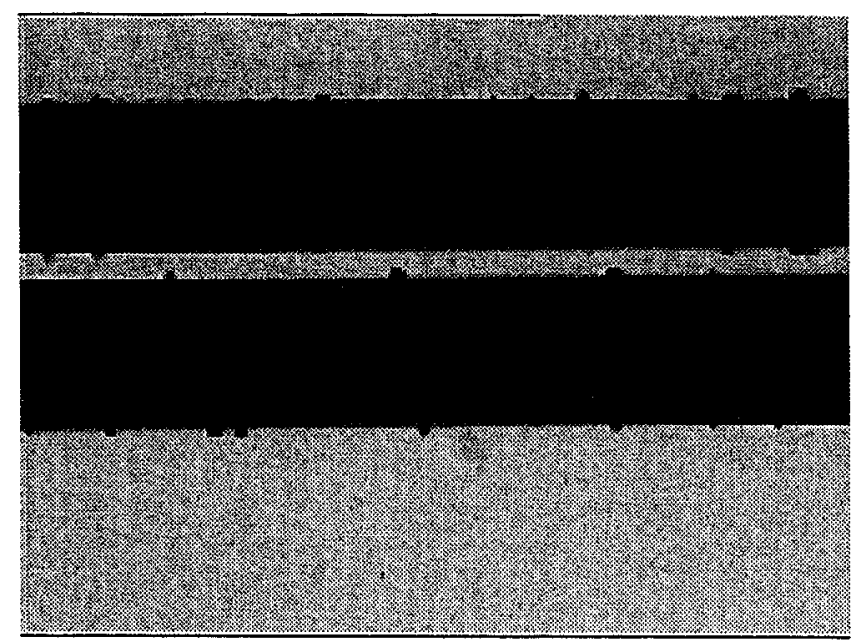

Fig. 2. Photograph of MSGC electrodes after operation of the chamber in a pion beam. Characteristic defects are observed at the electrode borders.

Since discharges had never been observed in intense X-ray beams at exposures with much higher charge deposition rate, it was suspected that the origin of the discharges were strongly ionizing particles like nuclear fragments produced in hadronic beams through interaction with detector material.

A series of laboratory tests where large local ionization was created by particles was carried out to study this effect.

\subsection{Gas discharges by strongly ionizing particles}

The observed sparking phenomena in MSGC and MSGC/GEM detectors have partially been published $[9,10]$. Here a summary of the results is given. Systematic measurements are difficult because the sparking process damages the electrodes and thus modifies the operation conditions during a measurement. Repeating measurements with the same detector often is impossible.

In order to get ionization by heavy particles the counting gas was guided through a stainless steel volume filled with thorium powder. Thorium decays into radium which is transported with the gas into the MSGC gas volume where it emits particles. A typical particle counting rate of $10 \mathrm{~Hz}$ could be achieved this way. The MSGC detectors were operated at nominal voltages and could also be illuminated in addition with an intense X-ray beam. Gas discharges were detected by detection of large induced signals on the cathodes. Alternatively, direct irradiation with a collimated -source through a thin window was used to measure local effects.

A characteristic result from the second method is presented in Figure 3 which shows the spark rate as a function of the cathode and drift voltages measured with a $0.15 \mathrm{~m}$ thick chromium structure and with 1 m thick aluminium electrodes. We observe a strong exponential rise of the spark rate with the cathode voltage which is responsible for the 

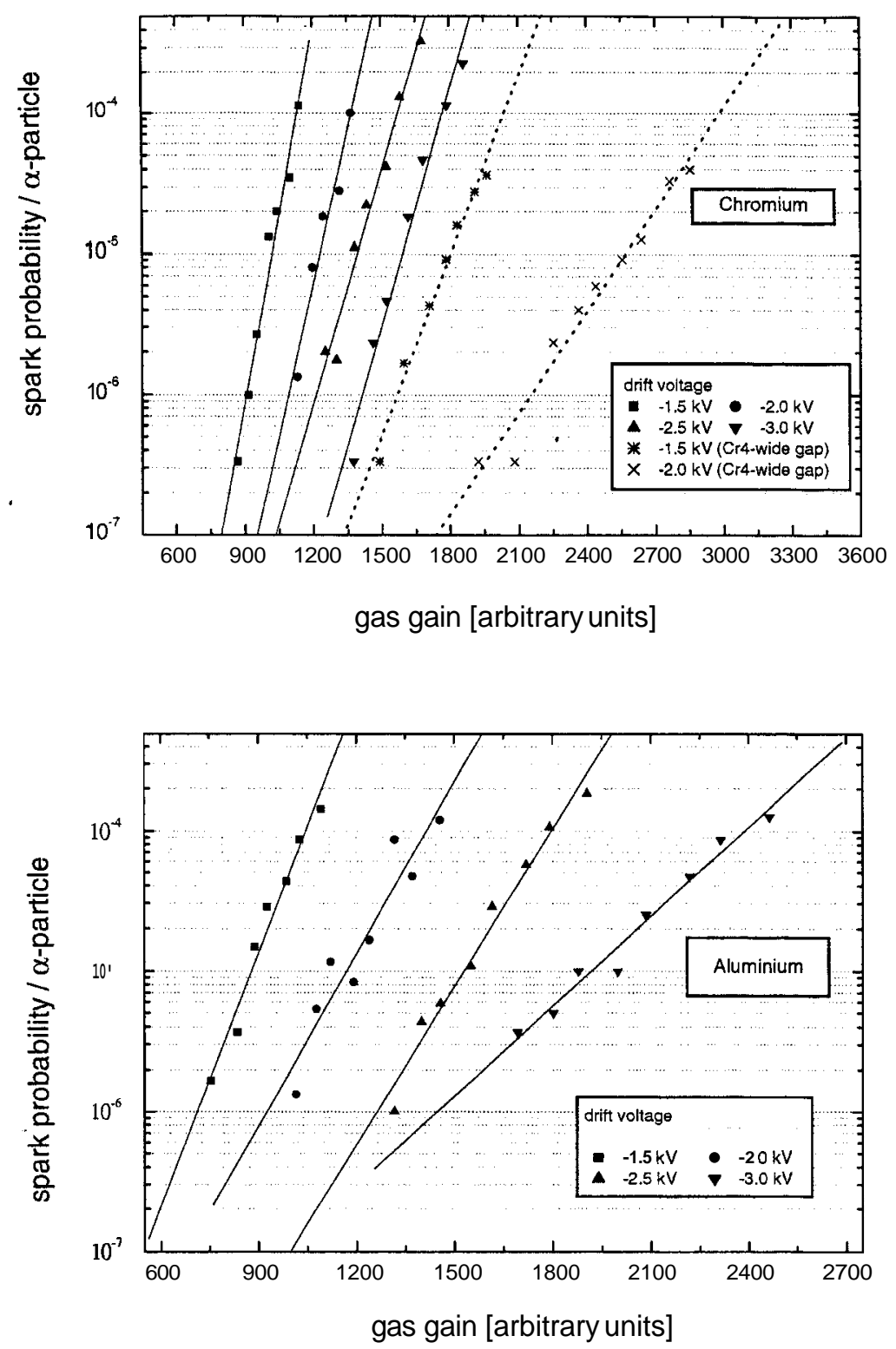

Fig. 3. Spark probability per $\alpha$ particle as a function of the cathode and drift voltages measured with a $0.15 \mu \mathrm{m}$ chromium and a $1 \mu \mathrm{m}$ aluminium structure.

field near the surface and a much weaker dependence on the drift voltage. At fixed gas gain, high drift fields are favorable.

The effect of the width of the anode cathode gap was studied by increasing it by a factor two to $120 \mathrm{~m}$. At fixed gain the spark rate is smaller (see Figure 3), but this positive effect is more than compensated by the more severe damage per spark observed with the wide gap, where the energy stored in the anode-cathode capacitance is much larger.

The high sparking rate of the coated MSGCs is explained by the nearly constant electric 
field component parallel to the surface near the surface, whereas bare glass produces a strongly decreasing field near the anode, which suppresses streamer discharges $[11,12]$. The electric fields between the electrodes computed for a coated and an uncoated substrate are shown in Figure 4. Even though dynamical effects from the space charge created by the avalanche are neglected, the simulation illustrates the qualitative difference between the two configurations.

The explanation for the reduced sparking threshold for coated wafers was confirmed by experimental evidence that sparks were predominantly induced by ionization near the surface and is supported by the favorable effect of high drift fields at fixed gas gain.

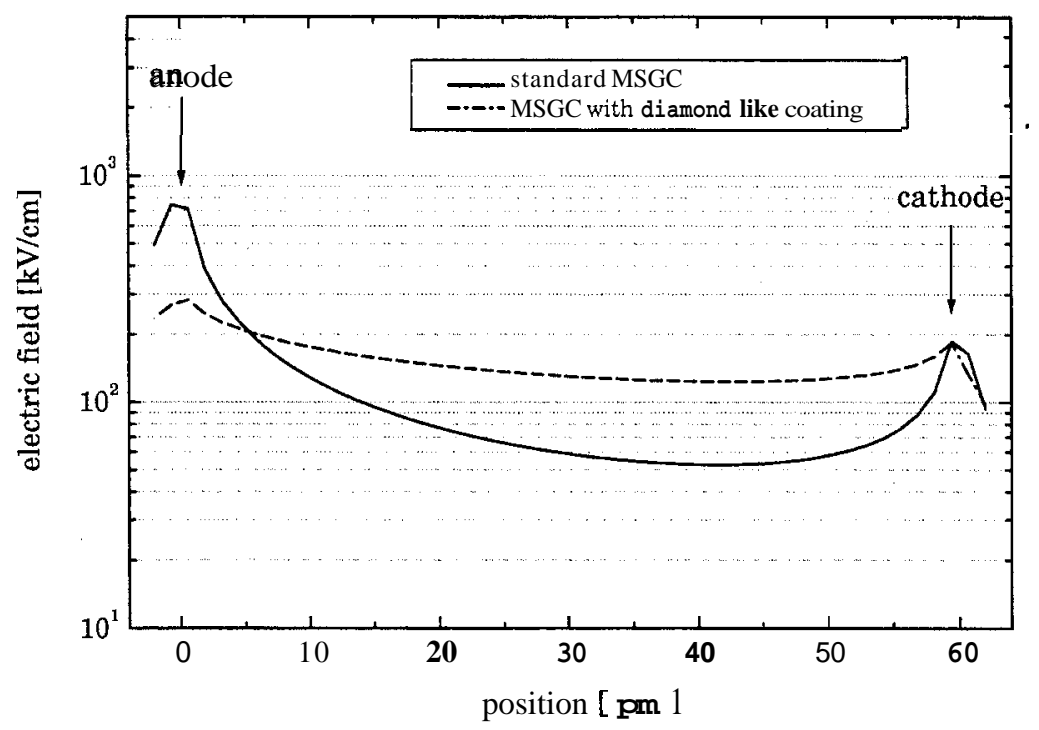

Fig. 4. Simulation of electric eld for coated and uncoated MSGC.

The spark rate should mainly depend on the electric field configuration and the geometry and be rather independent of the electrode material but we expected a different robustness of various electrode metals against sparking. The hope was that elements with high melting points and high binding energies like rhodium or tungsten would be less vulnerable than gold. However, we did not observe any substantial difference between $\mathbf{A u}, \mathrm{Cr}^{6}, \mathrm{~W}$ and $\mathrm{Rh}$ and only a slightly better performance of $\mathrm{Al}$ for a given resistivity of the anode [13]. Aluminium electrodes which can be produced with higher thickness than gold or rhodium sustained a somewhat higher number of sparks before they break but this metal is excluded because of its rather bad aging properties. Anodes with high electric resistivity inhibit discharge of the full anode cathode capacitance in a single spark. They show sequential discharges of low charge. The first spark discharges a few centimeters and produces a local potential drop. A subsequent spark occurs after the potential has again reached a critical value. This process repeats itself until the total charge stored in the anode cathode capacitance drops below a certain minimum. This process limits the total energy release in the discharge and explains the fact that chromium structures can survive more

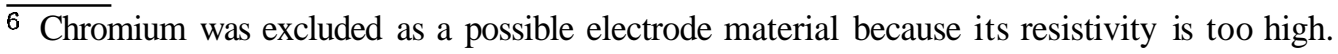
However, it is well suited for systematic studies of discharges in MSGCs. 
than $10^{6}$ sparks without being destroyed. The sequential discharges are well described by simulations of the electric circuitry $[10,13]$.

Unfortunately, as explained in Section 2.2, anodes of high electric resistance are incompatible with the requirement of short readout pulses. The geometry of the HERA-B detectors with long strip lengths and the correspondingly high capacitance per channel of $25 \mathrm{pC}$ (including the contribution from the connections) leads to a white noise of the readout of 2250 electrons per channel. Given our amplifier characteristics, a total gas gain exceeding 4000 is required to get good efficiency. With such a gas gain the discharge rate is unacceptably high in intense hadron beams such that MSGC detectors of the HERA-B geometry cannot be operated.

Ne-DME mixtures were reported to have higher primary ionization [14]. However, our tests have shown that the sparking probability at fixed gain cannot be significantly improved within the uncertainties of the measurement of about $20 \%$.

The sparking problem could only be solved by introducing a gas electron multiplier (GEM) foil [5] as a first amplification step and thereby reducing the gain requirement for the MSGC.

\section{MSGC-GEM detectors}

\subsection{Detector geometry}

The GEMs used for the ITR were produced at the CERN workshop ${ }^{7}$ using a wet etching technique. They were made out of $50 \mathrm{~m}$ thick polyimide (Kapton) foils coated with a $15 \mathrm{~m}$ copper layer on each side. In a final etching step the thickness of the copper layers was reduced to about $7 \mathrm{~m}$. The conical holes have diameters of about $50 \mathrm{~m}$ in the Kapton and $100 \mathrm{~m}$ in the copper. Photographs of the GEM and of its cross section are shown in Figures 5, 6. The holes are arranged in a hexagonal lattice with a hole distance of $140 \mathrm{~m}$ from center to center.

The introduction of the GEM foil required a complete re-design of the chambers. Figure 7 illustrates the construction. The frames which provide the mechanical stability to keep the GEM at constant distance from the MSGC and distribute the gas, consist of two hollow epoxy pieces. The drift gap above the GEM and the transfer gap below are 3.0 $\mathrm{mm}$ and $2.8 \mathrm{~mm}$ wide, respectively.

The geometrical parameters of the detector are summarized in Table 2.

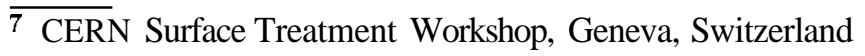




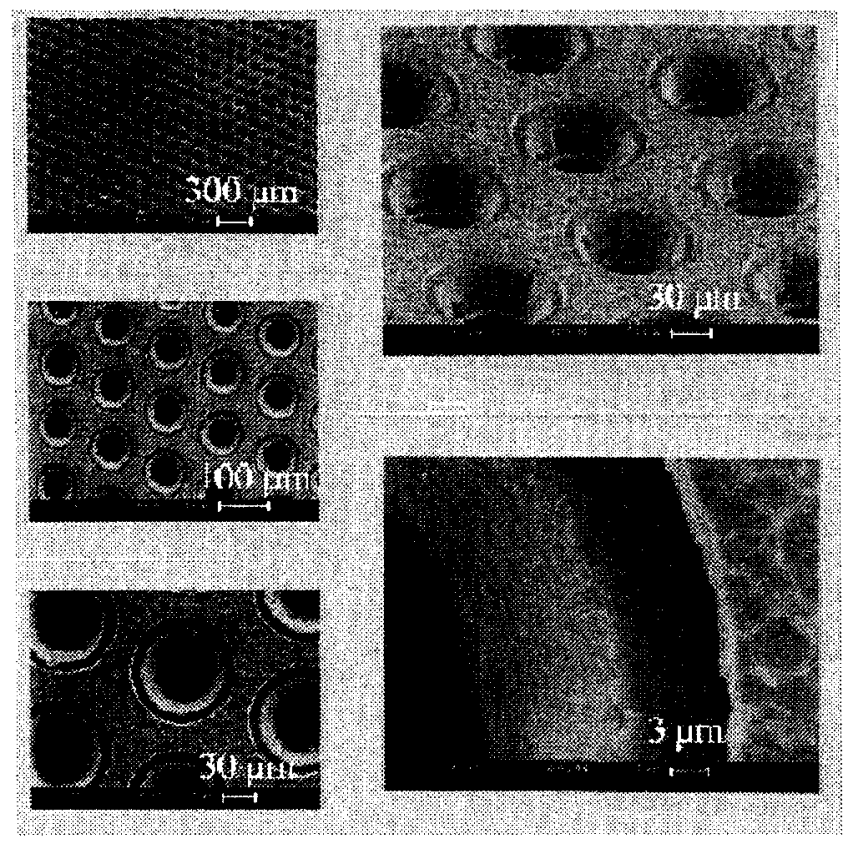

Fig. 5. Photographs of a GEM with di erent enlargements

Table 2

\begin{tabular}{|l|c|}
\hline M S GC & \\
active area, type A & $528 \mathrm{~cm}^{2}$ \\
active area, type B & $589 \mathrm{~cm}^{2}$ \\
wafer thickness & $400 \mu \mathrm{m}$ \\
anode width & $10 \mu \mathrm{m}$ \\
anode cathode gap & $60 \mu \mathrm{m}$ \\
pitch, type A & $300 \mu \mathrm{m}$ \\
pitch type B & $350 \mu \mathrm{m}$ \\
\hline GEM & \\
GEM thickness & $64 \mu \mathrm{m}$ \\
thickness of GEM electrodes & $7 \mu \mathrm{m}$ \\
hole diameter at center & $50 \mu \mathrm{m}$ \\
hole diameter at electrodes & $100 \mu \mathrm{m}$ \\
\hline Frame & \\
height of drift gap & $3 \mathrm{~mm}$ \\
height of transfer gap & $2.8 \mathrm{~mm}$ \\
\hline
\end{tabular}

Summary of the geometrical detector parameters. 


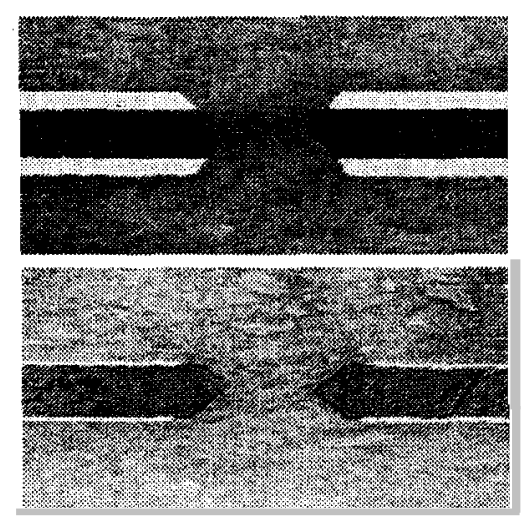

Fig. 6. Cross section of GEM foil before and after nal etching step.

\subsection{Operation characteristics}

Splitting of the gas amplification into two steps allowed to operate the MSGC at moderate voltage. Typical voltage settings during the testing phase were $\mathrm{U}_{\text {cath }}=-520 \mathrm{~V}, \Delta \mathrm{U}_{G E M}=$ $420 \mathrm{~V}, \mathrm{U}_{\text {nr ift }}=2: 5 \mathrm{kV}$ which correspond to gas amplification factors of about 35 for the GEM and 250 for the MSGC. For comparison to reach the same amplification with a single MSGC structure, one would have to apply about $100 \mathrm{~V}$ higher cathode voltages. The detectors were tested intensively in the laboratory and at hadron beams at PSI and in the HERA-B experiment.

The GEM shows the characteristic behavior of devices containing dielectric materials. After switching on the HV, the gas amplification of the GEM rises by a factor of about two within a few hours and stabilizes after a few days, when the polyimide is fully polarized. The gain is rather uniform. It varies by less than $20 \%$ over the full area of the chamber. During irradiation the polyimide charges up and the gain increases by up to $20 \%$. The gain variations with time and rate can be inhibited by coating the GEM foils [15]. Since they are not critical for the operation at HERA we avoided the complication, risk and cost of an additional production step.

With the GEM alone, gas amplification up to $10^{3}$ could be obtained with photon irradiation before sparking started.

A part of the ITR chambers are located inside the spectrometer magnet with a field of $0.85 \mathrm{~T}$. Initial worries that the GEM functionality would suffer in moderate magnetic fields were not confirmed by a test at the nominal HERA-B field perpendicular to the hole axis. No decrease of the gain was observed within $5 \%$ [18].

\subsection{GEM sparks inducing secondary sparks}

First laboratory tests at the PSI pion beam in 1998 of four MSGC/GEM chambers o $p$ erated with Ar-DME 50/50 at a GEM gain of about 50 gave satisfactory results [16]. A second test one year later, however, was similarly disastrous as our first MSGC tests at 

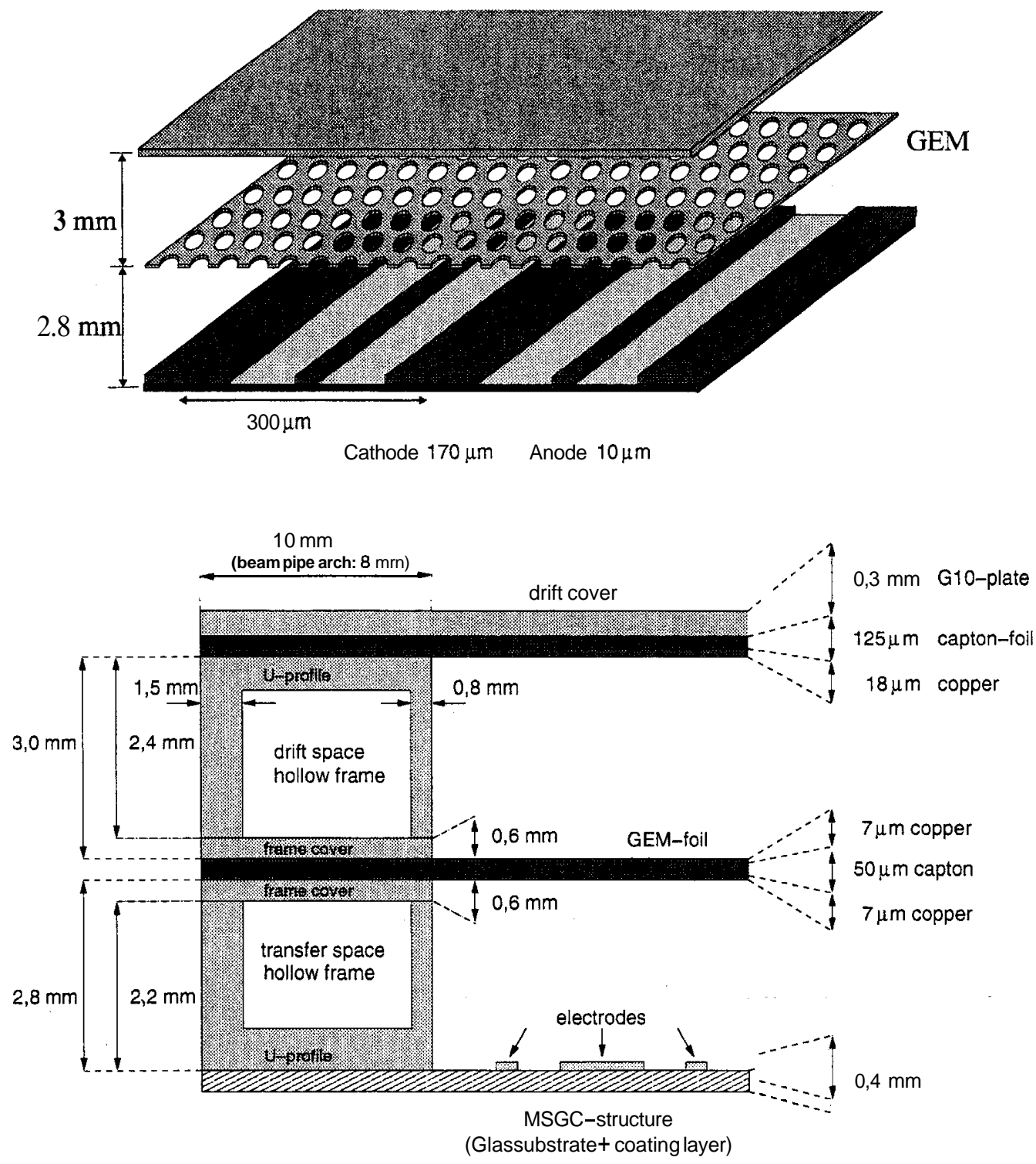

Fig. 7. Schematic view of a MSGC/GEM detector (top) and cross section showing the frame (bottom).

a hadron beam. GEM sparks were observed which induced secondary sparks at the microstrip structure, and sometimes led to discharges between the GEM and the microstrip structure or between GEM and the drift electrode. The electrodes of the MSGC were severely damaged.

In extensive laboratory studies the secondary sparking was reproduced and investigated. The details of the complex sparking process were never fully understood. However, it is obvious that a discharge of the GEM with its high capacitance generates a huge amount of ions and electrons. Even a small fraction of the electrons drifting towards the MSGC, 


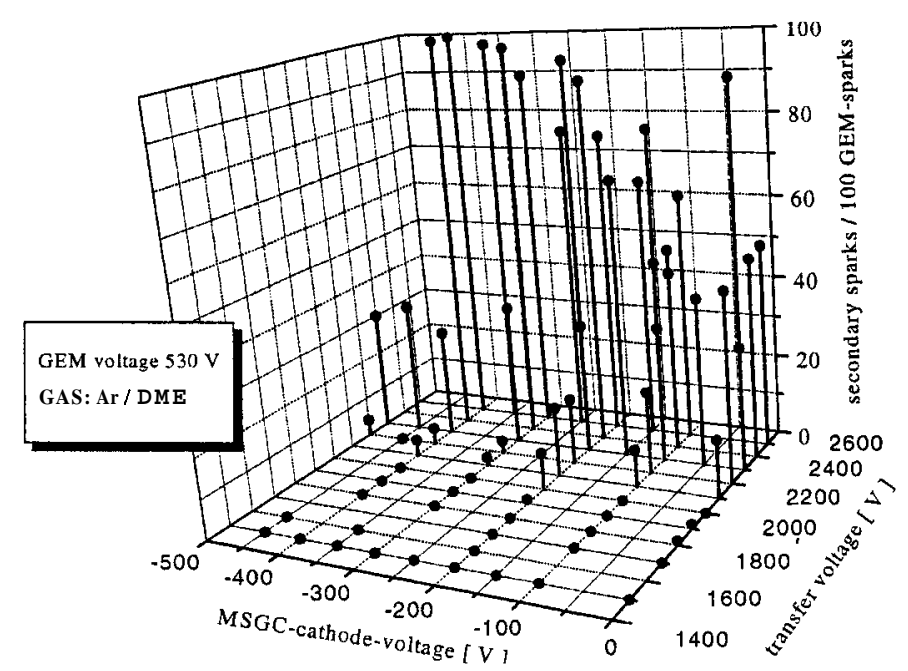

Fig. 8. Spark rate as a function of the cathode voltage and the voltage between GEM and the anodes. A transfer eld of $2.5 \mathrm{kV} / \mathrm{cm}$ corresponds to

with additional gas amplification there, are likely to produce secondary sparks. Thus the rate of those strongly depends on the strength of the transfer field. This was confirmed by laboratory measurements presented in Figure 8 and it explained also the different behavior of the chambers at the two PSI tests which were performed at slightly different HV settings.

A significant dependence of the secondary spark rate on the cathode voltage has not been observed (see Figure 8).

With the voltage between anodes and lower GEM plane limited to below $1250 \mathrm{~V}$ in the HERA-B experiment, the MSGC/GEM detectors were able to cope with the intense hadron flux without suffering from fatal damage by discharges (see Section 5).

\section{Choice of counting gas and aging studies}

Two gas mixtures were systematically studied, Ar-DME 50/50 and $\mathrm{Ar}-\mathrm{CO}_{2} 70 / 30$. The DME mixture has better quenching properties hence less problems with gas discharges. It had also been extensively tested with respect to aging properties by several groups, which consistently reported no aging problems up to large charge depositions on the strips corresponding to many years of LHC or HERA-B operation [17]. Compared to $\mathrm{Ar}-\mathrm{CO}_{2}$ $70 / 30$ it also has larger primary ionization, 24 primary electrons in average for a minimum ionizing particle in the $3 \mathrm{~mm}$ gas gap for Ar-DME compared to 18 for $\mathrm{Ar}-\mathrm{CO}_{2}$ which has also significantly larger transverse diffusion. However, there are also disadvantages of ArDME: The Ar-DME mixture is flammable and constitutes therefore a safety risk. DME is not commercially available with guaranteed purity. Thus every bottle would have to be checked carefully in aging tests to ensure that the detectors are not polluted. In addition, 


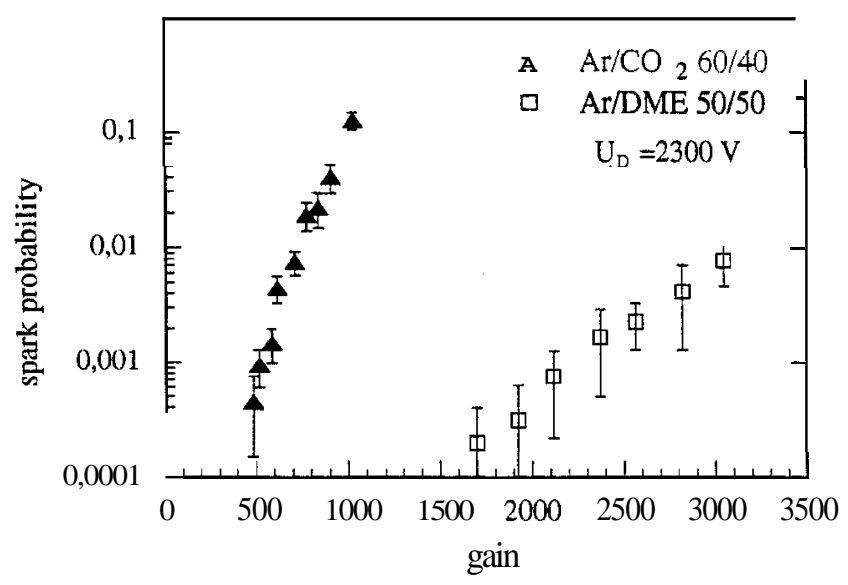

Fig. 9. Discharge rate induced by a-particles in a MSGC versus gas gain (arbitrary units) for two gas mixtures Ar-DME and $\mathrm{Ar}-\mathrm{CO}_{2}$.

we realized that DME is absorbed by Kapton leading to a reduction of the GEM tension with time. After flushing the chamber for 2 weeks, we observed a sag of a foil by $800 \mathrm{~m}$. The original tension could be recovered by changing the gas [18]. The use of DME would have required additional constructional efforts to keep the GEM foil in place.

Comparative sparking tests in the laboratory with particles in the gas indeed demonstrated that the spark protection of $\mathrm{Ar}-\mathrm{CO}_{2}$ is significantly worse for the same gas gain. This is illustrated in Figure 9 which shows the spark rates for $\mathrm{Ar}-\mathrm{CO}_{2}$ and Ar-DME versus gas gain. The result cannot be safely extrapolated to the low operation voltages in a MSGC/GEM detector. It is nevertheless obvious that $\mathrm{Ar}-\mathrm{CO}_{2}$ offers less safety with respect to gas discharges than Ar-DME. Protection against sparking can be strongly improved for $\mathrm{Ar}-\mathrm{CO}_{2}$ by adding small amounts of water to the gas. However, as discussed below, the addition of water led to fast gas aging and was therefore ruled out for HERA-B.

\subsection{Experimental method for gas aging tests}

Big efforts have been made by several groups to study gas aging phenomena in MSGCs [2]. These studies are difficult and hard to reproduce because gas aging depends on a multitude of different parameters. It was however clear from the beginning that the MSGC structures are very vulnerable and that even tiny amounts of pollutions by organic materials can cause fatal deposits on the anodes. Therefore, most of the counting gases which have been used in wire chambers involving organic quenchers cannot be used in MSGCs for high rate applications. Moreover, we made a very careful selection of materials like composites, glues and applied elaborated cleaning and outgassing procedures to avoid pollution of the counting gas. The gas system was entirely based on stainless steel tubing. The sealing rings were made of $\mathrm{Kalrez}^{8}$, materials like Teflon were avoided. All materials were also tested for out-gassing both using gas chromatography and by exposing large surfaces of them to the input stream of counting gas used in gas aging tests of MSGC detectors.

$\overline{8}$ Dupont Dow Elastomers, Belgium. 
An X-ray tube with copper anode was used to produce a high flux of photons. The X-rays were collimated by a lead collimator such that a round area of $113 \mathrm{~mm}^{2}$ was irradiated. The detector was operated at a total gas gain of about 3500. The gas flow was arranged such that the gas in the detector was exchanged twice per hour. The typical counting rate was about $100 \mathrm{kHz} / \mathrm{cm}$ strip length. One X-ray count led to an average avalanche size of about $840000 \mathrm{e}^{-}$and this rate corresponds to a charge deposition rate per $\mathrm{cm}$ strip length which is about 20 times higher than that of the hottest region at HERA-B. This acceleration factor was varied between 1 and 40 for different tests but the large integrated charge depositions with illuminations over several months used acceleration factors of not more than 20. The position of the $\mathrm{Cu} \mathrm{K}_{\alpha}$ line was evaluated in regular intervals during these measurements to track possible changes in the gas gain.

Aging tests were carried out with a large number of detectors over a period of three years. With the full size HERA-B MSGC/GEM pre-series detectors we observed neither for Ar-DME nor for $\mathrm{Ar}-\mathrm{CO}_{2}$ any significant change of the pulse height up to an integrated charge of $45 \mathrm{mC} / \mathrm{cm}$ which corresponds to about 6 years of HERA-B operation. This result was supported by optical inspection of the irradiated areas which showed no sign of depositions on the anodes. This result was interpreted as sufficient reassurance for our choice of counting gas and constructional materials to start mass production of the detectors in spring 1998. It should be noted that our result was in line with similar measurements of other groups [17]. All their results were obtained with small irradiated areas of sometimes only $7 \mathrm{~mm}^{2}$.

\subsection{Observation of gas aging for Ar-DME mixtures}

In autumn 1998 a beam test was carried out at PSI with two full size MSGC/GEM detectors using Ar-DME 50/50 as counting gas. The goal was twofold: during daytime the pion beam was used to study efficiencies, resolution and the performance of our trigger chain. During night time the detectors were exposed to an intense proton beam to accumulate a higher charge dose. The beam spot had a Gaussian profile with a typical full width at half maximum of $5 \mathrm{~cm}$. Within few days a total accumulated charge of $2 \mathrm{mC} / \mathrm{cm}$ was reached in the center region. We observed a rather fast reduction of the drift current during proton irradiation for constant beam current. This was even seen online as shown in Figure 10 for a 12 hour period of proton running at maximal intensity (about 20 times HERA-B charge deposition rate). The detectors were subsequently investigated in the laboratory. Within the illuminated area the detectors still showed acceptable gas gain but outside the illuminated area they did not count at all. Optical inspection showed severe deposits on those anodes which were connected to ground and therefore experienced the nominal electrical field. Disconnected anodes showed no deposits. Depositions were found both in the irradiated area and over the rest of the detector. Parts of an irradiated wafer were sent to the Fraunhofer Institut fur Schicht- und Oberflachentechnik which carried out a surface analysis shown in Figure 11. The anodes in the irradiated area were covered by a layer of $80 \mathrm{~nm}$ pure carbon. A picture of these deposits as seen with an electron microscope is shown in Figure 12. The areas outside, which were not irradiated were covered by an insulating layer of about $35 \mathrm{~nm}$ composed of hydrogen and carbon. 


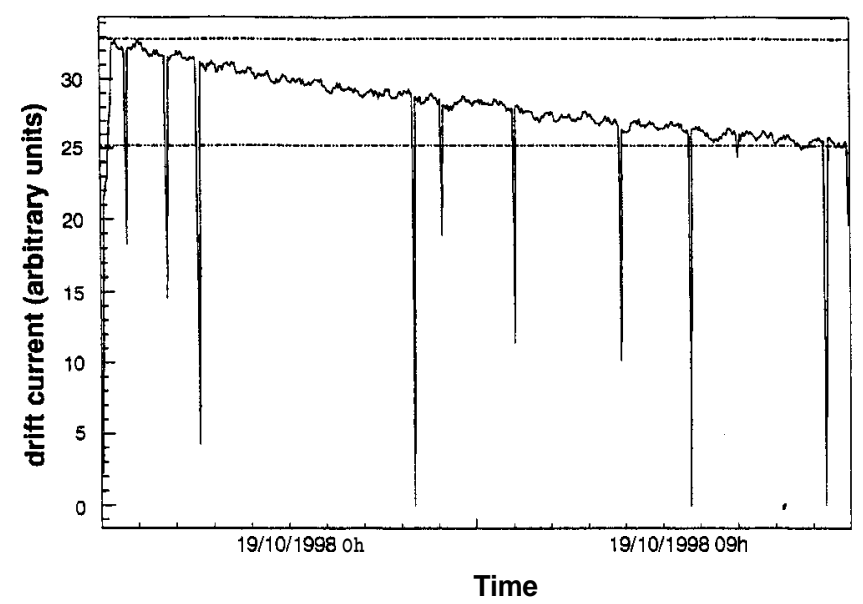

Fig. 10. Drift current (arbitrary units) as measured in the MSGC/ GEM detector versus operation time during intense proton irradiation at PSI. The beam current was kept stable during the exposure.The gaps correspond to beam losses.

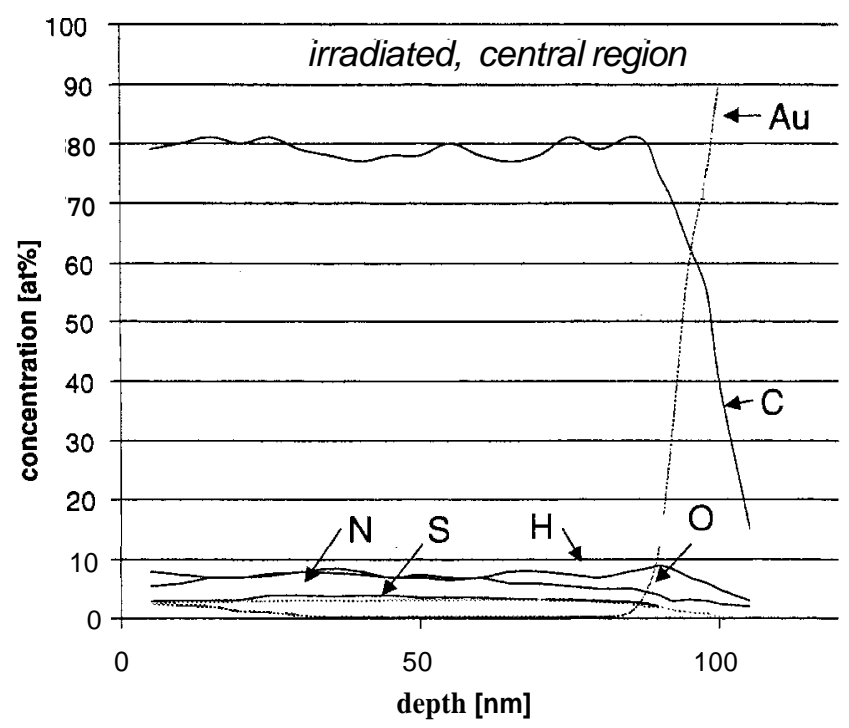

Fig. 11. Concentration of elements as a function of depth for the anode area in the irradiated region. A layer of carbon of $80 \mathrm{~nm}$ thickness is covering the gold surface.

This unexpected result prompted a series of new laboratory aging tests. The final outcome is very surprising. The Ar-DME gas mixture shows gas aging under X-ray irradiation if the area which is irradiated is large enough. This fact is illustrated in Figure 13 which shows the gas gain versus irradiation time for a collimator with an area of $113 \mathrm{~mm}^{2}$ as used before compared to one with an area of $900 \mathrm{~mm}^{2}$. The large area was irradiated at half the local intensity of the smaller one. Identical chambers operated with $\mathrm{Ar}-\mathrm{CO}_{2}$ show no aging independent of the size of the irradiation area.

Apparently, irradiation of a large area with Ar-DME as a counting gas leads to fast aging and visible deposits on the anodes. These deposits are not limited to the irradiated area. 

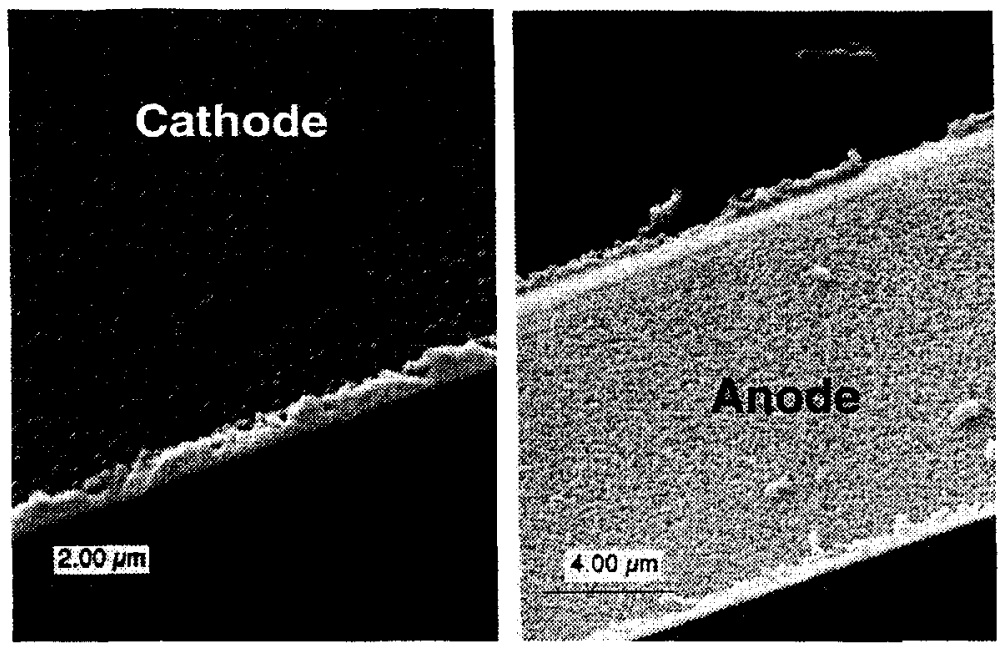

Fig. 12. Photograph (electron microscope) of anodes in the irradiated area of the MSGC after beam test at PSI.

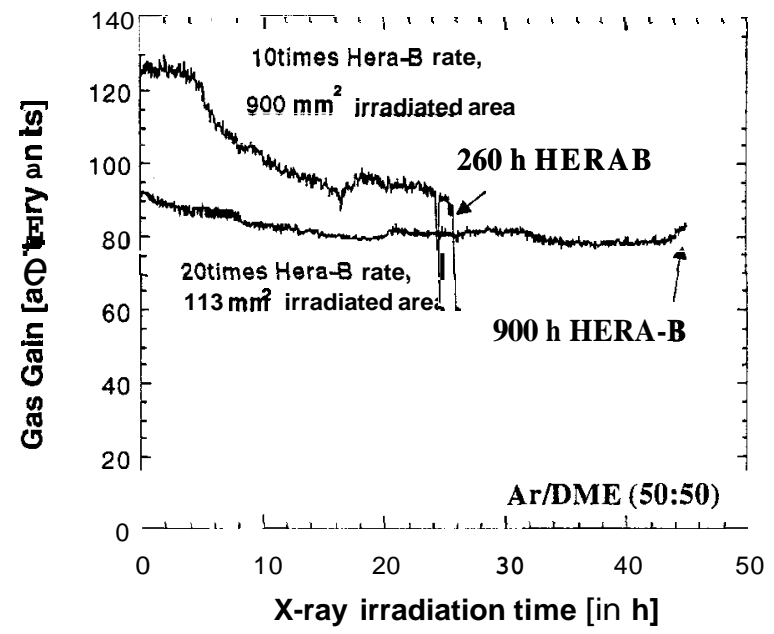

Fig. 13. Gas gain versus irradiation time for a MSGC/GEM detector using Ar-DME 50/50 and two di erent sizes of the irradiated area. The detector was irradiated with X-rays using a charge deposition rate corresponding to 10 respectively 20 times the maximal rate expected at HERA-B .

We therefore have to draw the conclusion that gas aging effects depend strongly on the size of the irradiated area. All evidence supports the assumption that the origin of these deposits is the DME itself. We have no explanation for this experimental fact but it invalidates all aging tests done before.

Subsequently $\mathrm{Ar}-\mathrm{CO}_{2}$ was chosen as the main candidate for the counting gas.

As this gas mixture has low primary ionization and large transverse electron diffusion leading to large cluster width in the detector, we tested several other gas mixtures without hydrocarbons. These were $\mathrm{Ne}-\mathrm{CO}_{2}, \mathrm{Kr}-\mathrm{CO}_{2}$ and $\mathrm{He}-\mathrm{CO}_{2}$ mixtures. The results were un- 


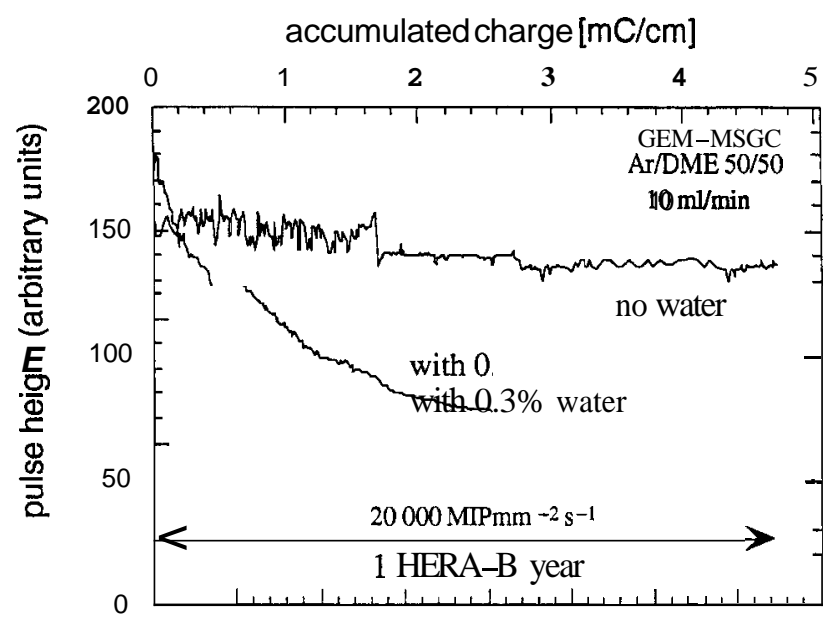

Fig. 14. Development of the pulse height for a gas mixture of Ar-DME with $0.3 \%$ of water and without water as function of the irradiation time.

fortunately discouraging. Measurements with MSGCs showed that for the same avalanche charge on the anodes these gases led to comparable cluster widths but poorer performance with respect to discharge protection. We therefore chose the $\mathrm{Ar}-\mathrm{CO}_{2} 70 / 30$ gas mixture.

\subsection{Aging results for different materials and counting gases}

As explained in Section 3, the safety against gas discharges became a major concern. We therefore tested different combinations of electrode materials and counting gases which promised better protection.

The operation characteristics of a MSGC can be significantly improved by adding small amounts of water, typically $0.3 \%$, to the counting gas. This avoids discharges near insulators e.g. near the detector frames and anode ends and improves the overall protection against sparking, probably by reducing the surface resistivity of plastic materials which tend to absorb water. Addition of water to the counting gas shows however very severe and fast gas aging for both, Ar-DME and $\mathrm{Ar}-\mathrm{CO}_{2}$. This is shown in Figure 14 which compares the pulse height as a function of accumulated charge for Ar-DME with and without an admixture of water. For the gas with water admixture the pulse height drops by a factor three for an accumulated charge of less than $3 \mathrm{mC} / \mathrm{cm}$. Moreover the anodes show deposits in the irradiated area.

We also tested MSGCs with aluminium electrodes. Aluminium electrodes are easier to manufacture and sparking tests showed that the electrodes are more robust against discharges than gold but aluminium is known to introduce aging in MSGCs [19]. Our aging tests with aluminium MSGC/GEM detectors confirmed that this material cannot be used with Ar-DME or with $\mathrm{Ar}-\mathrm{CO}_{2}$. In both cases we observed a fast reduction of the pulse height by more than $20 \%$ for an accumulated charge of only $2.7 \mathrm{mC} / \mathrm{cm}$. Moreover, the 


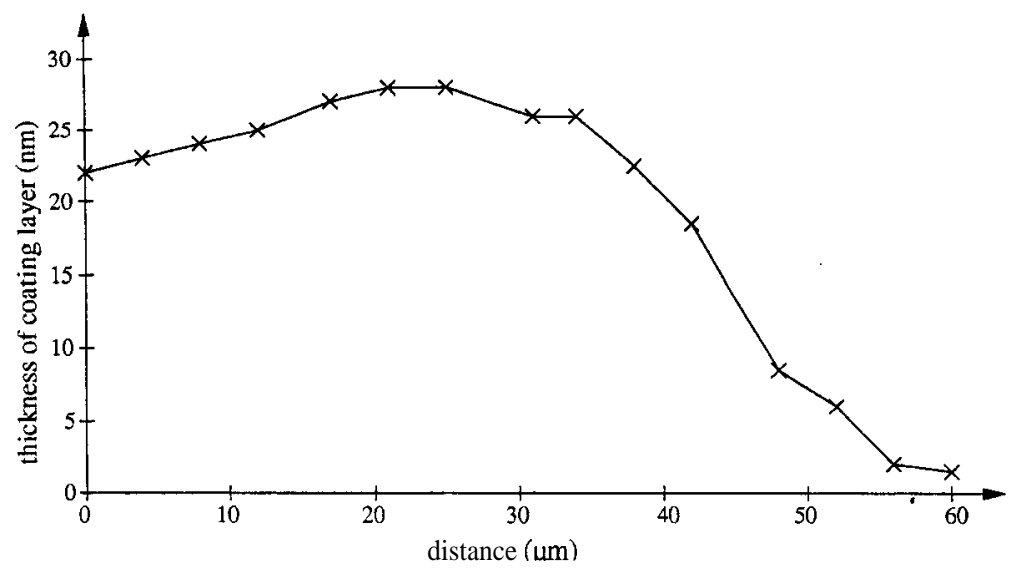

Fig. 15. Thickness of the coating layer across the cathode anode gap after strong irradiation. The anode starts at $60 \mu \mathrm{m}$.

electrodes were severely damaged. Especially the cathodes showed bubbles and craters in the irradiated area.

A rather interesting phenomenon was observed for very long irradiation times for chambers operated with gas mixtures without indication for gas aging like $\mathrm{Ar}-\mathrm{CO}_{2}$ and $\mathrm{Ar}-\mathrm{CO}_{2}-\mathrm{CF}_{4}$ which is used in the Outer Tracker of HERA-B. After a stable period corresponding to about one HERA-B year these chambers show a strong increase of pulse height with time of up to a factor four. For some intermediate time the gas gain becomes very inhomogeneous, changing locally by more than a factor two. After an exposure corresponding to about five years of HERA-B operation the gain becomes stable again but is about four times higher than at the beginning. The gain in this state depends strongly on the counting rate which indicates that the surface near the anodes has become insulating such that charging-up affects the gain. The origin of these effects was clarified by a detailed surface analysis. The intense plasma which is created during the aging tests destroys the DLC layer near the anodes. It is gradually etched away up to the point that there is an insulating strip near the anode edges. This is illustrated in Figure 15 which shows the measured thickness of the coating as a function of the distance between anode and cathode for an area where a charge of $40 \mathrm{mC} / \mathrm{cm}$ was accumulated. The coating is etched away almost completely near the anode and is reduced over the whole gap compared to the starting thickness of about $80 \mathrm{~nm}$. With Ar- $\mathrm{CO}_{2}$ this effect is relatively slow under the conditions of our aging tests, for a gas mixture including $\mathrm{CF}_{4}$ the effect is much faster such that the coating would be destroyed completely for a collected charge of only $2 \mathrm{mC} / \mathrm{cm}$. This gas can therefore not be used. The observed etching effect is of course likely to depend strongly on the local current and plasma density and could be very different for the running conditions of the HERA-B experiment. It is therefore impossible to predict if and how fast such a change would appear at HERA-B. The final beam test at PSI in 1999 using low energy protons was used to accumulate a deposited charge equivalent to 1/3 year of HERA-B operation. During this relatively small exposure no signs of gas aging or damage of the DLC were observed. 


\section{Operation at HERA-B}

\subsection{Treatment of the detector components before installation}

During production, the different parts of the ITR went through several tests:

The wafers were checked at Zurich directly after production (see Section 2.2). Interrupted anodes were recorded.

All GEMs were tested in Heidelberg before chamber construction. The GEMs had to sustain $500 \mathrm{~V}$ in a hitrogen atmosphere for 12 hours.

The individual chambers were transported to Siegen for bonding of the electronics. At Siegen they were flushed with the final chamber gas and put to high voltage. Within a period of 24 hours the voltages were ramped up to $520 \mathrm{~V}$ for the cathode and to $410 \mathrm{~V}$ across the GEM. All currents were recorded and put into a reference file. About half of the chambers showed anode cathode shorts. These shorts were eliminated by disconnecting the anode. In 10 chambers fatal GEM shorts were observed.

Chambers fulfilling the requirements went back to Heidelberg. There the stations were put together and fully assembled stations were shipped to DESY. Before installation another high voltage test similar to the one at Siegen was performed. The required voltages were $490 \mathrm{~V}$ for the cathodes and $400 \mathrm{~V}$ across the GEMs. In 12 out of 144 tested chambers an anode cathode short was detected. One chamber showed a GEM short.

Apparently, each construction or transportation step introduced some damage to part of the detectors and the intermediate high voltage tests could not exclude additional problems occurring later in the hadron beam of HERA-B.

\subsection{Operation conditions and observed problems}

During the period from July 1999 to May 2000 a total of 150 chambers were installed and operated at HERA-B. The first few months of the year 2000 were mainly devoted to commissioning of the detectors. Data were routinely taken from April to the end of the running period by August 2000. The maximum operation time of 1100 hours was reached by 56 chambers. During the last two months $90 \%$ of the installed chambers were routinely participating in data taking without major problems.

The interaction rate at the target varied between $3 \mathrm{MHz}$ and $40 \mathrm{MHz}$. Most of the data were taken at $5 \mathrm{MHz}$. A typical distribution of the hit density in a detector is shown in Figure 16 for $5 \mathrm{MHz}$ interaction rate. The hit density is about 0.006 per anode and event. The shape of the distribution is determined by the beam recess of the MSGC wafer (see Fig. 1) and the radial decrease of particle density.

The raw pulse height distribution cannot be used to determine the gas gain because it is 


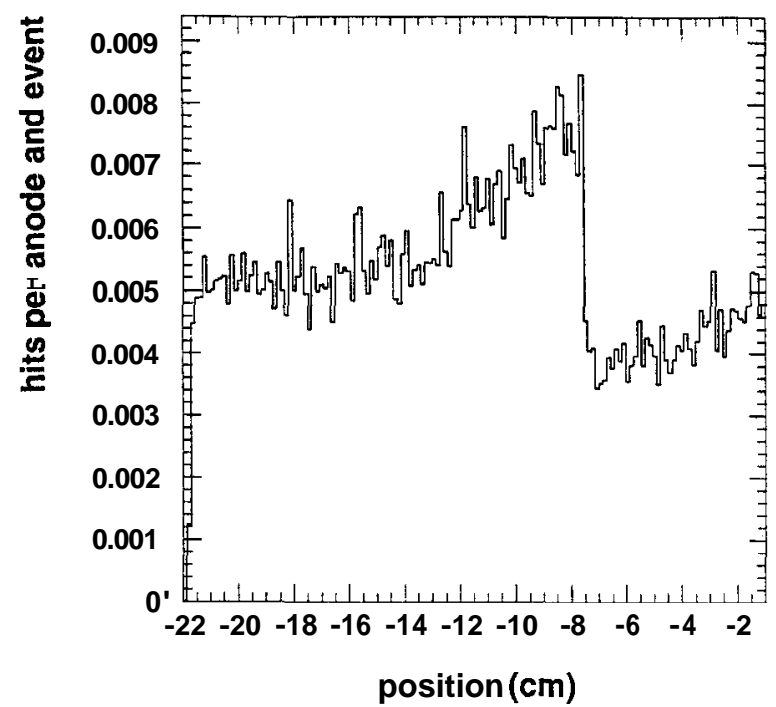

Fig. 16. Hit density as a function of the horizontal distance to the beam axis.

dominated by soft signals which do not belong to tracks. We therefore need to reconstruct tracks first to measure a signal over noise distribution and the efficiency. Since at the beginning of the run the track reconstruction program was not yet well tuned, it was possible only for the chambers in front of the magnet to estimate during running the efficiencies which have to be known in order to adjust the high voltage. Typical voltage settings were $-510 \mathrm{~V}$ for the cathode and 420 to $450 \mathrm{~V}$ for the GEM. Individual GEM voltage settings were necessary to correct for the gain variations of up to a factor 2.5 from GEM to GEM which are caused by slight alignment imperfections of the copper holes at the two sides of the GEM. The high voltage settings for the remaining chambers could not be adjusted and therefore the chambers were operated at GEM voltages below $440 \mathrm{~V}$ and correspondingly more moderate gain.

While at the end of the data taking period running was rather smooth, several problems occurred after installation:

o Four GEMs developed conductive paths between their two copper surfaces. The resistivity dropped to values of the order of MR, values which effectively short the two GEM electrodes.

o In $43 \%$ of the chambers the MSGC electrodes showed one or two short circuits between anode and cathode strips.

These problems occurred shortly after installation and were probably due to spurious dust or production defects.

\subsubsection{GEM sparks}

Sparking in GEMs cannot be avoided completely, but under normal conditions the rate is quite low, of the order of a single spark per day (see Figure 17), a rate which is tolerable. Only six chambers showed sparking at rates above ten per day. 


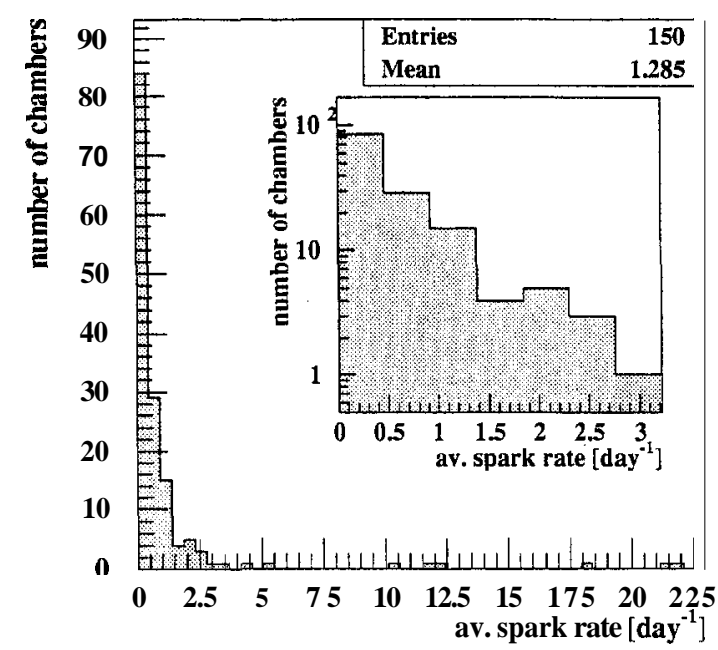

Fig. 17. Number of chambers as a function of their average spark rate. Most chamber show rates below two sparks per day at an average target rate of $5 \mathrm{MHz}$.

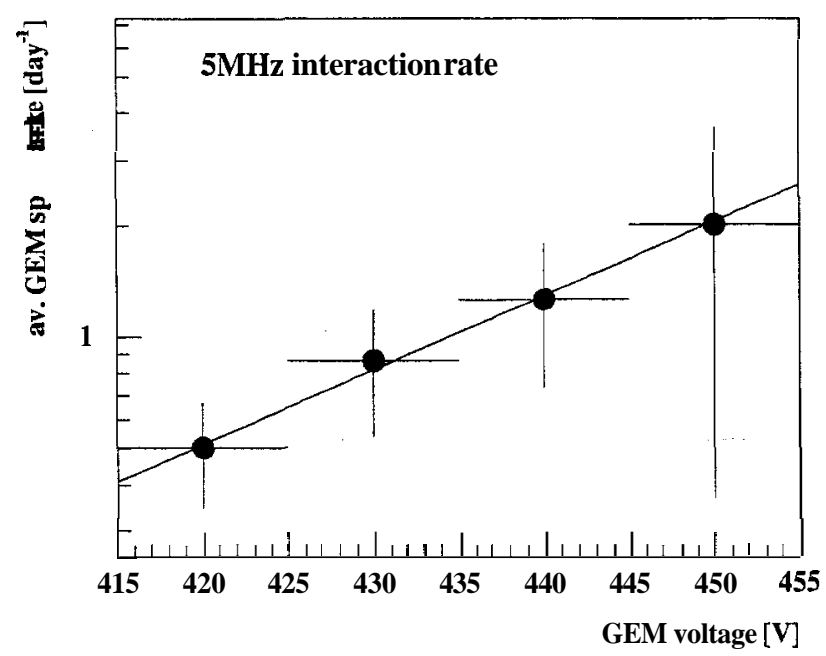

Fig. 18. Spark rate versus GEM voltage averaged over all chambers with rates below six per day. The error bars include systematic uncertainties.

The spark rate increases exponentially with the GEM voltage rising from 1 to 5 sparks/day when the GEM voltage is increased from $400 \mathrm{~V}$ to $450 \mathrm{~V}$. This is shown in Figure 18 for chambers behaving normally. Chambers with exceptional high rates are excluded in this Figure to avoid a biased picture. As expected, the sparking rates increased with the beam intensity. Similar results have been reported in Refs. [20,21] where a linear dependence of the sparking probability on the beam intensity and a strong dependence on the beam composition has been observed.

While under normal conditions the spurious sparking does not disturb the performance of 
the detectors, in some cases continuous sparking produced GEM "shorts" by carbonizing the polyimide surface in the hole.

In some cases when the resulting conductivity across the GEM was low, the "short" could be burned away by applying a short current pulse which evaporates the copper near the affected hole. When this was not possible, the whole chamber 'was lost.

At one occasion, the damage of a GEM could be correlated to a spike in the target rate.

\subsubsection{Anode cathode shorts}

A total of 79 anode cathode shorts were observed during the run period in 2000. Due to the HV grouping of the cathodes this corresponds to a loss of $1.1 \%$ of all anode strips. Out of the total, 29 shorts were produced already within the first 10 hours after switching on and only 16 occurred in the second half of the running period. Most of the late shorts coincided with an increase in the high voltage.

Inspection of the chambers during the HERA luminosity upgrade shut-down revealed that some shorts are correlated to anodes with lithographic defects. Interrupted anodes show a tenfold higher probability to produce a short than perfect anodes. Interrupted anodes have now been disconnected electrically from ground to avoid this problem. The corresponding loss in efficiency is completely negligible.

\subsubsection{Consequences}

Most problems occurred at the beginning of the operation in the beam. They can be avoided or at least reduced to a tolerable level by the following measures:

$\boldsymbol{o}$ operating the detectors for some days at overvoltage before installation,

$o$ carefully'training the chambers in the HERA-B beam before applying the full high voltage,

$\boldsymbol{o}$ HV control based on monitoring of the currents and on spark detection,

$o$ applying a coordinated ramping of the different chamber high voltages,

$\boldsymbol{o}$ avoiding unstable beam conditions.

During the 2000 running period a training procedure has been worked out which has led to a considerable improvement of the reliability of the detectors. The voltages are raised slowly in ten steps over a period of two to four weeks ( 400 hours beam time) depending on the chamber behavior. Very few problems were observed with chambers treated in this way.

A control system based on microprocessors inside the high voltage distribution boxes has been optimized. It continuously monitors the drift and cathode currents and the GEM voltages. In addition, it detects sparking through rapid voltage changes. When a spark occurs, the voltages are decreased thereby avoiding sequential sparks. After some delay, the voltages are automatically ramped up again. The behavior of all chambers is continuously monitored online. 


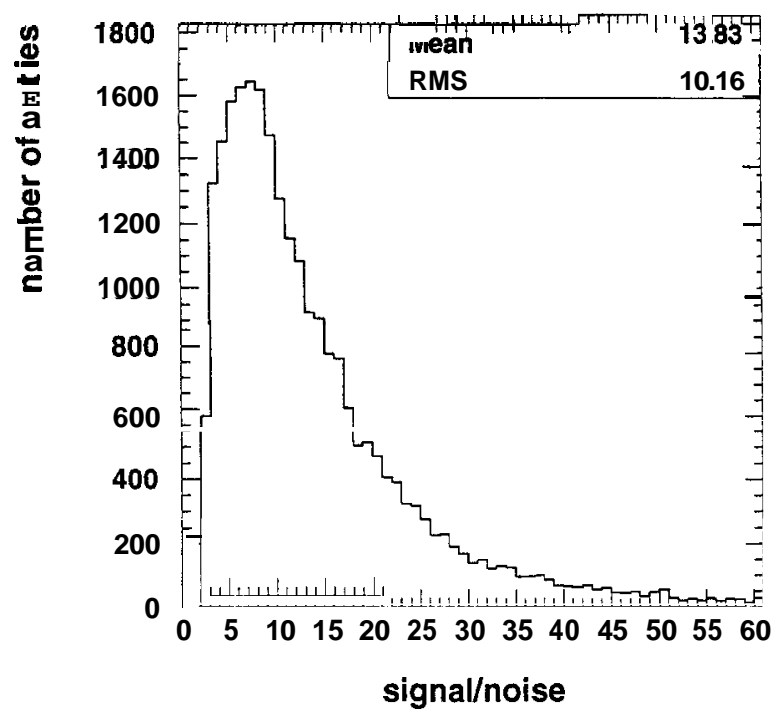

Fig, 19. Signal over noise distribution.

Meanwhile all damaged chambers have been exchanged. Cathode groups have been reactivated by cutting the anode responsible for the short. Extrapolation from the experience gained last year indicates that we should be able to operate the ITR with less than $1 \%$ of channels lost per year.

\subsection{Performance}

The detectors show a noise distribution with a mean value of 2500 electrons. This value is well compatible with the estimate from the strip capacitance and the amplifier input resistor value. A signal over noise $(\mathrm{S} / \mathrm{N})$ plot is shown in Figure 19.

Efficiencies of the chambers in front of the magnet could be determined during running using well reconstructed tracks extrapolated from the vertex detector. They are presented in Figure 20 as a function of the GEM voltage. The efficiencies are above $90 \%$ which is sufficient for tracking.

As mentioned above, there are considerable variations in the GEM performance from chamber to chamber which are compensated by adjusting the GEM high voltage which can be individually set for each chamber. The local gain variations across a single chamber, however, are quite small, namely below $10 \%$.

The strip multiplicity distribution shown in Figure 21 corresponds to a mean value of 2.6 for a threshold of 5000 electrons, which is twice the average noise level. This rather high value is compatible with the large transverse diffusion in $\mathrm{Ar}-\mathrm{CO}_{2}$ along the long drift path of the electrons.

The ITR chambers have been geometrically aligned using an iterative fitting method. A residual distribution for fitted tracks of particles with momenta between 35 and 50 

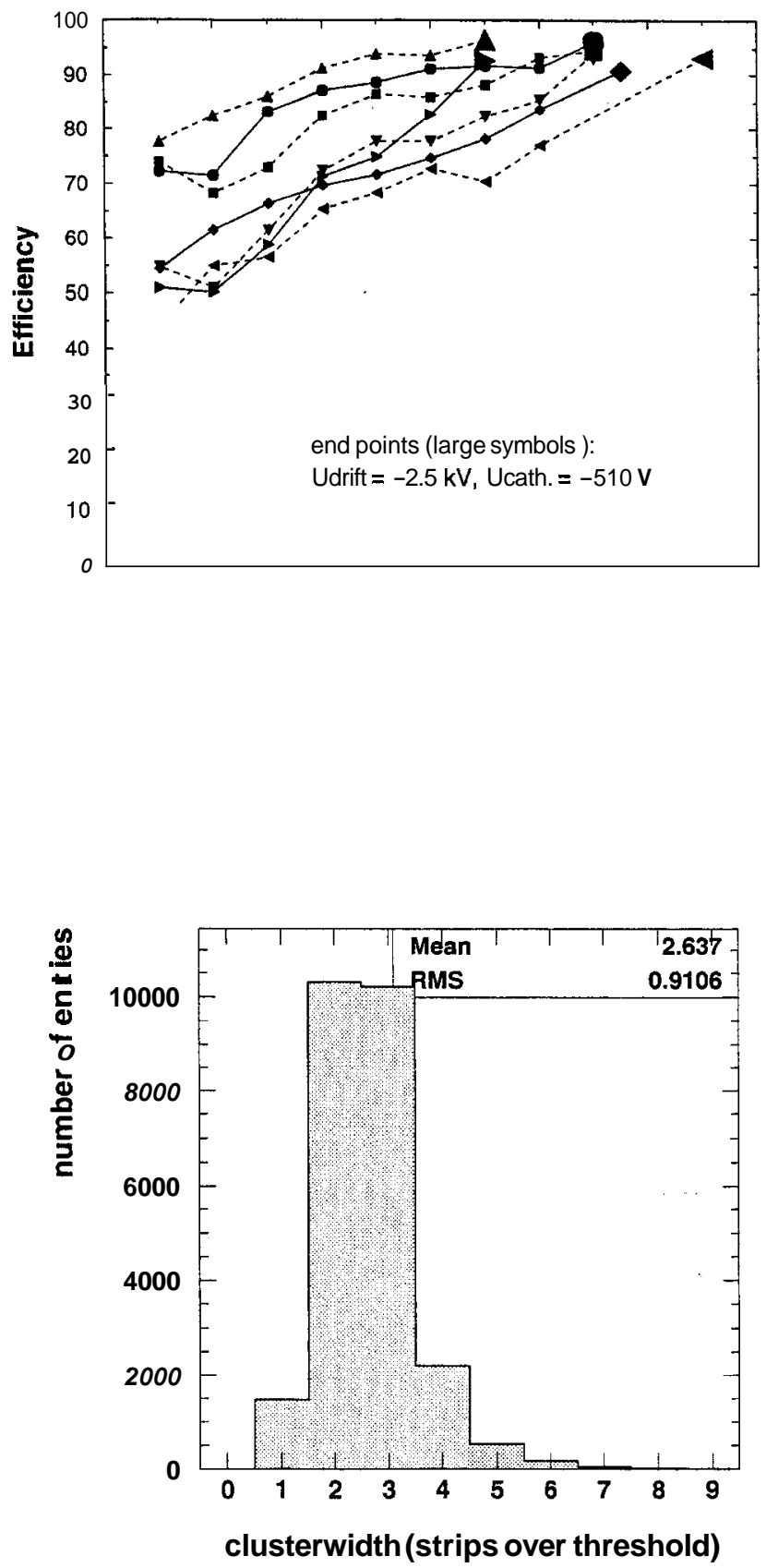

Fig. 21. Distribution of cluster width.

$\mathrm{GeV} / \mathrm{c}$ is presented in Figure 22. In average 15 chambers contribute to one track. From a comparison of the observed root mean error of $105 \mathrm{~m}$ with a Monte Carlo simulation we conclude that the intrinsic spatial resolution of the detectors is better than $110 \mathrm{~m}$. 


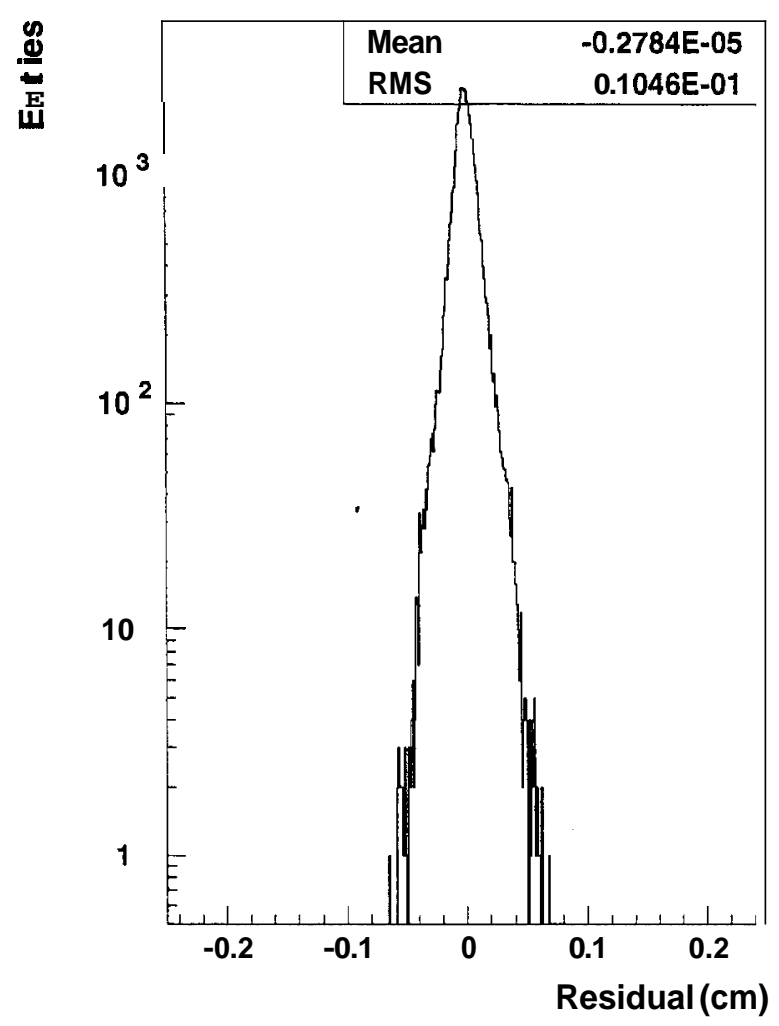

Fig. 22. Distribution of track residuals.

\section{Conclusions}

After many unexpected difficulties the development of the ITR for HERA-B has led to a detector which is working to the required specifications. Efficiencies greater than $90 \%$ were achieved for part of the chambers and are expected for the full detector during the next running period. Extrapolation of the present experience indicates that inefficiencies due to anode cathode shorts will remain below the $1 \%$ level.

Irradiation with X-rays have revealed that aging for Ar-DME gas mixtures depend strongly on the size of the irradiated area. Measurements with $\mathrm{Ar}-\mathrm{CO}_{2}, 70 / 30$ did not show the same behavior and indicate that our detectors would adequately perform for more than six HERA-B years. Irradiation also affects the DLC which is etched away starting from the anode borders. This leads to gain variations with time and beam intensity.

The MSGC with the dimensions necessary at HERA-B and the beam conditions of HERA cannot be operated reliably without the addition of a GEM. At the gas amplification required to obtain sufficient efficiency the spark rates would be lethal. The situation here is different from that at LHC where with much smaller detectors simpler solutions would have been possible [22]. Our system is quite fragile and expensive and requires a complicated HV steering. With the knowledge gained in the last few years, it has become clear that new, more robust devices like multi GEM detectors $[23,20,24]$ are better suited 
for harsh beam environments than MSGC based systems.

\section{Acknowledgment}

We acknowledge the strong effort of the technical stuff of the collaborating institutions. In particular, we wish to thank S. Henneberger, R. Rusniak, A. Rausch, Ch. Rummel, D. Gieser, R. Eitel and S. Rabenecker (Heidelberg), O. Meyer, G. Schmidt (Siegen), K. Boesiger, K. Esslinger, B. Schmid and S. Steiner (Zuerich), Serguei Cheviakhov (DESY). Our work profited very much from the close collaboration with the vertex detector group of the MPI Heidelberg, especially in the areas of readout and monitoring.

\section{References}

[1] T. Lohse et al., "An Experiment to Study CP Violation in the B System Using an Internal Target at the HERA Proton Ring", DESY-PRC 94/02 (1994).

[2] F. Sauli et al., "Development of Micro-Strip Gas Chambers for Radiation Detection and Tracking at High Rates", Status report, CERN/ DRDC/ 93-34, (1993).

[3] F. Sauli et al., "Development of Micro-Strip Gas Chambers for Radiation Detection and Tracking at High Rates", Status report, CERN/ DRDC/ 94-45 (1995).

[4] CMS-Collaboration, "Status report and milestones", CERN/ LHCC 94-20 (1994).

[5] F. Sauli, "GEM: A new concept for electron gas ampli cation in gas detectors", Nucl. Instr Meth. A 386 (1997) 531.

[6] S. B. Visbeck, "Untersuchungen von Prototypen der Mikrostreifen Gaskammern (MSGC) des inneren Spurkammersystems des HERA-B Experiments", diploma thesis, Heidelberg (1996).

[7] E. Ermert et al., "Study of electric properties of microstrip gas counters with and without coating", in Proc. 3rd Int. Workshop on Micro-Strip Gas Chambers, Lyon, 1995.

[8] Thomas M. Walter, "Contributions to the Development of Microstrip Gas Chambers (MSGC) for the HERA-B Experiment", Ph.D. thesis, Universitat Zurich 2001.

[9] B. Schmidt, "Microstrip gas chambers: Recent developments, radiation damage and longterm behavior", Nucl. Instr. Meth. A 419 (1998) 230.

[10] S. Keller et al.,"Sparks in MSGCs", Nucl. Instr. Meth. A 419 (1998) 382.

[11] V. Peskov et al., "Feedback and breakdown in microstrip gas counters", Nucl. Instr. Meth. A 392 (1997) 89.

[12] A. Bressan et al., "High rate behavior and discharge limits in micro-pattern detectors", Nucl. Instr. Meth. A 424 (1999) 321.

[13] S. Keller, "Funkenuberschlage in Mikrostreifengasdetektoren", diploma thesis Siegen (1998)

[14] G. Bayatian et al., "The Tracker Project", CMS TDR 5, CERN/ LHCC 98-6 (1998). 
[15] S. Beirle et al., "Carbon coated gas electron multipliers", Nucl. Instr. Meth. A 423 (1999) 297.

[16] H.-B. Dreis et al., "Operation of a large GEM-MSGC detector in a high intensity hadronic test beam using fully pipelined readout electronics", LHCb note TRAC-98/ 060.

[17] R. Bouclier et al., "Performance of Gas Microstrip Chambers on Glass Substrates with Electronic Conductivity", Nucl. Instr. Meth. A 332 (1993) 100.

[18] M. Hildebrandt, "Entwicklung und Bau der Detektoren fur das Innere Spurkammersystem bei HERA-B", Ph.D. thesis, Heidelberg (1999).

[19] Bateman et al., "The Experimental Characterisation of Gas Microstrip Detectors, III Lifetime Characteristics", RAL Report, RAL-94-114 (1994).

Bateman et al., "Rate and lifetime characteristics of a gas microstrip detector fabricated on sputtered S8900 glass", RAL-TR-95-032 (1995).

[20] M. Ziegler et al., "A triple GEM detector with two-dimensional readout", Nucl. Instr. Meth. A 471 (2000) 260.

[21] S. Bachmann et al., "Performance of GEM detectors in high intensity particle beams", Nucl. Instr. Meth. A 470 (2001) 561.

[22] V. Zhukov for CMS Collaboration, "Large scale test of MSGC + GEM detectors in a high intensive hadron beam", Nucl. Instr. Meth. A 461 (2001) 118.

[23] Bachmann et al., "Charge ampli cation and transfer processes in the gas electron multiplier", Nucl. Instr. Meth. A 438 (1999) 376.

[24] B. Ketzer et al., "GEM detectors for Compass", IEEE Nucl. Sci. Trans. NS-48 (2001) 1065. 


\title{
Aging of Gaseous Detectors: assembly materials and procedures
}

\author{
M. Capeans*
}

CERN

\begin{abstract}
Aging of gaseous detectors is known as the degradation of their performance under the exposure to ionizing radiation. It is a complex phenomenon that depends on many parameters. Among others, aging depends on the gas mixture and may be enhanced by the presence of pollutants in the gas. The origin of the impurities is diverse and includes outgassing from assembly materials and the gas system components, and contamination of the detector during the assembly process. Systematic studies on this topic have been carried out. Methods used to ascertain the outgassing properties of materials are described and compared. Materials that might be used for assembling gaseous detectors and associated gas systems are catalogued according to their outgassing rate. Some factors affecting the aging rate in some fast gases are presented. Finally, a set of recommendations to build and operate gaseous detectors in high luminosity experiments is given.
\end{abstract}

\section{Aging of gaseous detectors}

Gaseous detectors have been used for many decades in modern high energy physics experiments for particle detection and tracking. Soon after their development, it has been common to find problems associated with their long-term exposure to radiation, limiting their useful time. The observed phenomenology included the appearance of local and permanent damages detected as self-sustained discharges, excessive currents, gradual loss of energy resolution and decrease and non-uniformity of the gas gain. These observations led to the association of the problem to the presence of layers on the electrodes, possibly induced by pollution released by materials used in the gas system or chamber construction, or impurities in the gas itself. A branch of Chemistry called Plasma Chemistry studies processes with certain qualitative analogies to those that take place in the plasma surrounding the anodic wire in a gaseous detector, which might be considered as a dc plasma at 1 atmosphere. Plasma is a mixture, initially overall neutral, of positive and negative ions, neutral species, and chemically active free radicals; radicals can recombine back to the origin molecules or new ones. Polymerization is thus the process by which some monomer radicals associate in subsequent chain reactions to form a very large molecule, frequently of high molecular weight. Created polymers are solid, highly branched and cross-linked, have excellent adhesion to surfaces, are resistant to most chemicals and insoluble in most solvents. Deposits observed in aged

*mar.capeans@cern.ch

CERN, EP div., CH-1211 Geneva 23 
chambers seem to have similar properties. During operation of a gaseous detector, formed molecules will be removed by the gas flow if they are light enough, or be deposited or react with electrode materials inducing typical aging processes. The chemistry of the process has not been deeply studied; thus a rigorous explanation of why certain chambers age and others do not cannot be yet discussed with certainty. In addition, information gathered form experiments, as distinct from controlled tests in laboratory, is sometimes contradictory and renders extrapolation to other cases difficult. The approach to the aging problem is still in most cases purely experimental. A summary of principal results until 1986 may be found in the Proceedings of the first Workshop on Radiation Damage to Wire Chambers [1]. Since then, aging has been extensively studied and even more in the last decade in view of the imminent high luminosity experiments $[2,3,4,5]$. Observations are difficult to compare and/or extrapolate between one another. This work compiles existing data and systematic observations with the aim of determining a set of operating conditions, such as gas choice, purity and cleanliness of the system, detector assembly materials and procedures, additives and level of impurities under which gaseous detectors do not age up to reasonable levels of radiation.

\section{Contributions to the aging process}

The mechanisms leading to aging of gaseous detectors can be summarized as follows:

- Polymer formation in the avalanche plasma from the cloud of ions and radicals of the gas filling. This is the case of hydrocarbon molecules used in detectors exposed to large particle fluxes. Pollutant molecules may trigger or accelerate the polymerization process for a given gas mixture.

- Direct deposition on the surface of electrodes and insulators of pollutants, which are transported with the gas flow and deposited thanks to electrostatic forces. Their origin is diverse: i) outgassed vapours from organic materials. ii) Some reactive gases can be incompatible with chamber materials and plumbing. Dymethyl ether is a sample case. iii) Materials might also follow structural changes induced by the effect of ionizing radiation; new outgassed pollutants may promote polymerization.

- Some initially neutral gases become reactive because of the species created around the wire in the avalanche process. The created species are reactive enough to remove concurrently layers of some materials, mechanically damaging the detectors and polluting the filling gas. Tetra fluoride radicals are capable, for example, of removing thin layers of deposits on coated wires. This capability might on the other hand turn into a serious problem when the active species attack assembly materials. 


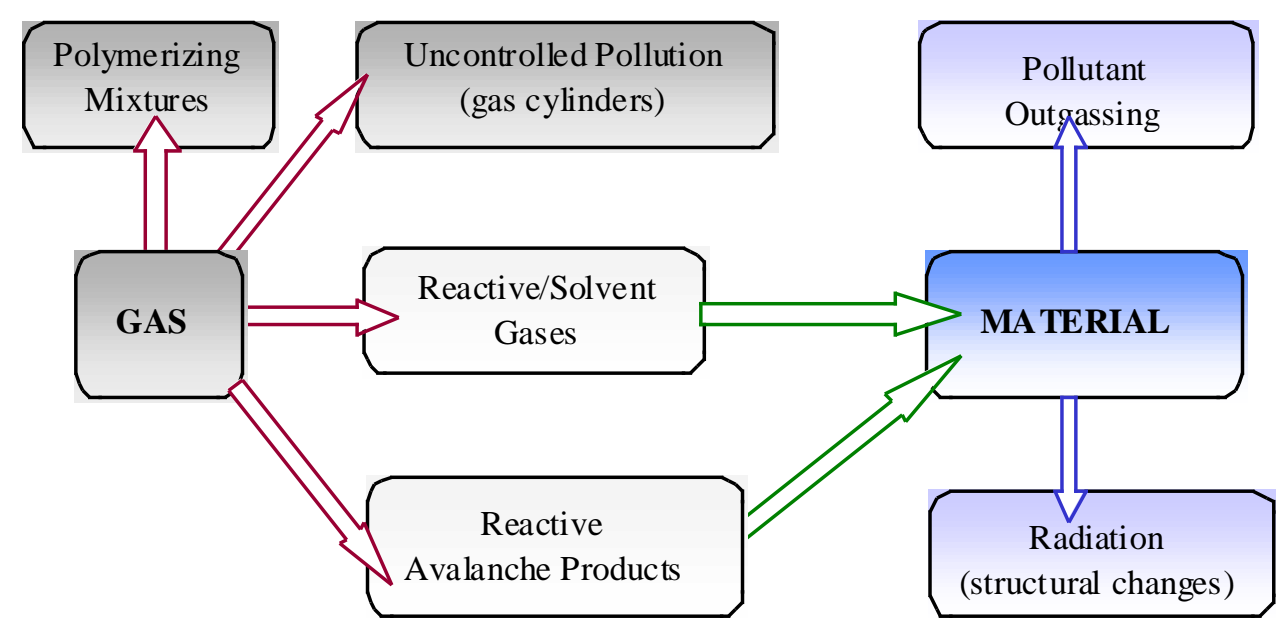

Fig. 1 Contribution of materials and gas to the aging processes.

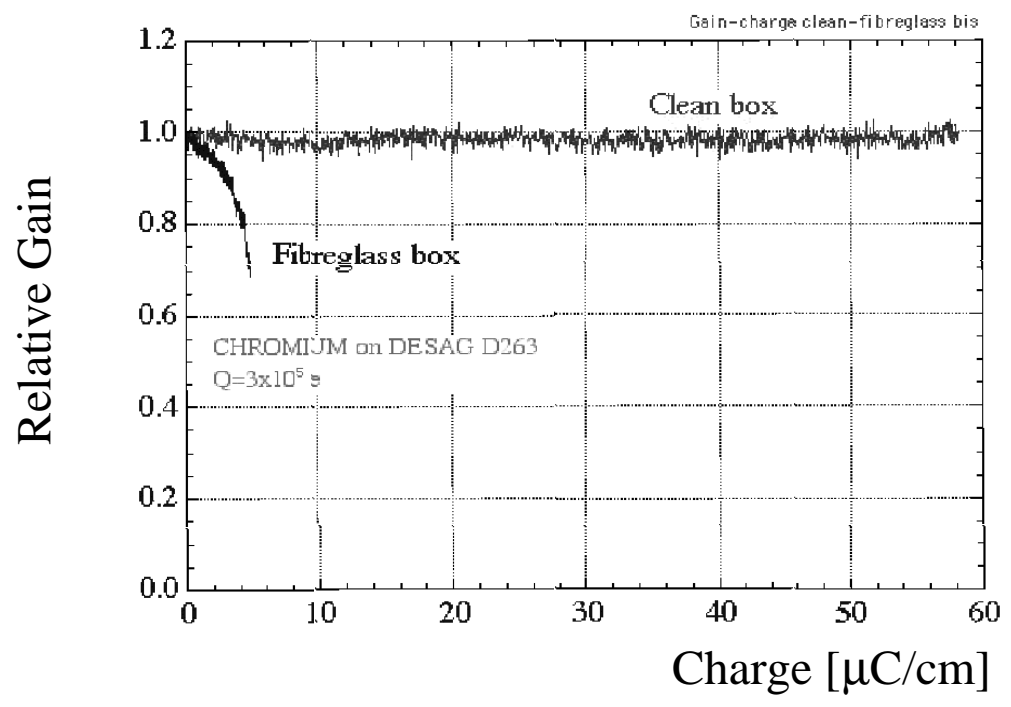

Fig. 2 Aging rate measured for two identical MSGC plates (Cr strips on DESAG 263) assembled with different materials. Very rapid aging is measured for the plate assembled with conventional MWPC materials, such as fibreglass, Araldite 106 epoxy and rubber O-rings.

The probability of all these mechanisms to occur depends on a large number of factors. Gas flow, gas gain, the geometry and electric field configuration, presence of additives and others play an important role. The contribution of materials and gas pollution to trigger aging processes is shown schematically in Fig. 1. The importance of materials selection is manifested in Figs. 2 and 3. Fig. 2 [6] shows the dramatic effect of the choice of materials used to assemble Micro Strip Gas Chambers (MSGC). The rate of aging increases orders of magnitude simply by using some organic elements in the assembly, a conventional choice for low rate multiwire proportional chambers. Fig. 3 [4] shows different aging rates measured with the same MSGC connected to two different gas systems: the ultra clean system is 
assembled and equipped with components following ultra high vacuum standards (VCR connections, welded electropolish stainless steel piping, long metallic exhaust pipe, etc) and the minimal quantity of possibly polluting components (one Viton and one Kalrez joints in the electronic mass flowmeters); the second gas system is assembled with the minimal possible sources of pollution, but standard metal valves and few rubber O-rings and sealants.

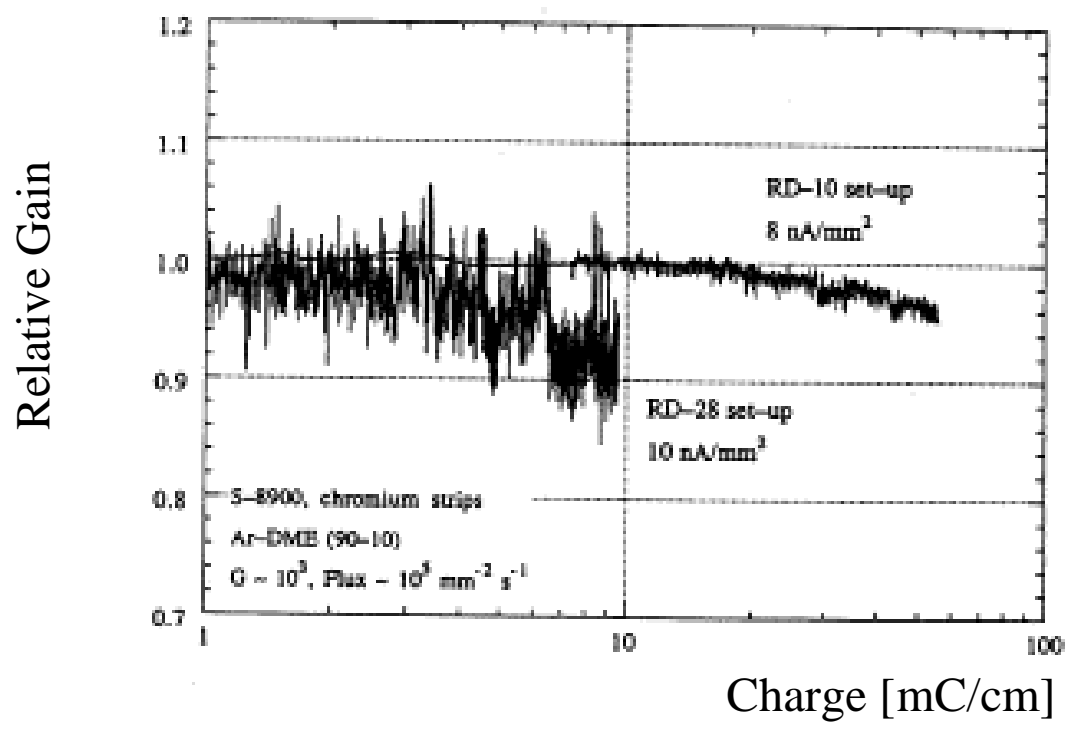

Fig. 3 Gain dependence on charge measured with the same MSGC operated in a ultra clean gas system and a clean system containing few rubber O-rings, valves with Teflon joints and few spots sealed with Torr-Seal.

A systematic search aimed to find possible pollutants in the standard set-up identified few ppm of $\mathrm{C}_{2} \mathrm{Cl}_{4} \mathrm{~F}_{2}$ released at each manoeuvring of the metal valves as capable of significantly accelerating the aging rate of the MSGC [7]. The source of pollution could be the Teflon joint, some lubricant or any residual cleaning product used during the assembly of the valve. Once the source of pollution was identified, all valves were removed from the gas mixer and cleaned just by baking them at around $100{ }^{\circ} \mathrm{C}$ for few hours. Further aging tests carried out in this setup confirmed the excellent degree of cleanliness.

By suitably choosing the operating conditions, gaseous detectors work satisfactorily up to collected charges equivalent to many years running in high luminosity experiments. We will show that an adequate choice of materials for detectors and gas systems and assembly procedures seems mandatory to minimize aging effects.

\section{Outgassing tests}

The use of new materials with attractive mechanical, electrical and radiation resistance properties is conditioned by their outgassing properties when in contact with a given mixture. We describe in this section some methods used to determine the rate of outgassing of some assembly materials and parts for gas systems. 


\subsection{Gas analysis}

A method to determine the outgassing properties of certain material is the analysis of a gas sample that is in contact with the material. A basic test consists of flushing clean gas through a box where the material under investigation has been introduced and analyzing the gas as it flows out. The surface of each individual sample and the gas flow should be kept constant to allow comparative studies. The material can be heated up in order to increase the rate of possible outgassing, so that relative large amounts of pollutants can be produced in a few days, thus increasing the sensitivity of the measurements (unfortunately the scaling factor is unknown). Still impurity detection has the intrinsic limitations of the analysis station: i) the incapacity to detect substances below some given quantity, and ii) the inability to separate, thus identify, certain compounds. Another drawback is that even if outgassing is detected, the particular pollutant might not be harmful for the gaseous detector operated at high rate.

Data presented here has been obtained analyzing the gas composition and possible pollutants using a Gas Chromatograph ${ }^{1}$ (GC) and two associated detectors: a Mass Spectrometer ${ }^{2}$ (MS) and an Electron Capture Device ${ }^{3}$ (ECD). The GC is simply an oven with a capillary column capable of separating gas substances depending on their interaction properties with the column. A signal appears for each separated compound at some retention time, defined by the column and temperature profile. In the MS detector a $70 \mathrm{eV}$ electron source ionizes and fragments molecules. A quadrupole mass filter sorts the resulting ions according to their mass over charge $(\mathrm{M} / \mathrm{Z})$ ratio. Signal appears as ion abundance as function of retention time in the column or $\mathrm{M} / \mathrm{Z}$ ratio. As a result, identification of each molecular compound is possible, having detection sensitivity up to the ppm level. The ECD is the second detector connected to the GC. Its operation is based on the fact that at normal temperature and pressure some gases behave as perfect insulators. Consequently, the presence of very few charged species (electronegative molecules such as halogens and halocarbons -freons-) can be readily observed by amplification in an electric field. There is no information other than signal amplitude versus retention time, and a specific calibration has to be made for each compound to attain pollutant identification. Its advantage is the extreme detection sensitivity, better than ppb.

\subsection{Gas analysis and aging test}

A positive result obtained with the method described in the previous section should be considered as necessary although not sufficient for a material to be used for assembling a detector. The definite test should consist of an aging test. Comparable from a qualitative point of view is the answer obtained monitoring the gas gain of a clean, validated gaseous detector, which is connected downstream the outgassing box where the material under test is introduced, and strongly irradiated. This test allows correlating the presence of impurities in the gas with aging effects in the detector. In this case the response is more extensive than the

\footnotetext{
${ }^{1}$ Hewlett Packard 5890 Series II

${ }^{2}$ Hewlett Packard Mass Selective Device 5971 A

${ }^{3}$ Hewlett Packard G1223 A
} 
simple gas analysis, but unfortunately the irradiation conditions (high dose rates) make it difficult to extrapolate the results to the final running conditions and lifetime scale of the experiments. Thus existing data obtained either from systematic outgassing studies or experience gained with detectors has only a pre-selective character when designing new detectors.

\subsection{TML and CVCM tests}

A quick method to search for low outgassing materials was developed by NASA in the early 60s. The method consists of testing micro-quantities by condensing the volatile products to determine the amount of volatile condensable materials. This method gives a much faster answer than the detailed analysis of gas samples, where specific quantitative and qualitative calibrations have to be done to determine each detected pollutant and its concentration. In the so-called TML and CVCM tests a number of samples can be tested at one time. Each sample is placed into an aluminium foil boat. Following a 24-hour pre-conditioning at $25{ }^{\circ} \mathrm{C}, 50 \%$ relative humidity and standard atmospheric pressure to ensure that the samples receive common preliminary treatment, the individual samples are weighed. The samples are then loaded into individual compartments in a solid copper bar that can be heated. A cover, requiring that all volatile materials escape only through a small diameter exit port, closes each compartment. The sample is heated to $125^{\circ} \mathrm{C}$ by conduction and radiation for 24 hours. This causes the volatile materials to be driven off. A significant portion of the escaping volatiles collects on a disk if the condensation temperature is $25^{\circ} \mathrm{C}$ or above. The mass loss of the sample is determined from the weights before and after the $125^{\circ} \mathrm{C}$ exposure, and the percentage loss is calculated to provide the Total Mass Loss (TML). In a similar manner, the difference between the weight of a clean collector and of the collector having condensed materials will provide the mass of condensables. This mass of condensables is calculated as a percentage of the starting mass of the sample, and stated as CVCM. Materials having TML and CVCM equal to or lower than $1.0 \%$ and $0.1 \%$ respectively are considered as low outgassing.

The outcome of such testing is limited, as the resulting information does not identify pollutants. However, the existing list of low outgassing materials catalogued with this method is enormous and its outcome compatible with the more exhaustive tests carried out by irradiating counters. The accessible NASA database [8] contains more than 1600 entries for adhesives, 500 entries for rubbers and elastomers, 800 entries for potting compounds, etc. It can help pre-selecting assembly materials before doing more time-consuming tests.

\section{Outgassing rate of some assembly materials}

\subsection{Room temperature curing epoxy compounds}

Epoxy compounds that polymerize at room temperature are very attractive because they make it possible to assemble detectors having materials with very different expansion 
coefficients. Table 1 shows the list of epoxy compounds curing at room temperature and labelled as low outgassing.

\begin{tabular}{cccc}
\hline Epoxy & Outgas & Effect in Detector & Used by \\
\hline STYCAST 1266 (A+B) & NO & NO & CERN/GDD \\
STYCAST 1266 (A+Catalyst 9) & NO & NO & HERA-B/OTR \\
HEXCEL EPO 93L & NO & NO & Out of production \\
ECCOBOND 285 & NO & NO & HERA-B/TR \\
$\begin{array}{c}\text { ARALDITE AW103 } \\
\text { (Hardener HY 991) }\end{array}$ & NO & NO & $\begin{array}{c}\text { CERN/GDD } \\
\text { ATLAS/TRT }\end{array}$ \\
TRABOND 2115 & NO & NO & ATLAS/TRT \\
\hline
\end{tabular}

Table 1 Low outgassing epoxy compounds curing at room temperature. Both gas analysis and aging test have been carried out to characterize these glues.

As for subsequent tables, it is shown if any outgassing has been detected with the analysis station described in Section 3.1, and the effect of the outgassed pollutants, if any, in the monitored gain of a strongly irradiated gaseous detector. For materials considered as low outgassing the gas analysis should not reveal the presence of pollutants in the gas; the gas gain of the irradiated counter stays constant (measured with about $1 \%$ accuracy) up to accumulated doses above $0.5 \mathrm{C} / \mathrm{cm}$ of wire. The TML and CVCM values classify all the tested epoxies as low outgassing except Stycast 1266 and Araldite 103. However, these two-epoxy compounds have been thoroughly tested and validated irradiating counters. All components shown in the table are presently employed by different experiments. Stycast 1266 (parts A and B) has been widely used to assemble MSGCs. It has good moisture resistance, electrical properties and good adhesion to metal, glass and plastics. Yet its long curing time (>16 hours) forced the Outer Gaseous Tracker of the Hera-B experiment [9] to try a new hardener suggested by the producer: catalyst 9 mixed with Stycast part A. The new combination decreases the curing time by a factor 3 while keeping excellent outgassing properties. The inner tracker of Hera-B, a set of hundred detectors based on the MSGC and GEM technology [10] makes use of Eccobond 285 in the assembly steps where room temperature curing is mandatory. Araldite AW 103 mixed with HY 991 hardener is presently used as sealing glue for the straws of the Transition Radiation Tracker (TRT) of ATLAS [11] and for construction of the triple-GEM stations of the COMPASS experiment [12]. Tra-Bond 2115 is used in specific parts of the TRT assembly, where its excellent capillary effect helps the construction procedure. 


\begin{tabular}{cccc}
\hline Source & Epoxy & Outgas & Effect in Detector \\
& ARALDITE AW 106 & YES & YES \\
CERN/GDD & Hardener HV 935 U & & \\
ATLAS/TRT & DURALCO 4525 & YES & YES \\
CERN/GDD & DURALCO 4461 & YES & YES \\
CERN/GDD & HEXCEL A40 & YES & - \\
CERN/GDD & TECHNICOLL 8862 & YES & - \\
CERN/GDD & Hardener 8263 & & \\
& NORLAND NEA 155 & YES & - \\
CERN/GDD & EPOTEK E905 & YES & - \\
CERN/GDD & NORLAND NEA 123 & YES & - \\
CERN/GDD & & & \\
& &
\end{tabular}

Table 2 List of epoxy compounds curing at room temperature for which the GC detects some pollutants at the ppm level. For some epoxy compounds, an aging test of a validated gaseous detector has been carried out. Permanent gas gain variations have been detected.

Table 2 lists the low temperature curing epoxy compounds for which the gas analysis station detects some pollutants at the ppm level. For some of them an aging test of a validated detector has been additionally carried out [13]. In such cases, permanent gas gain variations have been detected. A sample case is Araldite AW106 (and hardener HV 935 U). This epoxy, cured at room or higher temperatures, has been commonly used for gaseous detector assembly due to the excellent mechanical, electrical and radiation resistance properties. Aging tests and several gas analyses have been performed to ascertain its possible effect on aging. During the aging test the gas gain of the irradiated counter, connected downstream the box containing the sample, decreases when the Araldite AW106 is heated up to speed up the rate of pollutants outgassing (Fig. 4). Removing the sample from the gas stream slightly recovers the gain but it never reaches the original value. The counter response remains degraded even after flowing clean gas for several days, indicating a permanent damage. Fig 5 shows the M/Z spectrum of the analyzed gas flowing out of the heated box containing the Araldite 106 [14]. The outgassing is present at low temperatures but increases significantly if the sample is heated. Pollutants such as butane, hexane, trimethyl pentane and trimethyl butane are detected and identified with the MS detector. The values for TML and CVCM are $3.26 \%$ and $0.02 \%$ respectively [15], classifying this epoxy as non-low outgassing according to NASA standards.

\subsection{High temperature curing epoxy compounds}

Some epoxies curing at temperatures above $50{ }^{\circ} \mathrm{C}$ have been tested and they are listed in Table 3. For some epoxies it is possible to find that detection of outgassed compounds does not imply a bad effect in the response of an irradiated counter. This, for instance, is the case of Epotecny E505. This epoxy has been extensively used in the construction of MSGCs, which 


\begin{tabular}{cccccc}
\hline Source & Epoxy & $\begin{array}{c}\text { Curing T } \\
\left({ }^{\circ} \mathbf{C}\right)\end{array}$ & Outgas & $\begin{array}{c}\text { Effect in } \\
\text { detector }\end{array}$ & Global result \\
\hline CERN/GDD & EPOTECNY E505 SIT & 50 & YES & NO & OK \\
HERA-B/TTR & EPOTEK H72 & 65 & YES* & NO & OK* \\
CERN/GDD & AMICON 125 & 85 & NO & - & OK \\
CERN/GDD & POLYIMIDE & 65 & NO & - & OK \\
& DUPONT 2545 & & & & OK \\
ATLAS/TRT & RUTAPOX L20 & 60 & NO & - & BAD \\
CERN/GDD & ARALDITE AW 106 & 70 & YES & & BAD \\
CERN/GDD & LOCTITE 330 & & YES & YES & BAD \\
CERN/GDD & EPOTECNY 503 & 65 & YES (Silicone) & & BAD \\
CERN/GDD & NORLAND UVS 91 & 50 & YES & - & \\
\hline
\end{tabular}

Table 3 List of investigated epoxy compounds curing at temperatures above $50^{\circ} \mathrm{C}$.

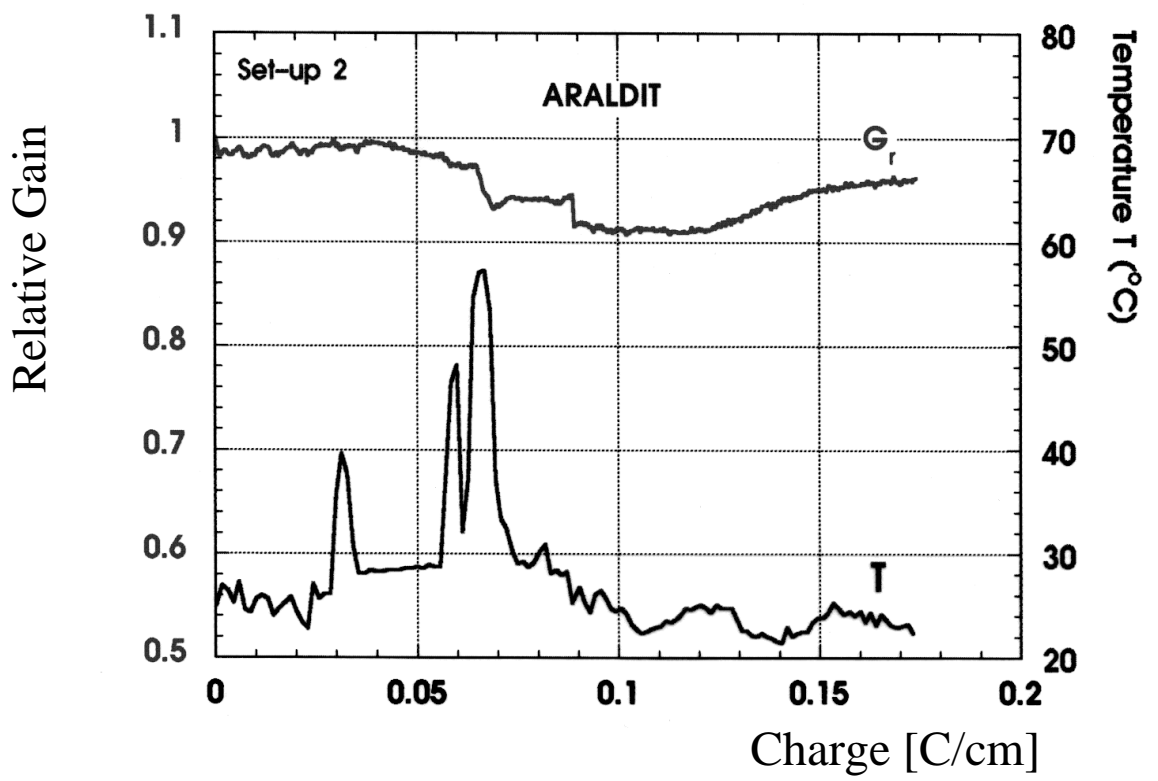

Figure 4 Normalized gas gain as a function of accumulated charge when the operating gas, Ar-DME (90-10), is in contact with Araldite 106 (and hardener HV $935 \mathrm{U}$ ) samples. The temperature of the sample along the test is also shown. 


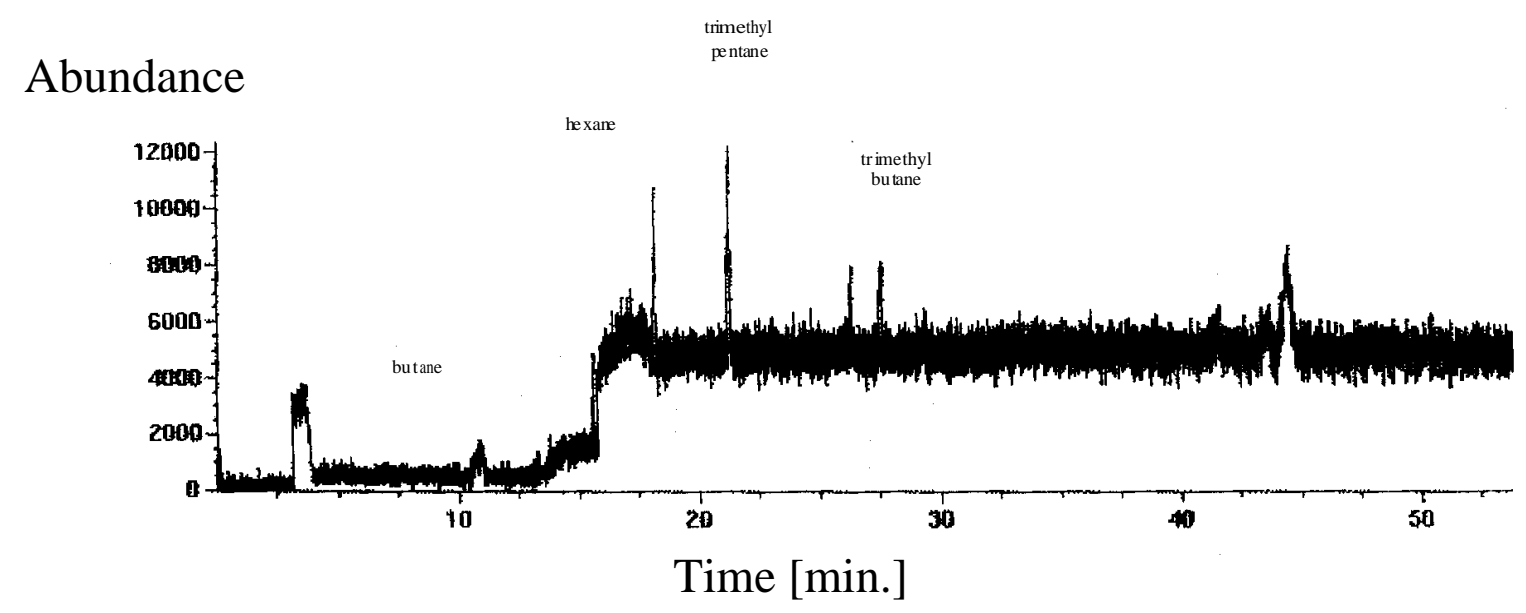

Figure $5 \mathrm{M} / \mathrm{Z}$ spectrum of the gas analyzed as it comes out of the box containing the Araldite 106 sample heated up to $60^{\circ} \mathrm{C}$. Several compounds are detected being their quantity some tenths of ppm.

have successfully followed numerous, successful, long-term aging tests. Epotek H72, used for the assembly of MSGC+GEM detectors presently running in Hera-B, slightly pollutes the operating gas in the firsts hours of contact with it. Though the irradiated counter shows very stable behaviour under irradiation, indicating that the detected pollutant might be volatile and it is easily removed from the system thanks to the gas flow.

\subsection{Other adhesives}

Table 4 shows the conductive epoxies that have been tested. All of them have not induced detectable gas pollution. During aging tests performed with the final detectors no aging effect could be correlated with the use of these conductive epoxies. Table 5 shows the outgassing rate of adhesive tapes.

Some sealing materials might contaminate the interior of an otherwise clean and contamination-free detector. Table 6 lists the results observed when testing some sealants for detectors and gas systems. Varian Torr seal is often used as leak sealer in ultra high vacuum applications due to the known low outgassing rate. Even if some outgassing is detected one should note that this components are usually employed in very small quantities to fix small gas leaks. This would explain why for instance the pollution outgassed from DOW CORNING R4-3117 RTV does not affect the response of the irradiated detector where few leaks have been potted with few milligrams of this sealant [16]. Besides, as we shall see in section 4.6, silicone-containing compounds should be generally avoided for the construction of high rate gaseous detectors. 


\begin{tabular}{|c|c|c|c|c|}
\hline Source & Epoxy & Outgas & Effect in Detector & $\begin{array}{l}\text { Global } \\
\text { Result }\end{array}$ \\
\hline CERN/GDD & TRADUCT 2922 & NO & & $\mathrm{OK}$ \\
\hline HERA-B/OTR & $\begin{array}{l}\text { SILBER LEITKLEBER } 3025 \\
(\mathrm{~A}+\mathrm{B})\end{array}$ & NO & NO & $\mathrm{OK}$ \\
\hline ATLAS/TRT & TRABOND 2902 & NO & NO & $\mathrm{OK}$ \\
\hline
\end{tabular}

Table 4. Outgassing tests of conductive epoxies.

\begin{tabular}{ccc}
\hline Source & Name & Outgas \\
\hline HERA-B/OTR & SCOTCH 467 MP & YES \\
HERA-B/OTR & TESAFIX 4388 & YES \\
\hline
\end{tabular}

Table 5 Gas analyses of adhesive tapes.

\begin{tabular}{|c|c|c|c|c|c|}
\hline Source & Material & Type & Outgas & Effect in Detector & $\begin{array}{l}\text { Global } \\
\text { Result }\end{array}$ \\
\hline CERN/GDD & VARIAN Torr-Seal & Solvent-free epoxy resin & $\mathrm{NO}$ & $\mathrm{NO}$ & OK \\
\hline CERN/GDD & RHODORSIL CAF4 & Caoutchouc Silicone RTV & NO & $\begin{array}{c}\mathrm{NO} \\
\text { in very small quantities }\end{array}$ & OK? \\
\hline CERN/GDD & $\begin{array}{l}\text { DOW CORNING } \\
\text { R4-3117 RTV }\end{array}$ & Silicone based & YES & $\begin{array}{c}\mathrm{NO} \\
\text { in very small quantities }\end{array}$ & OK ? \\
\hline HERA-B/OTR & LOCTITE 5220 & Polyurethane-based & YES & - & BAD \\
\hline
\end{tabular}

Table 6 Outgassing tests carried out for some sealants used for fixing small gas leaks in chambers and gas systems. 


\begin{tabular}{cccccc}
\hline Source & Material & Type & Outgas & $\begin{array}{c}\text { Effect in } \\
\text { Detector }\end{array}$ & $\begin{array}{c}\text { Global } \\
\text { Result }\end{array}$ \\
\hline CERN/GDD & STESALIT 4411W & Fibreglass & YES & NO & OK \\
CERN/GDD & VECTRA 150 & Liquid Crystal Polymer & YES & NO & OK \\
CERN/GDD & PEEK Crystalline & Polyeteherether ketone & NO & NO & OK \\
ATLAS/TRT & ULTEM & Polyetherimide & NO & OK \\
ATLAS/TRT & C-Fiber & C-fibre & NO & - & OK \\
ATLAS/TRT & POLYCARBONATE & C-fibre & NO & - & OK \\
HERA-B/ITR & FIBROLUX G10 & Fibreglass & YES & - & BAD \\
HERA-B/ITR & HGW 2372 EP-GF & Fibreglass & YES & YES & BAD \\
CERN/GDD & RYTON & Polysulphur phenylene & YES & YES & BAD \\
CERN/GDD & PEEK Amorphous & Polyetherether ketone & YES & -
\end{tabular}

Table 7 Rigid materials tested for outgassing.

\subsection{Rigid assembly materials}

The selection of rigid materials for use in detector assembly is a very important part of the design. Not only must the material be capable of being fabricated into required components but it must stand up to the environmental conditions. For high rate detectors, low pollutant outgassing should be considered as one of the deciding factors. Table 7 lists the rigid materials that have been tested. Gas pollution to the ppm level is detected for standard fibreglass materials. A replacement candidate is Stesalit $4411 \mathrm{~W}$ (epoxy without filler glass fibre mat). It has good machining, excellent mechanical and electrical properties and proved low outgassing properties [13]. Rigid materials such as ULTEM, Polycarbonate, VECTRA and Ryton have the advantage that can be moulded into assembly pieces. Ryton has shown large rate of gas pollution accompanied by a dramatic effect on the gas gain of an irradiated counter [13]. VECTRA is a low outgassing plastic of smetic (or liquid crystal) structure with interesting properties [14]; for example, two Vectra pieces can be welded with a heating plate providing after polymerisation homogenous leek proof connections. PEEK is a new high performance thermoplastic with excellent radiation resistance, resilient to most chemicals and relatively inexpensive. Amorphous PEEK (type Victrex) shows some degree of outgassing as well as mechanical changes and discoloration if tested with DME. However, if the material is thermally annealed to produce crystalline PEEK, this plastic seems to be an excellent choice. 


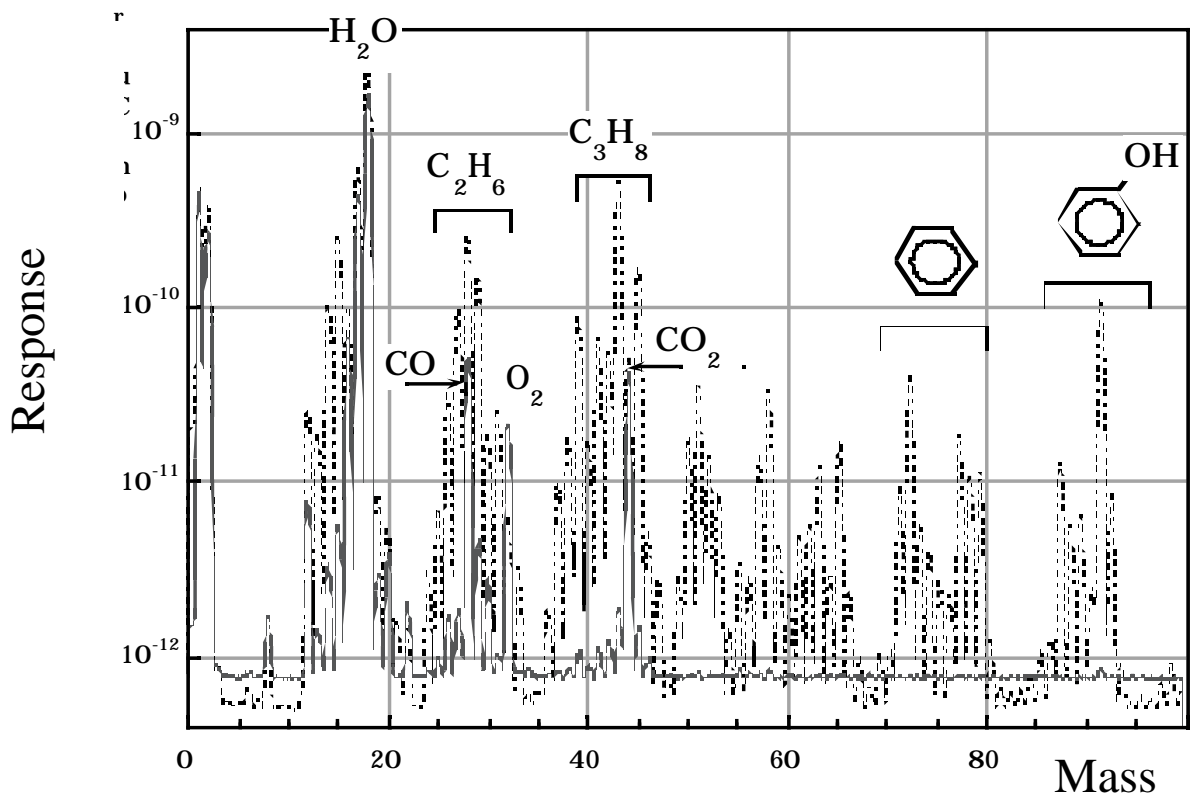

Figure 6 Response of an ion analyzer for two samples of Nuvovern LW, a two-component Polyurethane [15]. The sample showing heavy outgassing has been processed using the component hardener few days after the recommended expiration date.

One should take into account that high-temperature and pressure-moulded plastics are not free of surface contaminating materials. If they are not moulded with exactly the right process conditions some outgassing can be expected. This can occur even if the mechanical properties meet specification requirements. Another source is the mould release agent. Many of them are silicone-based.

\subsection{Accidental contamination}

A source of contamination usually underestimated turns up from the incorrect processing of materials by incorrect weighing, mixing or use of an insufficient or incorrect cure cycle. Fig. 6 [15] shows the response of an ion analyzer for two samples of Nuvovern LW, a twocomponent Polyurethane. Both samples have been processed under identical conditions; for one of them the hardener was used a few days after the recommended expiration date. The spectrum shows for both samples outgassed volatile components such as oxygen, water, $\mathrm{CO}$ and $\mathrm{CO}_{2}$, probably adsorbed by the polyurethane during the mixing process. The sample improperly processed shows in addition some hydrocarbons of high molecular weight and heavy aromatic components typical of components that have not properly cured.

An adhesive will outgas more while it is being cured than after it is cured. Glue curing under clean gas flow will help removing reaction products released during room temperature.

\subsection{Silicon contamination}

Silicone has been systematically found coating aged chambers, although in many cases the origin of the pollutant has not been clearly identified. Probably the pollutant was part of the 
assembly materials or components in the gas system. Possible sources are silicone rubber sealants, potting and encapsulation compounds, silicone adhesives, silicone vacuum grease used to lubricate O-rings, mould-release agents, polluted gas cylinders, detergent residues (sodium metasilicate), glass and related products such as glass fibres used for reinforcing resins, silicone oil commonly used in bubblers and diffusion pumps. Fig. 7 shows the unambiguous effect of a silicone bubbler placed downstream an irradiated MSGC, as compared to the response of the same detector connected to a long, clean exhaust line.

Silicone can polymerize both with hydrocarbons and oxygen to form rather heavy polymers, thus not easily removable by the gas flow. It should be taken into account that silicone has a high natural affinity for most materials and the tendency to migrate. It is relatively inert chemically and unaffected by most solvents, therefore among the most difficult surface contaminants to remove. It has recently been found that silicone can be etched away by Fspecies created in the gas avalanche [17] and spread out in the system, thus triggering polymerization processes.

\section{Detector assembly procedures}

Contaminates during assembly can make the best-designed detector to fail. As a general rule, the assembly area must be isolated from other manufacturing areas, and usually

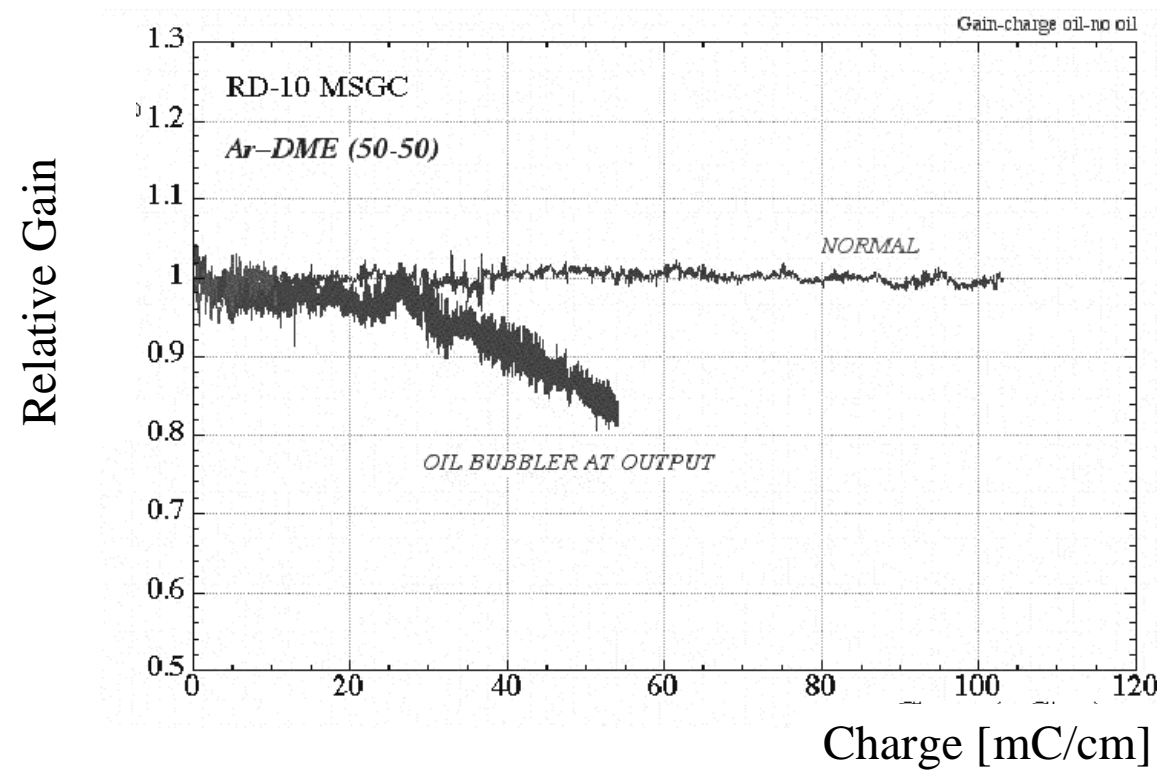

Figure 7 Lifetime of a MSGC irradiated in a clean gas system in Ar-DME (90-10) and the unambiguous effect of a silicon bubbler placed downstream the counter.

following clean room standards. Some equipment should be avoided or protected. Examples of contamination are tools with lubricated shafts, soldering or brazing equipment that requires 
heating of volatile fluxes, motors and vacuum pipes with outgassing oils. Another important source of contamination is the personnel. The people in the production should know the reason why something must be done in a certain manner; hence careful and exhaustive training is needed. Examples of contamination are street clothing; hair, make-up, fingernail and fingerprints are source of oil and particulates and many creams and cosmetics contain silicones. Proper education will greatly decrease problems in the manufacturing.

The assembly process has to be well specified and stable. It should be rechecked periodically and only include proved improvements result of a careful observation of the process. A good assembly process includes procedures about cleanliness of assembly components and its verification. Tracing capability is also an important feature, especially when building very large systems.

\section{Gas system components}

Components used to build gas systems have to be selected with care to avoid unexpected pollution of an initially clean gas mixture. The components choice should include the selection of materials which are low outgassing and chemically compatible with the gas mixture. These principles should be used to assemble, not only the final gas mixer, but also any system supplying gas during the assembly process and testing of detectors in the laboratory.

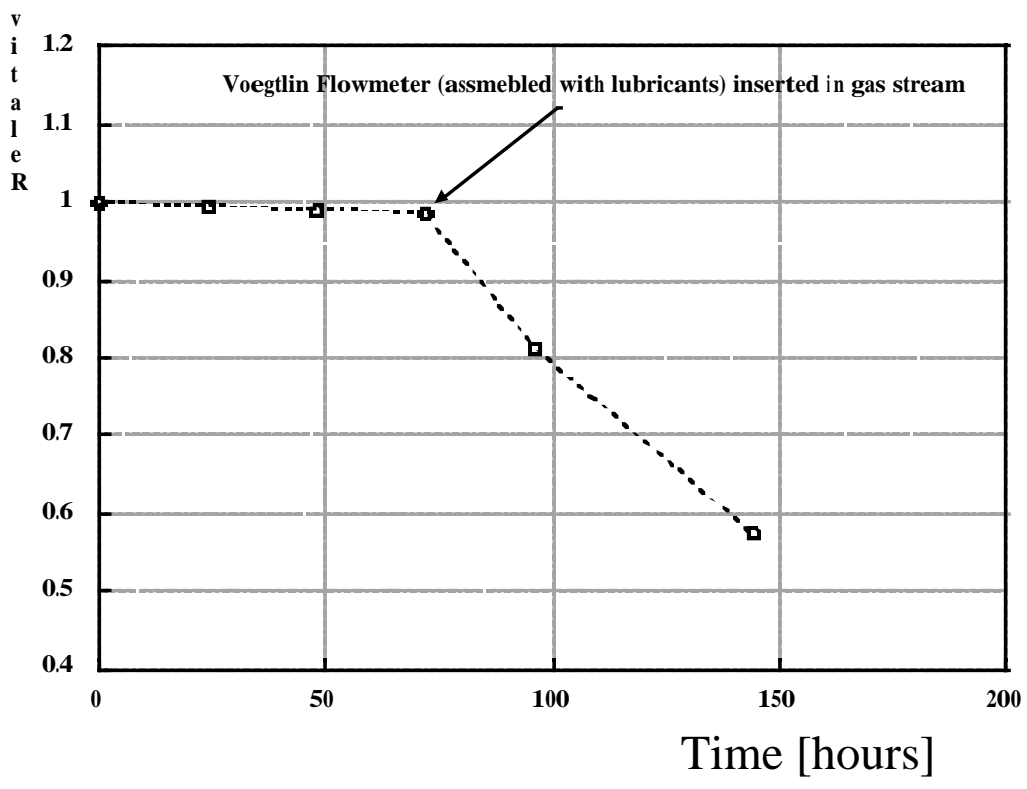

Figure 8 Effect of pollutants released by a standard flowmeter assembled with lubricated joints and needle valve on the gain of a ATLAS/TRT straw tube.

Fig. 8 shows the response of an irradiated ATLAS/TRT straw tube operated in a clean gas system supplying $\mathrm{Ar}-\mathrm{CF}_{4}-\mathrm{CO}_{2}$ [63-10-27]. When a standard flowmeter, constructed with a lubricated valve and few greasy Viton O-rings, is inserted in the gas stream after about 70 hours of continuous irradiation, the gain of the straw falls very fast due to immediate polymerization processes triggered by released pollutants. A procedure to clean the flowmeter 
[18] consists of disassembling and cleaning all parts in a series of ultrasonic baths ${ }^{4}$ of isopropyl alcohol, followed by a clean distilled water bath. A new alcohol volume is used for each bath. The parts are then heated to $70{ }^{\circ} \mathrm{C}$ before the flowmeter is remounted. Fig. 9 shows the normalized gas gain of three straws irradiated under the same conditions as shown in Fig. 8 , but with the cleaned flowmeter in the gas system. This practice should be used if any doubt would exist about the cleanliness of other parts of the system, such as pipe connectors. Other complex components, such as valves and cylinder pressure regulators are also potential polluting sources. Components used for ultra high purity applications are usually assembled following strict cleanliness requirements. However, a careful selection of joints still has to be made in terms of gas compatibility and outgassing. Table 8 shows the results of some tests carried out for some commonly used O-rings. Viton, one of the most common materials, has shown some outgassing at the ppm level, though it has been satisfactorily used in very clean gas systems.

\begin{tabular}{ccccc}
\hline Material & Type & Outgas & $\begin{array}{l}\text { Effect in } \\
\text { Detector }\end{array}$ & Global Result \\
\hline KALREZ & Fluoropolymer & NO & NO & OK \\
VITON & Fluorinated copolymer & YES & YES & BAD \\
EPDM & Copolymer ethylene propylene & YES & - & BAD \\
& & & & BAD \\
PVDF & Fluorinated polyvinyldene & YES & & \\
\hline
\end{tabular}

Table 8 Results of tests carried out for some commonly used O-rings.

\footnotetext{
4 The ultrasonic bath should be performed following some basic rules: the components to clean are placed in a small volume of solvent in a beaker made out of glass or stainless steel, with a flat bottom. The beaker is suspended in water, that acts as a sonic coupling medium. Fluid levels in the tank and container should be more or less matched, and the volume of parts to be cleaned should be limited to less than about $25 \%$ of the fluid volume. For complex parts, several changes of orientation may be required for careful cleaning. Cleaned items should be removed from solutions while the unit is still operating, to provide a thorough rinsing action.
} 


\begin{tabular}{|c|c|c|c|c|}
\hline Material & Type & Outgas & $\begin{array}{l}\text { Effect in } \\
\text { Detector }\end{array}$ & Global Result \\
\hline $\mathbf{P P}$ & Polypropylene & NO & NO & OK \\
\hline $\begin{array}{l}\text { RILSAN } \\
\text { NYLON }\end{array}$ & Polyamide & Water & NO & $\mathrm{OK}^{*}$ \\
\hline PEEK Crystalline & Polyetherether ketone & NO & NO & OK \\
\hline $\begin{array}{c}\text { PEEK } \\
\text { Amorphous }\end{array}$ & Polyetherether ketone & YES & - & BAD \\
\hline PEE & & YES & - & BAD \\
\hline PUR & Polyurethane & YES & - & BAD \\
\hline
\end{tabular}

Table 9 Outgassing properties of some plastic piping.

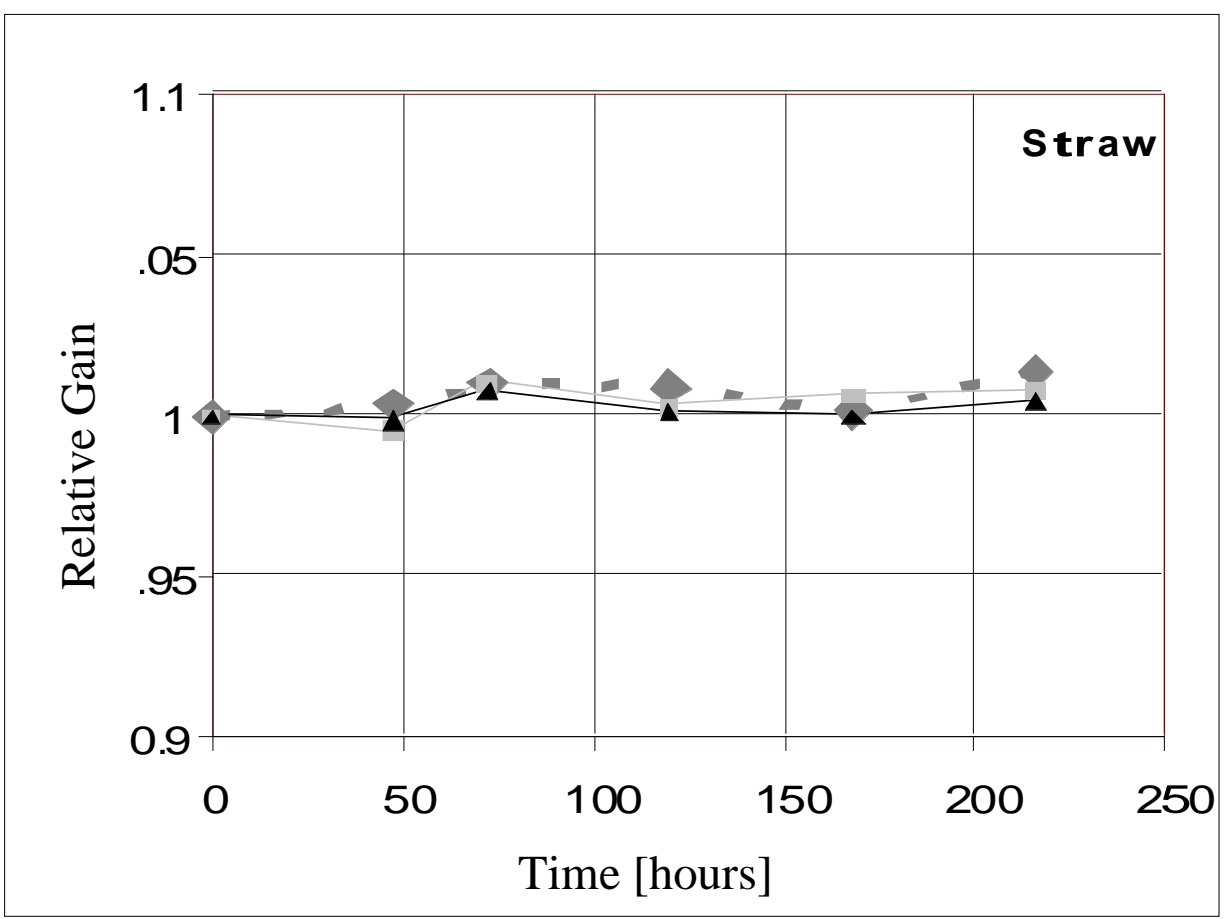

Figure 9 Response of three ATLAS/TRT straw tubes irradiated in a validated clean gas system, which is modified to insert a Voegtlin flowmeter after it has been disassembled and thoroughly cleaned in a series of ultrasonic baths to remove lubricants.

Plastic piping is often used because of its insulating properties, elasticity, low density and price as compared with the reliable, electropolished, stainless steel pipes. In order to obtain the desired physical characteristics, these complex materials are frequently modified chemically 
or with additives. As a result, they are often made more susceptible to outgassing. Table 9 shows the list of some plastic pipes that have been tested. Generally plastics should be kept to a minimum due to their high gas permeability. At test laboratories, nylon piping can be used, for instance, as a clean and simple way of adding a small concentration of water to the gas mixture, due to the natural outgassing of water from or diffusion through the tube walls.

A major problem when selecting clean gas components is the difficulty to find complete specifications and product descriptions; usually they do not contain enough information. Even more, once an adequate product has been found and successfully tested following a strict validation process that includes long-term aging tests of counters, it is difficult to trust the cleanliness conditions for larger quantities. The golden rule to obtain a reliable gas system seems to be the definition of the cleanliness requirements by understanding precisely the effect of particular pollutants in the detector, to avoid pollution during the assembly process, and to flush gas through the open system as much and as soon as possible to clean up possible pollutants.

\section{Some factors affecting aging rate in some gases}

The operational parameters of gaseous detectors such as gas gain, discharge probability, signal characteristics and others have been optimized for different gas mixtures. Nowadays there is still some controversy about the choice of the optimal gas mixture, first because the selection depends on the particular experiment, and second, for high rate experiments the aging of the detector has to be kept to a minimum. Few gases have proved to be stable under such harsh conditions. We shall see the factors that might degrade the detector response for few of the commonly used gases.

\subsection{Hydrocarbon mixtures}

The use of hydrocarbons as quenching gas in proportional counters has been a rather popular choice because it gives an excellent energy resolution and small variations in gain. These advantages counteract its known tendency to polymerize $[19,20]$ and the fact that it has been often found that this gas does not provide very long lifetimes. However, as aging of gaseous detectors depends on a large number of parameters, several tests with $\mathrm{Ar}_{-} \mathrm{CH}_{4}$ 90-10 have been done varying material of the electrodes of the counter, gas purity and radiation 


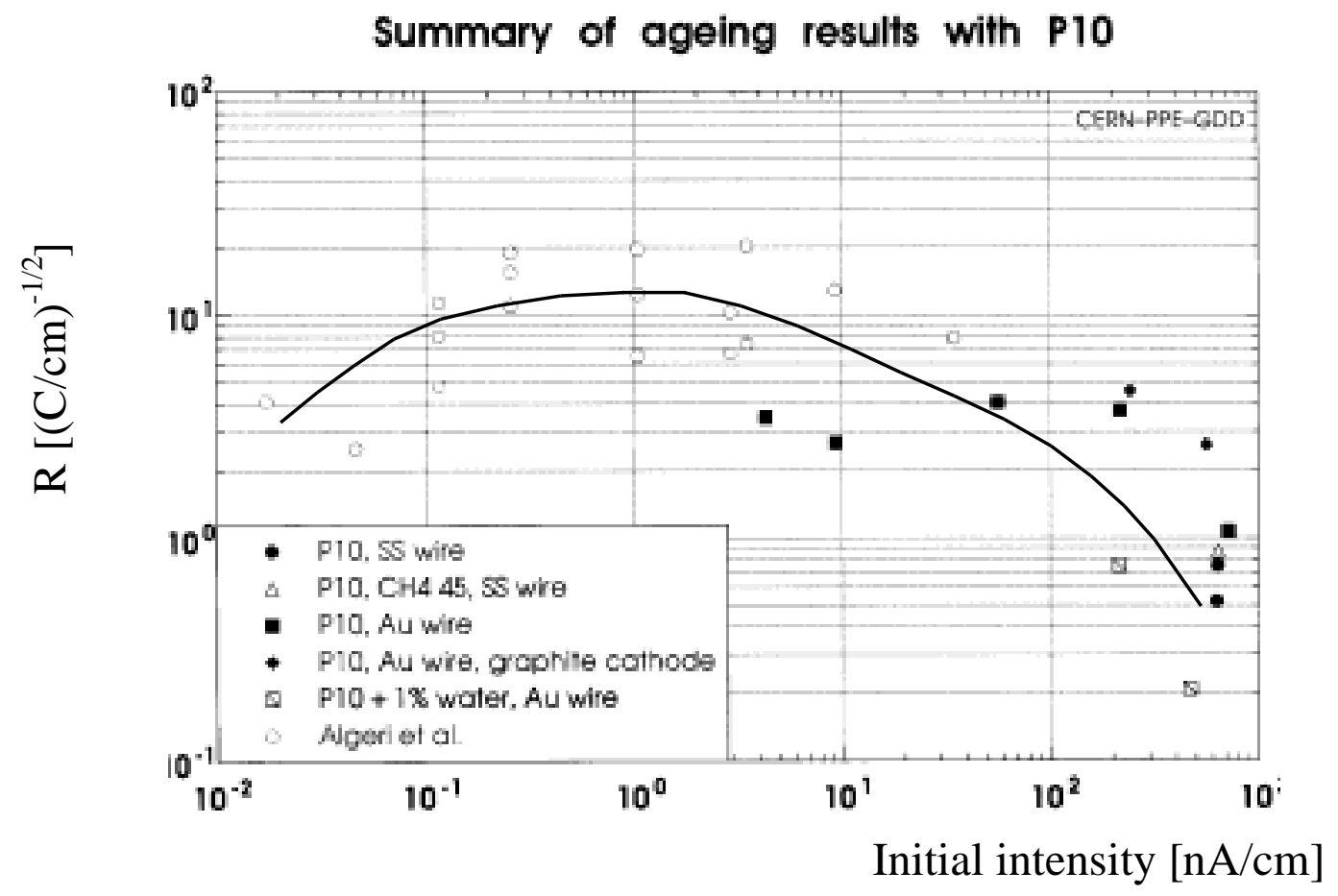

Figure 10 Aging rate characterized by the $\mathrm{R}^{\prime}$ value in $(\mathrm{C} / \mathrm{cm})^{-1 / 2}$ against the initial current in $\mathrm{nA} / \mathrm{cm}$ of wire drawn. All counters have operated in P10 varying some conditions.

dose, in order to discern which parameters play an important role in the aging process [21]. This study serves as a sample case as probably the observations apply to any hydrocarbonbased mixture. Fig. 10 shows, for the totality of tests with this mixture, the level of aging

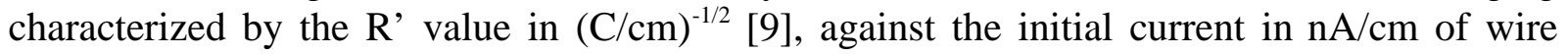
drawn in the counter, mainly defined by the product of gain by particle rate. $\mathrm{R}^{\prime}$ is defined as

$$
G_{r}=a e^{\left(-R^{\prime} \sqrt{Q}\right)}
$$

being $G_{r}$ the gas gain relative to the initial one and $Q$ the accumulated charge expressed in $\mathrm{C} / \mathrm{cm}$. The R' value does not depend on the particular dose accumulated in the aging run.

Several conclusions might be extracted from Fig. 10:

- Independently of the materials of the electrodes and, more importantly, of the purity of methane, the rate of aging for P10 does not vary considerably for a given set of initial conditions. This would suggest that the methane itself polymerizes in the avalanche plasma and produces deposit layers on the wire surface.

- The rate of aging normalized per unit of charge and length is not constant, but depends on the charge collection rate during the aging test. In plasma chemistry it is known that radiation-induced polymerization is a function of the square root of the dose [1]. At large irradiation rates, the density of ions and radicals will be lower due to spacecharge effects. This weakens the field and the avalanche extends over larger regions; 
the rate of polymerization would decrease, and in turn, so would the concentration of deposits on the wire. This tendency permits to suppose that in real experiments, where the irradiation conditions are not as drastic as in the laboratory tests, a faster aging rate can be expected.

- The rate of aging improves if water (at \% level) is added. This is in accordance with the generally accepted idea that oxygen containing molecules, among others, prevent to some extent the polymer chains growing. It might also be that water gives some electrical conductivity to thin insulating films deposited on the wires. However, for these tests, the improvement is still limited to consider this mixture as an operative gas in high luminosity experiments.

\subsection{Dimethyl ether}

Dimethyl ether appeared in the 1986 Aging Workshop as a reliable quencher for gaseous detectors operated at high rates. Since then, many groups have studied the aging behaviour of DME-based mixtures. Fig. 11 [21] compares the lifetime of identical irradiated counters under $\mathrm{Ar}_{-} \mathrm{CH}_{4}$ and Ar-DME in proportions 90-10. The counter in DME accumulates a large dose without major degradation of its properties. However some gain reduction is still observed in aging tests with DME. This may be probably due to a progressively larger amount of pollutants in the gas mixture as the DME cylinder is being used. Fig. 12 shows the aging rate, expressed by the R' parameter, as function of DME used in the cylinder during the tests. Certain impurities present in the liquid DME enter in the gaseous phase through a selective process called Rayleigh distillation. For the tests shown in the figure, the pollutants detected by gas chromatography when the DME cylinder has been used around $25 \%$ are freon 11 and 12 and few hydrocarbons. Their concentration stays anyhow at the ppm level. The triangle point corresponds to the addition of water to the gas mixture that seems to prevent the aging process. The solution is simple but might not be applicable to all kind of detectors; water concentration should probably be adjusted for the running conditions of each particular detector.

DME has proved to work for high rate detectors. Yet it has a very high sensitivity to traces of pollutants at ppb level, which are difficult to control. In addition, being a rather reactive gas, time-consuming studies have to be performed to check the resistance of DME to some common materials used in chambers. For instance, and as it is shown in Table 10, the immersion for 2 hours of conventional detector assembly materials in liquid DME shows some degradation, measured in this case as percentage of swelling. Even if laborious laboratory work is done to find a set of adequate assembly materials, it remains to be verified if these clean conditions can be maintained in complex systems. DME has been widely replaced by $\mathrm{CO}_{2}$, which seems to be less sensitive to small pollution and non-flammable, the cost being the increase of operating voltage and the larger energy of discharges, with all the consequences on chamber operation. 


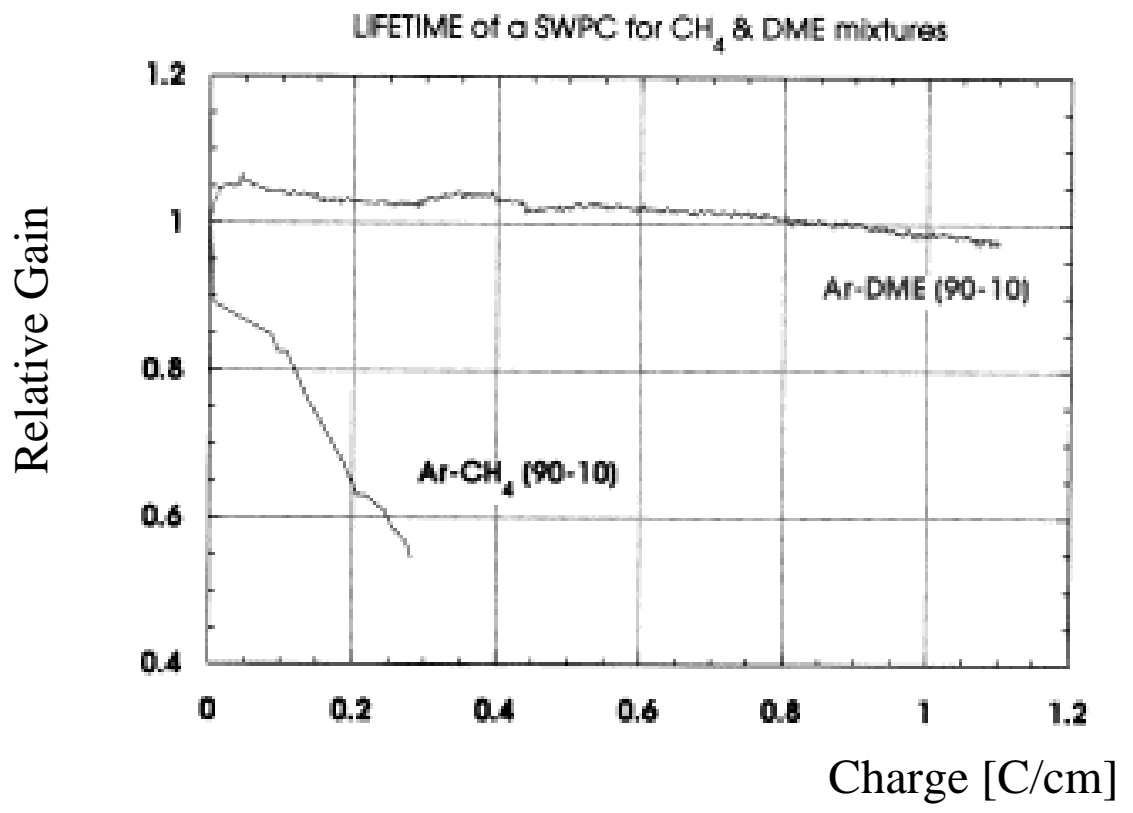

Figure 11 Comparison of the response of a clean single wire proportional counter irradiated in $\mathrm{Ar}-\mathrm{DME}$ and $\mathrm{Ar}-\mathrm{CH}_{4}$.

\begin{tabular}{cc}
\hline Material & Swell (\%) \\
\hline LDPE (foil) & -0.6 \\
VECTRA & 0.0 \\
PEEK Crystalline (pipe) & +0.3 \\
KALREZ (joint) & +1.6 \\
PE & +1.8 \\
Kel-F (joint) & +4.0 \\
PCTFE (joint) & +4.0 \\
PVDF (joint) & +5.0 \\
Kapton (foil) & +5.4 \\
PEEK (Amorphous (pipe) & +8.7 \\
\hline
\end{tabular}

Table 10. Effect of the exposure of some conventional materials used in detectors or gas systems to 2 hours of liquid DME. 


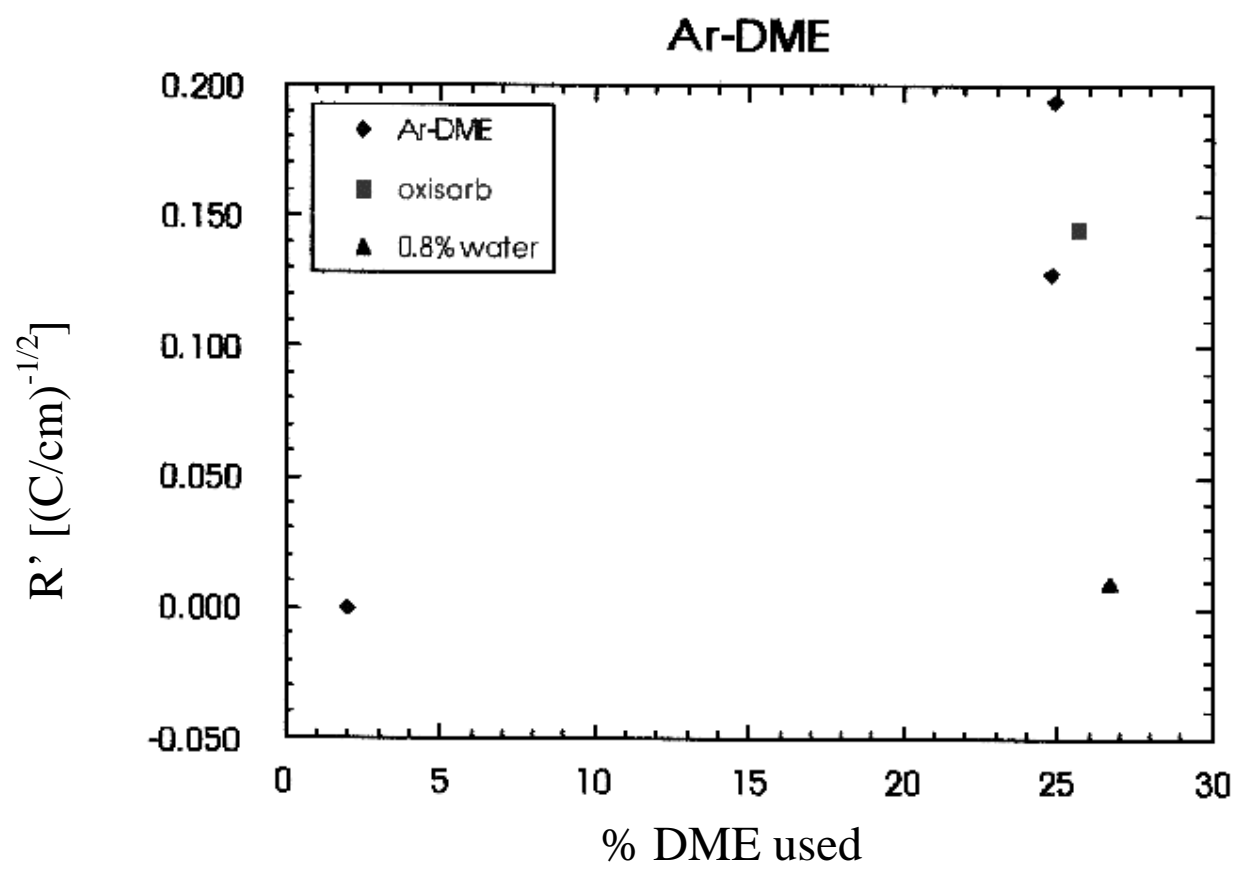

Figure 12 Aging rate for several tests carried put with Ar-DME (90-10) expressed as R' as function of the use of the DME cylinder. The triangle point corresponds to the rate of aging measured when $0.8 \%$ of water is added to the gas mixture.

\section{3. $\mathrm{CF}_{4}$}

The use of $\mathrm{CF}_{4}$, known as an aging preventing agent even for small concentrations in the mixture, has progressively been adopted for most gaseous detectors operated at high rates and needing high electron drift velocities. A balance between polymerization and etching processes is established depending on the charge density (particle rate and gas gain), the relative concentration of gas components and maybe the gas flow. The right balance permits achieving lifetimes above tenths of $\mathrm{C} / \mathrm{cm}$ for the straw tubes of the ATLAS experiment [17]. It appears, however, that in gaseous detectors etching is the favoured process: CF4 dissociates in rather stable species, $\mathrm{F}$ and $\mathrm{CF}_{\mathrm{X}}$ radicals. They have enough energy to break chemical bonds in polymers which are then reduced to stable, volatile products. These products are probably removed from electrodes by the gas flow. Few years ago this belief led to thinking that using $\mathrm{CF}_{4}$-based mixtures would allow the requirements for cleanliness in experiments to be relaxed. Presently, it has been shown that these mixtures are still sensitive to pollutants, as the balance between deposition-polymerization greatly depends on the species added to the $\mathrm{CF}_{4}$. Detailed studies have been performed to find wires resisting etching in $\mathrm{CF}_{4}$ mixtures. It has been found [22] that non-golden wires react with fluorine radicals produced in the avalanche to form metal fluorides, which may promote further deposition. Gold wires are essentially inert and have therefore good aging properties. However, strong etching effects have been observed in presence of water quantities above $0.1 \%$. The magnitude of the etching destruction depends, too, on the wire production technology and gold thickness [17]. 


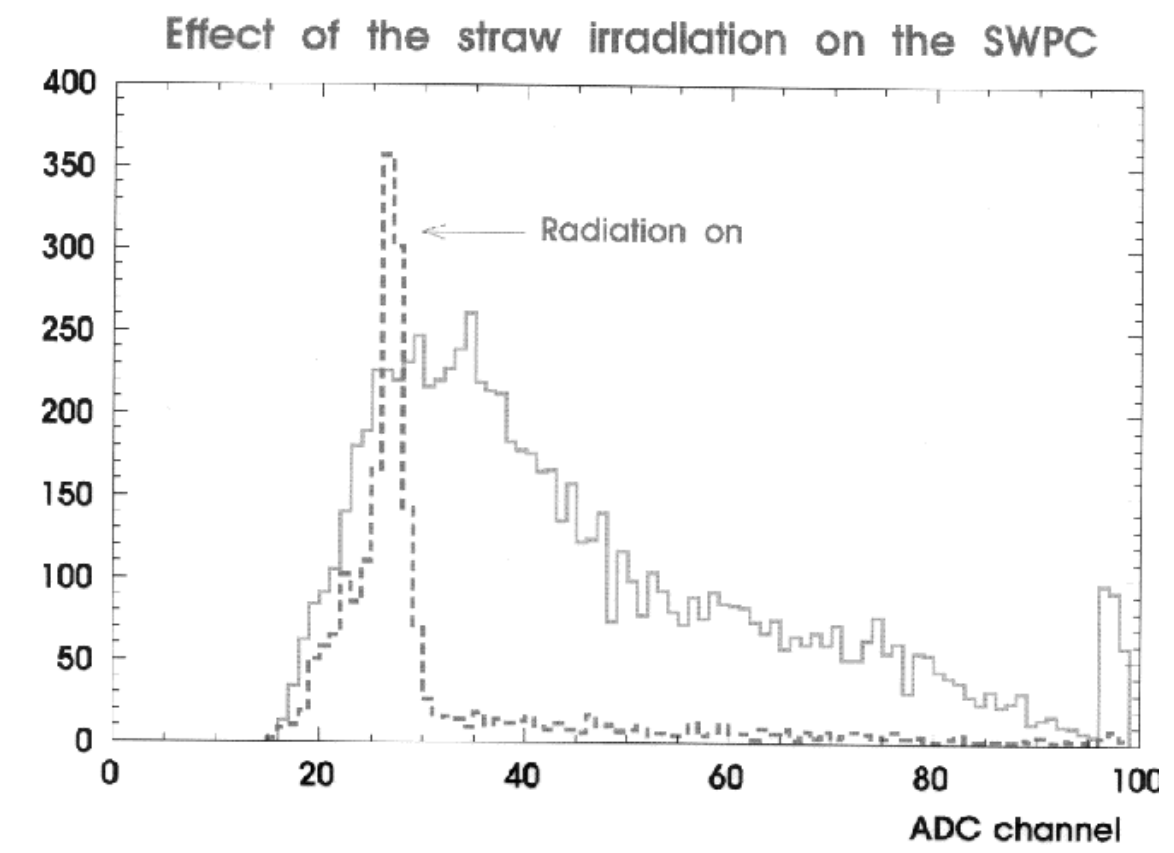

Figure 13 Pulse height measured in a single wire proportional counter ( $3 \mathrm{~cm}$ drift distance), which is gently irradiated but connected downstream a small diameter straw tube in $\mathrm{Xe}-\mathrm{CF}_{4}-\mathrm{CO}_{2}$ (50-30-20). When the straw tube is strongly irradiated the large energy pulses (continuous line) are suppressed due to the effect of electronegative species created in the avalanche.

Fig. 13 shows the pulse height measured in a single wire proportional counter, which is gently irradiated but connected downstream a $4 \mathrm{~mm}$ diameter straw tube flushed with $\mathrm{Xe}-\mathrm{CF}_{4}$ $\mathrm{CO}_{2}$ (50-30-20). When the straw tube is strongly irradiated, the wire counter pulse height is dramatically modified. Pulses with larger amplitude are suppressed suggesting that the gas composition is modified by some relatively stable species created in the avalanche. Some neutral but electronegative radicals reach the counter, with a drift distance between electrodes (around 30 times bigger than in the straw) large enough to capture electrons. This dramatic effect would put under question if this gas, despite the excellent results in terms of aging, might be used for detectors operating over the long-term. Many non-metallic components of the gas system and assembly materials could be affected.

It has been also pointed out [23] that F-compounds are able to react with silicone compounds. It was suggested that $\mathrm{CF}_{4}$ could be use to clean up traces of silicone in large systems. Presently, it is observed that this effect might be a real danger for large systems, because the silicone compounds are effectively removed but distributed ubiquitously polluting the detector and promoting heavy polymerization.

\section{Conclusions and summary}

The present knowledge about aging of gaseous detectors does not allow us formulating a set of definite guidelines to avoid degradation of detectors under large radiation loads. Experience from experiments or laboratory, where conditions are better controlled, allow nevertheless to set some general principles that might help if not to prevent, to minimize aging effects. Such a 
multi-parameter process can only be understood after an efficient R\&D phase has defined a set of working conditions, which are particular to each type of detector and its conditions of use. Among the parameters to identify, the following should be clearly understood: selection of the gas mixture, gas gain and gas purity, which implies a careful selection of gas system components, assembly materials and procedures. The long-term capability can then be evaluated and extrapolated to real conditions if final prototypes are tested under conditions as close as possible to the final ones, and strictly controlled. Among these parameters, it seems mandatory to irradiate large areas with a charge density as close as possible to the one expected in the final experiment. Space charge effects and discharges should be avoided as they hide or increase respectively polymerization processes.

Summarized in tables we present the outgassing results for several materials that can be used to assemble gaseous detectors and gas systems. A list of low outgassing assembly materials exists, that includes epoxy compounds, rigid materials, sealants, elastomers, etc. The effect of materials that outgas at the ppm level has to be tested for each particular case. That is the case for materials such us Viton, Teflon, Polyurethane, etc. In general, there is no good or bad material. A material is adequate or not for a very particular type of detector and conditions of use. Existing data, obtained either from systematic outgassing studies or experience gained with detectors, has only a pre-selective character that can help designing a detector before carrying out more time-consuming tests. Once the material selection appears to be adequate, the ultimate validation consists of irradiating the detector assembled with the chosen materials. It seems, on the other hand, rather universal that silicone compounds that easily migrate (lubricant-type) should be meticulously avoided, especially with some gas mixtures. One should note that the sources of silicone contamination are enormous and hard to find.

Mixtures of noble gas and hydrocarbon are not trustable for long-term high rate experiments. Hydrocarbons tend to polymerize by themselves. It is only a question of time when polymers will deposit on the electrodes with enough thickness to disturb detection efficiency. It is accepted that oxygen-containing additives can improve detector lifetime in such mixtures. DME $\left(\mathrm{CH}_{3}-\mathrm{O}-\mathrm{CH}_{3}\right)$ has proven to give long lifetimes. However, a careful material selection for detector assembly and gas system components is required due to the high reactivity of this gas. Halogen contamination at $\mathrm{ppb}$ level shortens detector lifetime under DME. Such low pollution levels are difficult to monitor and to keep under control. $\mathrm{CO}_{2}$ seems to be the conventional replacement as an easy-to-use mixture. $\mathrm{CF}_{4}$-containing mixtures are very attractive in terms of aging due to the fine polymerization-deposition balance phenomena. It is expected that radical and ions issued from the avalanche process are chemically reactive, competing with any polymerization process promoted by pollutants in the gas. The competition between these two processes might slow down conventional aging effects. Unfortunately contradictory effects have been observed with $\mathrm{CF}_{4}$ mixtures. Being a promising radiation hard mixture, detailed studies are necessary to set the conditions under which a particular detector might satisfactorily work. They need strict control of pollutants (specially water and may be oxygen) to avoid shifting the fine etching-deposition balance. Fradicals are rather active. They clean up aged wires, but also are they able to attack some materials used in the assembly. They are capable, for instance, of etching away Si-compounds that may promote strong polymerization processes. Supporting this idea, semiconductor industry uses $\mathrm{CF}_{4}$ to etch silicone and silicon-oxide substrates. Chemical processes involved in 
this type of gas mixtures are very complex, and probably highly sensitive to minor impurities. This means that all possible precautions should be taken when designing detectors and associated gas systems.

\section{Acknowledgements}

I would like to express my thanks to F. Sauli. Most of the outgassing tests and systematic work on MSGCs presented in this review have been carried out, together with C. Garabatos, in the framework of the CERN RD-10 and RD-28 projects. For the most recent tests carried out at P. Chiggato's group (CERN-EST/SM/DA), I show gratitude to M. van Stenis for her availability and expertise preparing samples.

I would like also to thank D. Froidevaux and F. Dittus for their support and interest when realizing this work. A. Romaniouk has contributed with his expertise on straw tube detectors.

\section{References}

[1] Procedings of the Workshop on Radiation Damage to Wire Chambers, LBL-21170 (1986).

[2] J.A. Kadyk, Nucl. Instr. and Meth. A 300 (1991) 436.

[3] F. Sauli et al., RD-10 Proposal, CERN DRDC 92-40, DRDC/P9 Rev., (1990).

[4] R. Bouclier et al., Nucl. Instr. and Meth. A 381 (1996) 289.

[5] A. Romaniouk, ATL-INDET-98-211 (1998).

[6] R. Bouclier et al., Nucl. Instr. and Meth. A 348 (1994) 109.

[7] M. Capeans, Ph.D. Thesis, Univ. Santiago de Compostela, Spain, (1995).

[8] http://epims.gsfc.nasa.gov/og/index.cgi

[9] M. Capeans, Nucl. Instr. and Meth. A 446 (2000) 317.

[10] T. Zeuner, Nucl. Instr. and Meth. A 446 (2000) 324.

[11] F. Guarino et al., ATL-INDET-99-011 (1999).

[12] S. Bachmann et al., Proc. of IEEEE Nucl. Sci. Symposium, (2000).

[13] R. Bouclier et al., Nucl. Instr. and Meth. A 350 (1994) 464.

[14] F. Guarino, ATL-IT-FN-0002 (1998)

[15] P. Chiggato, EST/SM/DA Report (2001).

[16] S. Kappler, Proc. Int. Workshop on Aging Phenomena in Gaseous Detectors, 2001.

[17] A. Romaniouk, Proc. Int. Workshop on Aging Phenomena in Gaseous Detectors, 2001.

[18] M. Bosteels CERN/ST/CV, Private communication.

[19] L.M. Yeddanapalli, Journal of Chem. Phys. Vol 10, No 5 (1942) 249.

[20] DW. Hess, Plasma Chemistry in Wire Coating, Proc. of the Workshop on Radiation Damage to Wire Chambers, LBL-21170 (1986), 15.

[21] R. Bouclier et al.., Nucl. Instr. and Meth. A 346 (1994) 114.

[22] J.Wise, Ph.D. Thesis, LBL-32500 UC-414 (1992).

[23] J. Va'vra, Proc. Procedings of the Workshop on Radiation Damage to Wire Chambers, LBL-21170 (1986). 